\title{
Development and accuracy determination of a two-component Doppler Global Velocimeter (DGV)
}

\author{
Stephen M. Naylor \\ West Virginia University
}

Follow this and additional works at: https://researchrepository.wvu.edu/etd

\footnotetext{
Recommended Citation

Naylor, Stephen M., "Development and accuracy determination of a two-component Doppler Global Velocimeter (DGV)" (1998). Graduate Theses, Dissertations, and Problem Reports. 3142. https://researchrepository.wvu.edu/etd/3142

This Dissertation is protected by copyright and/or related rights. It has been brought to you by the The Research Repository @ WVU with permission from the rights-holder(s). You are free to use this Dissertation in any way that is permitted by the copyright and related rights legislation that applies to your use. For other uses you must obtain permission from the rights-holder(s) directly, unless additional rights are indicated by a Creative Commons license in the record and/ or on the work itself. This Dissertation has been accepted for inclusion in WVU Graduate Theses, Dissertations, and Problem Reports collection by an authorized administrator of The Research Repository @ WVU. For more information, please contact researchrepository@mail.wvu.edu.
} 


\title{
Development and Accuracy Determination of a Two-Component Doppler Global Velocimeter (DGV)
}

\author{
Dissertation
}

Submitted to the College of Engineering and Mineral Resources

West Virginia University

Morgantown, WV

In Partial Fulfillment of the Requirements

for the degree of

Doctor of Philosophy

By

Stephen M. Naylor

July 1998 


\section{TABLE OF CONTENTS}

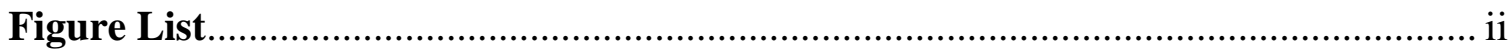

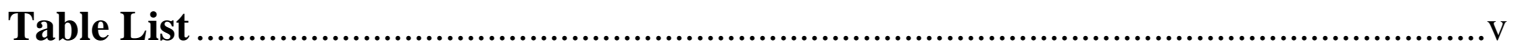

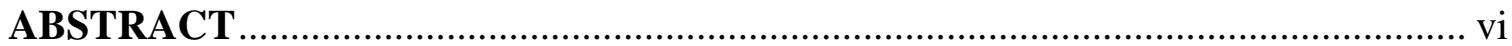

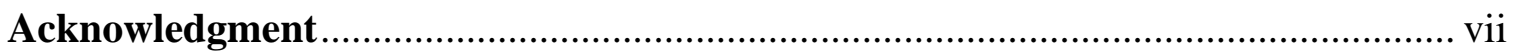

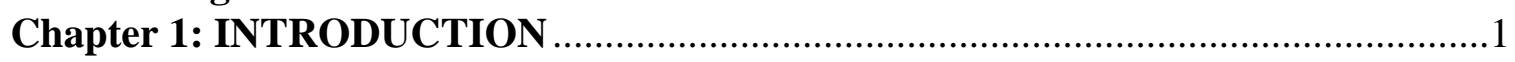

Chapter 2: PREVIOUS WORK IN DOPPLER VELOCIMETRY ………................

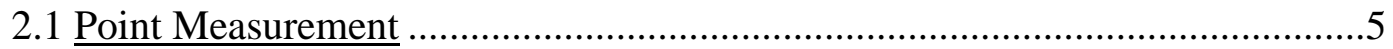

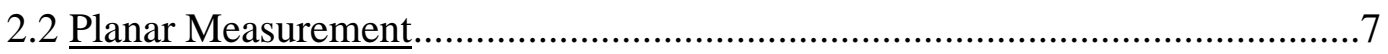

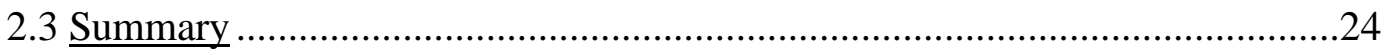

Chapter 3: APPARATUS AND CONFIGURATION ……….................................2

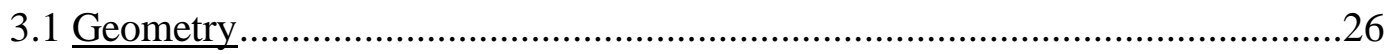

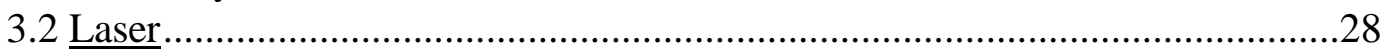

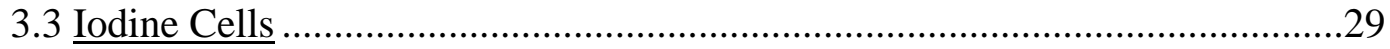

3.4 Reference System.....................................................................................

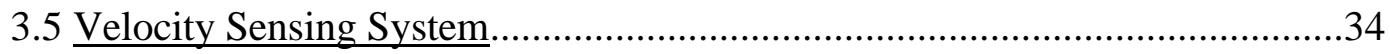

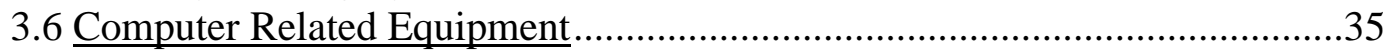

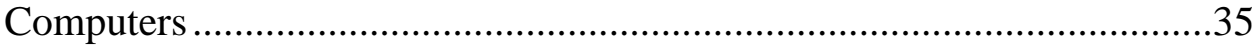

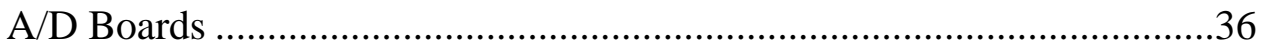

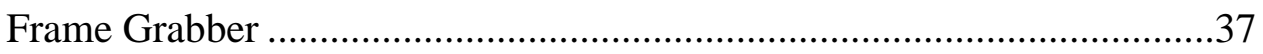

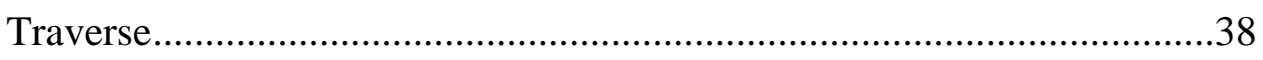

Chapter 4: DATA ACQUISITION AND IMAGE PROCESSING .............................39

4.1 Synchronization .........................................................................................

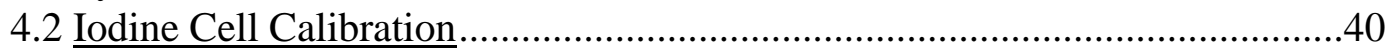

4.3 Image Processing Procedure …………………….......................................44

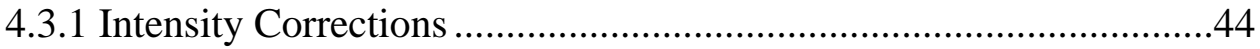

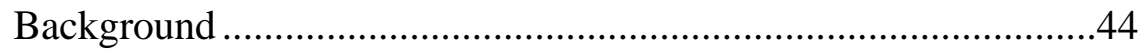

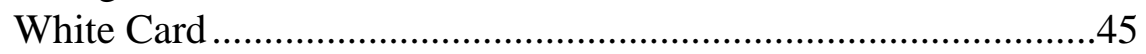

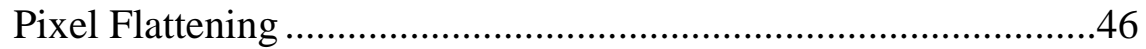

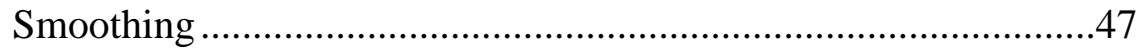

4.3.2 Spatial Correction-Dewarping ………………………...................49

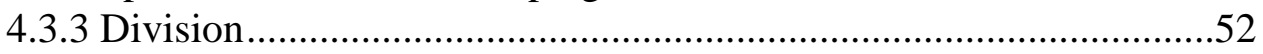

Chapter 5: VELOCITY MEASUREMENT EXPERIMENTS ……………............54

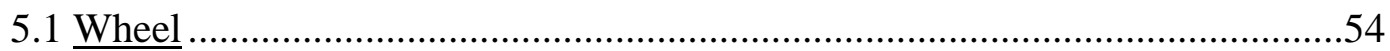

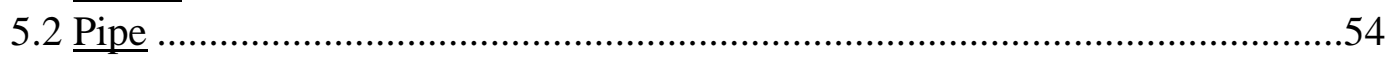

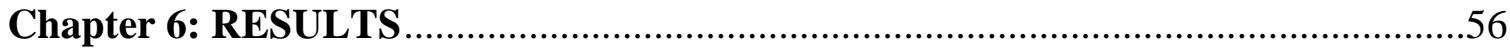

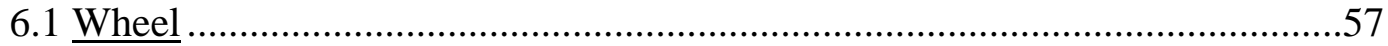

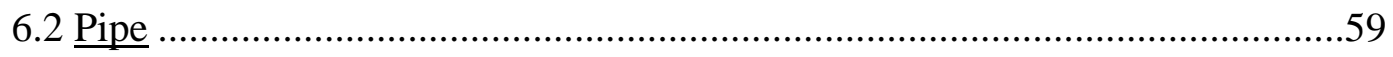

Chapter 7: CONCLUSIONS and RECOMMENDATIONS ………………….........65

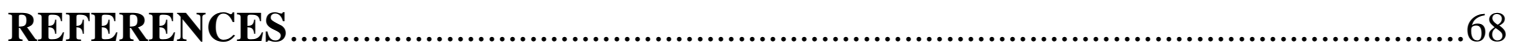

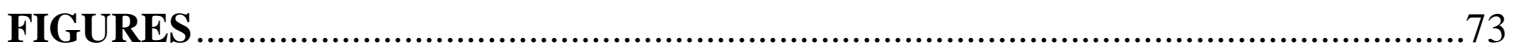

APPENDIX A--Discussion of Specially Written Software ...........................................103

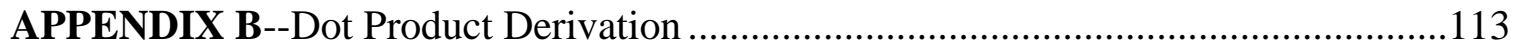

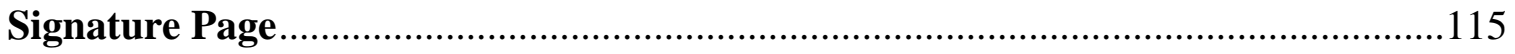




\section{$\underline{\text { Figure List }}$}

Figure 3.1. Top view of a DGV velocity measuring component

Figure 3.2. Vector geometry for a 2-compontent DGV system.

Figure 3.3. Top view schematic of system setup for rotating wheel experiment

Figure 3.4. Drawing of experimental setup for measurement of turbulent pipe flow

Figure 3.5. Photo of Iodine cell with copper jacket and thermal ground visible

Figure 3.6. Response of temperature controller on $\mathrm{I}_{2}$ cell stem and body temperatures. Controller was activated at Time $=2$ minutes

Figure 3.7. Temperature variation of an $\mathrm{I}_{2}$ cell stem showing correlation with ambient temperature

Figure 3.8. Calibration curve fits from all three I2 cells showing slope inconsistencies

Figure 3.9. Diagram of reference system components

Figure 3.10. Ratio of the voltage outputs of the reference system photodiodes over the time span that an image is acquired $(1 / 60$ sec. $=833$ samples $\div 50 \mathrm{kHz})$.

Figure 3.11. Comparison of noise levels from the 16-bit A/D boards when inputs being read are grounded

Figure 3.12. Drawing of computer controlled traverse with pipe flow apparatus installed

Figure 4.1. Logic to synchronize the frame grabber with the A/D board residing in a separate computer

Figure 4.2. Single calibration curve from reference photodetector system showing mode spacing .79

Figure 4.3. Photodetector voltage ratio showing same-mode frequency variations.....80

Figure 4.4. Typical calibration data and curve fits for all three iodine cells . .81

Figure 4.5. Plot of the curve fits for 2 calibrations for cell \#1 with an enlarged view showing variation in slope

Figure 4.6. Plot of the curve fits for 2 calibrations for cell \#2 with an enlarged view showing variation in slope 
Figure 4.7. Block diagram of image processing steps. Steps 1-5 are performed on each image, while in steps 6-10, signal and reference images are combined to generate a velocity image

Figure 4.8. Horizontal and vertical cuts through a flatfield image before (top) and after (bottom) applying a pixel sensitivity correction

Figure 4.9. Visualization of camera/lens MTF

Figure 4.10. Diagram and equations used in dewarping of data images

Figure 4.11. Overlay of signal and reference dewarped dot card images showing alignment for both Component \#1 (Top) and Component \#2

(Bottom)

Figure 6.1. Colorized DGV Component \#1 average velocity image of rotating wheel

Figure 6.2. Colorized DGV Component \#2 average velocity image of rotating wheel.....

Figure 6.3. Cuts of the average DGV velocity measurement from Component 1 of a rotating wheel surface, reduced in direction of motion

Figure 6.4. Cuts of the average DGV velocity measurement from Component 2 of a rotating wheel surface, reduced in direction of motion

Figure 6.5. Colorized 2-component DGV tangential average velocity image of rotating wheel

Figure 6.6. Colorized 2-component DGV normal average velocity image of rotating wheel.

Figure 6.7. Cuts of the two-component, average orthogonal velocity measurement parallel to the surface of a rotating wheel

Figure 6.8. Cuts of the two-component, average orthogonal velocity measurement normal to the surface of a rotating wheel

Figure 6.9. Figure 6.6 reduced without the white card correction

Figure 6.10. Cuts through image in Figure 6.9 showing the need for white card correction

Figure 6.11. DGV averaged velocity image of fully turbulent pipe flow (Component \#2)

Figure 6.12. DGV averaged velocity image of turbulent jet flow, 1 diameter from exit (Component \#2) ..... 
Figure 6.13. Cuts through Figure 6.11, Component \#2 DGV fully turbulent pipe flow velocity measurements

Figure 6.14. Cuts through Figure 6.12, Component \#2 DGV jet velocity measurements 1 diameter from the exit

Figure 6.15. DGV averaged velocity image of turbulent jet flow, 1 diameter from exit (Component \#1 - run 2)

Figure 6.16. DGV averaged velocity image of turbulent jet flow, 1 diameter from exit (Component \#2 - run 2)

Figure 6.17. Cuts through Figure 6.15, Component \#1 DGV jet velocity measurements 1 diameter from the exit

Figure 6.18. Cuts through Figure 6.16, Component \#2 DGV jet velocity measurements 1 diameter from the exit

Figure 6.19. DGV averaged velocity image of turbulent jet flow, 2 diameters from exit(Component \#1)

Figure 6.20. DGV averaged velocity image of turbulent jet flow, 2 diameters from exit (Component \#2) .....

Figure 6.21. Cuts through Figure 6.19, Component \#1 DGV jet velocity measurements 2 diameters from the exit

Figure 6.22. Cuts through Figure 6.20, Component \#2 DGV jet velocity measurements 2 diameters from the exit

Figure 6.23. DGV averaged velocity image of a turbulent jet, 4 diameters from the exit (Component \#2)

Figure 6.24. Cuts through Figure 6.23, Component \#2 DGV jet velocity measurements 4 diameters from the exit

Figure 6.25. DGV velocity image of turbulent jet flow, 6 diameters from exit (Component \#1)

Figure 6.26. DGV velocity image of turbulent jet flow, 6 diameters from exit (Component \#2) .98

Figure 6.27. Cuts through Figure 6.25, Component \#1 DGV jet velocity measurements 6 diameters from the exit

Figure 6.28. Cuts through Figure 6.26, Component \#2 DGV jet velocity measurements 6 diameters from the exit

Figure 6.29. Cuts through Figure 6.11, Component \#2 DGV fully turbulent pipe flow velocity measurements with velocity offset set to match pitot probe data

Figure 6.30. Cuts through Figure 6.12, Component \#2 DGV jet velocity measurements 1 diameter from the exit with velocity offset set to match pitot probe data 
Figure 6.31. RMS fluctuations of a turbulent jet, 6 diameters from the exit (Component \#1)

Figure 6.32. RMS fluctuations divided by the local mean of a turbulent jet, 6 diameters from the exit (Component \#2).

Figure 6.33. Cuts through Figure 6.25, RMS fluctuations of a turbulent jet

Figure 6.34. Cuts through Figure 6.26, RMS fluctuations divided by the local mean velocity 4 diameters downstream of a turbulent jet

\section{$\underline{\text { Table List }}$}

Table 1. Standard deviations of dot centroids (in pixels)

Table 2. Standard deviations $(\sigma)$ and measurement accuracies

of average image $\mathrm{X}$ and $\mathrm{Y}$ cuts $(\mathrm{m} / \mathrm{s})$ for components 1 and 2

Table 3. Standard deviations $(\sigma)$ of orthogonal velocity components, and tangential component measurement accuracies $(\mathrm{m} / \mathrm{s})$. .58 


\begin{abstract}
A two-component Doppler Global Velocimeter (DGV) system was constructed and tested to research problems associated with the accuracy of this unique system. The uniqueness of the system lies in its ability to simultaneously and non-intrusively measure velocities in a laser illuminated plane. A key component of the system is a frequency discriminating optical filter containing iodine vapor which allows direct measurement of the Doppler frequency shift caused by particle motion. Corrections for optical distortions and non-uniform intensities as well as the conversions from intensity data to velocity data are performed by an extensive image processing algorithm. Measurements were made of a 12" diameter rotating wheel and turbulent pipe/jet flow. Both RMS deviations and velocity range measurement errors from a single component for the rotating wheel with a maximum velocity of $58 \mathrm{~m} / \mathrm{s}$ were less than $2 \%$, better than most published results, to date, for similar systems. Pipe/jet flow profiles agreed very well with the shape of pitot probe measurements. RMS errors were on the order of 5-10\%, but velocity offset error was as much as $10-15 \%$ of the $42 \mathrm{~m} / \mathrm{s}$ velocity range. DGV measured turbulence intensities at the center of the pipe, 4 diameters downstream agreed with hot wire data, with some reservations. Several factors such as repeatability of calibrations, precision of wheel/pipe speed measurement, measurement of viewing angles, and 8-bit camera digitization contributed to the errors in DGV velocity data. Proper techniques for preparing and acquiring correction images are also critical steps toward the goal of producing accurate velocity data.
\end{abstract}




\section{$\underline{\text { Acknowledgment }}$}

This work was funded by AFOSR Grant F49620-94-1-0434, Drs. James M. McMichael and Mark Glauser, technical monitors. Special thanks to John "Tex" Smith for acquiring and recording all pitot probe data. 


\section{Chapter 1: INTRODUCTION}

In any analysis of fluid flow, the ability to measure the velocities in the flow is a basic requirement on the way to basic understanding and modeling. The types and techniques of these measurements are varied. They can be broken down into two classes: intrusive and non-intrusive; non-intrusive methods being principally optically based.

Intrusive velocity measurements involve placing a physical object (or probe) into the flow at the point of measurement. Point-wise measurement techniques of this type include hot wire and hot film anemometry, as well as pressure sensing probes (pitot and pitot-static).

The hot wire and film probes operate on the principles of convective heat transfer and electrical resistive heating (Bruun, 1995). A small, thin, wire is mounted on two probe supports, and a voltage is applied through the activation of a wheatstone bridge. The probe takes the place of one of the resistors in the bridge, so that a small resistance change corresponds to a large voltage change. When a voltage is applied, the wire then heats and is cooled by convection to the cooler fluid passing around it. A change in the temperature of the wire corresponds to a change in resistance, which can then be measured with a resistance bridge. The addition of a feedback control system produces a more useful system in most cases by varying the bridge current to produce a constant resistance through the wire, and therefore a constant temperature. When set-up in constant temperature mode, a hot wire probe can deliver continuous velocity data with a frequency response in the tens of thousands of hertz, which is very useful in measuring turbulent quantities.

The pressure probes either measure total (stagnation) pressure at the probe face and static pressure at the wall at the same streamwise location, or have the total and static pressure ports on the head of the probe (pitot-static probe). The pressure probes have a maximum frequency response in the tens of hertz, so they are mainly used for measuring mean flow quantities (Rae and Pope, 1984).

Advantages of the hot wire and pressure sensing probes include their low-cost, fast, easy implementation at a point in the flow where the sensing position is known immediately. The main disadvantage is that the probe body itself disturbs the flow at the 
measuring point and thereby decreases the accuracy of the measurement. Also, these are one-dimensional devices; that is, to resolve the full three-dimensional velocity vector, three of these devices must be placed at the same or nearly the same point, oriented at different angles. For a hot wire probe, an additional difficulty lies in distinguishing the direction of the velocity since the wire responds to all velocity vectors aligned perpendicular to the span of the wire with nearly equal sensitivity. Minimization of this problem requires careful design of the experiment and/or the addition of more probes.

Extending the point probes into line or area measurement devices is just a matter of arranging several probes into a row and is done mainly with pitot probes to form a "wake rake", which is an instrument commonly used in momentum deficit wake surveys. The pressures from the line of probes can be read to determine a velocity profile, which is crucial to momentum equation integrations.

Optically based measurement instruments usually use a laser beam as the source of the interrogating light. Fluid molecules themselves provide very weak scattering; consequently, the flow to be measured generally needs to be seeded. The degree to which the particles follow the actual flow varies depending on the size of the seeding and the character of the flow. For a given flow, smaller particles follow the flow better, but provide less scattered light in return. Hence, there is a trade-off between particle size and scattered light intensity. Point-based methods utilize laser light for its coherent, collimated beams to provide precise spatial location and predictable interference patterns. Laser-2-focus (L2F) is a method which focuses two laser beams very near each other in the flow while receiving optics record the two flashes that come from a particle passing through the beams. The time between the flashes determines the velocity of the particle since the spacing of the beams is known. Care must be taken, however, to ensure that the same particle passes through both foci. Laser velocimetry (LV) relies more heavily on the coherent property of lasers to create interference patterns when the beams are intersected. Then, when a particle passes through the intersection point, it scatters the light and dark bands of the interference fringes at a frequency proportional to its velocity. Again, both of these point-based measurement techniques are one-dimensional. By far the most popular, LV has seen great success as a three-component system by intersecting 
three pairs of beams of different frequencies from different directions at a single point.

Planar optical velocimetry systems have only recently matured enough to provide accurate test data. The most prominent of these systems is Particle Image Velocimetry (PIV). Normally, PIV works by opening the shutter on a CCD array, then illuminating the measurement plane with two pulses of laser light spread into a two-dimensional sheet. The pulses are timed such that a small but measurable amount of particle travel can be observed in the resulting image. The path of the individual particles can be resolved either by computer or by eye, but either way, the velocity is determined based on the time between pulses and the path length of the particle. The advantages include low cost/complexity and ease of implementation as well as a good qualitative feel for the velocity data. The disadvantages lie primarily in the data reduction scheme. The examination of frame after frame of images, tracking individual particles by hand, is tedious at best. Assigning a computer the task involves lengthy correlation routines which are needed to increase the chance that the same particle is being tracked from frame to frame, and that particles that entered or left the volume of the laser sheet are not mistakenly used in velocity calculations. The tradeoff lies between long computing times and inaccurate data. This method provides two-dimensional information, but only in the plane of the laser sheet. To extract a third component, a difficult and not yet perfected method involving a laser hologram defined volume where particles are tracked in three dimensions needs to be implemented.

The measurement technique which is the focus of this work falls in the more recent category of optical filter based, planar, velocity measurement systems. In this category, the scattered light collected from the particles in the flow is filtered with some frequency discriminating device such that a Doppler shift in the light frequency produces a change in intensity through the filter. However, variations in the scattered light intensity would also be interpreted as Doppler shifts, necessitating the use of an unfiltered or reference image which is used to remove the sensitivity to scattered light intensity. The ratio of the filtered and unfiltered images is then proportional to velocity according to the characteristics of the optical filter. One advantage of a planar frequency discriminator is its relative insensitivity to particle size and seeding density. There are still size and 
concentration issues to contend with, but the technique is not concerned with the individual particles as the other methods are. Velocity can be measured perpendicular to the light sheet just as well as any other direction. Discrete particle independence allows velocity measurements to be found in the plane of the laser sheet at all locations providing scattering, and also gives the opportunity to increase spatial resolution.

The focus of the research presented here was to construct and analyze the accuracy of a two-component, Doppler sensitive, planar optical velocimeter. The first step was to develop a two-component point system based on the same methods as the planar system. The point system allowed familiarization of the theory and configuration of the measurement system without the burden of an extensive data reduction process. The next step was to switch the light intensity sensing devices from point-oriented photodetectors to plane-oriented CCD arrays. The planar system was then tested by measuring the velocity of a rotating wheel, as well as fully turbulent pipe/jet flow. The rotating wheel uniformly scatters laser light, has a homogeneous surface, and has a continuous variation of velocity that includes zero. Therefore, the rotating wheel is a device which has fewer variables to consider when identifying sources of error. However, the usefulness and advantages of the DGV measurement system are best demonstrated in a complex, turbulent fluid flow such as pipe flow, or, when measured several diameters downstream of the pipe exit, low-speed jet flow.

A side by side comparison of DGV velocity measurements with measurements from another, more established instrument has been largely absent in the field of DGV research. This work documents, in detail, the results of such a comparison. Research in this field can only benefit from an experimental investigation into the accuracy of this DGV system when stood next to familiar and established standards. 


\section{Chapter 2: PREVIOUS WORK IN DOPPLER VELOCIMETRY}

\section{$\underline{2.1 \text { Point Measurement }}$}

As a point system, a Doppler sensitive, filtered velocimeter does not bring much new to the family of point measurement methods. Perhaps this is why many of the researchers currently developing this technology start with a true planar system imaged by CCD arrays. However, three researchers have investigated such a point system either as a stepping stone to a more unique planar measurement technique or to develop it as an additional point system on its own merits. Unlike LV, a filter based point system provides a continuous analog signal with which to calculate time dependent characteristics of the flow. Hoffenberg (1993) used a continuous wave (cw) laser to illuminate smoke at the exit of an axisymmetric jet, and an iodine vapor filled cell as the optical frequency filter. A single component point system was constructed using two photomultiplier tubes (PMTs) as the intensity measuring devices for both the filtered and unfiltered light paths. Two measures were taken to ensure that light from the same probe volume was being seen by both PMTs. First, light collected by a main frontal lens was passed through a beamsplitter where half of the light went straight to the reference PMT and the other half was filtered. The other measure was the addition of pinholes mounted on $\mathrm{X}-\mathrm{Z}$ traverses just in front of the PMTs, presumably at the focal point of the front lens. The pinholes were adjusted until the cross correlation of the two signals was at a maximum.

Roehle and Schodl (1994) also used spatial filtering, but placed a single pinhole at the focal point of the front lens in front of the beamsplitter. In this configuration, no examination of the cross correlation is necessary, as the PMTs are receiving light from the exact same point. The remainder of Roehle's setup included a cw argon-ion laser and an iodine cell as the optical filter for taking measurements in a jet flow. The cw argon laser is effectively pulsed via an optical chopper, then the photodetectors (one filtered) are amplified in phase with the laser pulses with a lock-in amplifier. The signals are then sent to an analog divider to remove the effects of varying brightness and sampled with an $\mathrm{A} / \mathrm{D}$ converter.

Filtering scattered light received from Doppler shifts works well if the laser 
frequency remains constant. Otherwise, a laser drift would be construed as a velocity change. There are two ways of dealing with this possibility; either to measure the drift and correct for it, or to control the frequency of the laser to assure that there is no drift. The latter is the configuration that Roehle and Schodl use. Their cw argon laser is fitted with a piezo-electric translator on the back laser mirror so that the effective cavity length, and hence, the frequency can be kept constant. The reference signal for their controller comes from monitoring iodine hyperfine lines which are a substructure within the absorption "well". The hyperfine lines of iodine are much less dependent on temperature and pressure than the main absorption well, so they serve well as a frequency reference.

Measuring and subtracting the laser frequency is a more common and less complex way of dealing with laser drift. Hoffenberg described a reference channel assembly just for that purpose as a future improvement in his system. The system he describes is common to other researchers during and since the time that the paper was published (1993). The outlined reference system was similar to a velocity sensing setup, but instead of passing scattered light through an optical filter, a portion of the laser beam was fed directly to the beamsplitter, and then to two photodetectors. One beam path passed through an iodine cell, and the ratio of the filtered to unfiltered signals gave a relative measure of the laser frequency. Subtracting this from the scattered light frequency left a measure of true Doppler shift.

McKenzie (1995) used a point system to compare readings made on a rotating wheel where the velocity is known at all points. Although the principle is the same, the setup used was very different. A pulsed, injection seeded, frequency doubled Nd:YAG laser was used in place of a cw argon laser, and only one pair of photodetectors was used for both signal and reference systems. Two signals which occur simultaneously at the source were received separately by the photodetectors by time delaying one signal. The scattered light was sent to the photodetectors via a fiber optic cable which was more than ten times longer than the fiber carrying the laser reference signal. Since the pulse length was very short (on the order of 20ns), the signals were effectively separated. The signals were sent from the diodes to a $10 \mathrm{~ns}$ integrator which then gave 12-bit digital outputs proportional to the energy of each reference and signal pulse. 
Initially, Hoffenberg used no laser frequency drift compensation (Hoffenberg and Sullivan, 1993), and the results showed the effects of this. When compared to LV data or theory, the point Doppler measurements of axial jet velocities less than $100 \mathrm{~m} / \mathrm{s}$ were up to $25 \%$ in error. It was recognized that drift was the primary source of error, and future configurations were suggested which would include some type of compensation, either monitoring or control. Roehle controlled the laser frequency and achieved stability of \pm 1 MHz. With drift essentially removed, an accuracy of $\pm 3 \mathrm{~m} / \mathrm{s}$ in a jet flow between 40 and $130 \mathrm{~m} / \mathrm{s}$ was achieved, which translates to a maximum error of $7.5 \%$. These accuracies were obtained by using L2F as the standard measurement, but since that method has inaccuracies of its own, the margin of error could be slightly different. Compensation for drift by monitoring is equally effective, according to results obtained by McKenzie. When measuring velocities on a rotating wheel, the velocity is more precisely known, which makes McKenzie's $\pm 2 \mathrm{~m} / \mathrm{s}$ maximum error all the more impressive. This error is on the order of the 12-bit A/D board's discretization error of \pm 1 bit.

\subsection{Planar Measurement}

The idea of using an iodine filled cell as a frequency dependent optical filter was patented by H. Komine working at the Northrop Corporation in 1990. He called it a Doppler Global Velocimeter, or DGV. This patent is the basis for several papers with topics on filtered particle scattering (FPS), and arguably those based on a slightly different method called filtered Rayleigh scattering (FRS). The patent describes a basic planar FPS velocity measuring system containing a frequency controlled laser projected into a sheet that intersects a seeded flow, with the receiving optics gathering the scattered light. The receiving optics package first splits the incoming scattered light, then passes half through a molecular iodine cell to a CCD array, and the other half straight to a second CCD array. The setup is very similar to the point systems described above; it ought to be, since the point systems were motivated by this same planar idea, and exist largely as a simplification to the complexities of obtaining an accurate planar velocity measurement. Much of the research that followed the patent included small changes and improvements in the configuration and signal processing of the system. For example, the 
patent calls for routing the images from the CCD cameras to an analog video divider, then storage on a high-quality video cassette recorder. Researchers have abandoned that technique because of problems in cleanly separating individual signals into channels on a VCR. Also, the advancement of high speed digital frame grabbers to acquire the signals, digital video image processing to handle the division, and personal computers for storage and display of velocity information makes the digital path much more appealing.

Komine and Brosnan (1991) and Komine, et al. (1991), in proof of concept papers, expand on the idea of the original patent by using both a CW and a pulsed laser for illumination, and video frame grabbers for image acquisition. Still present though, is the analog normalization, thought by many other researchers to be a significant source of error since small errors in the optics prohibit exact reference-to-signal camera alignment. Current thinking is that this division can be more accurately carried out after image processing software has stretched and shifted the images so that they overlay more precisely. In the $\mathrm{CW}$ laser experiment, no measures were taken to account for the laser drift, while the pulsed laser was controlled by a feedback loop with correction signals coming from a spectrum analyzer. Velocity data were generated in simple flows from these experiments, but no comparison was made with velocity readings from any other measurement technique. Therefore, the accuracy of this preliminary system was undocumented.

Ford and Tatam (1995) describe a basic, single component system roughly based on the patent by Komine, except the system includes digital image acquisition and processing components. Results from the measurement of the velocity of a rotating wheel were presented. The authors made a detailed examination of the effect of the angular variation across the viewing area, both due to the scattering angle deviation away from the center of the image, and the incident laser angle resulting from spreading the beam through cylindrical optics. Calculations showed that the error due to angular variation across the field of view is on the order of the resolution of the system when the angle reaches 5 degrees on either side of center, and 10 degrees of incident beam angle.

A more complex data processing scheme is at the heart of a three-component DGV system in operation at NASA Langley under the supervision of J. Meyers. In his 
introductory paper, co-written with Komine (1991), Meyers essentially reiterates the Komine patent and presents details on the signal processing along with laboratory experimentation and preliminary wind tunnel testing. According to Meyers and Komine, there are three main responsibilities of the signal processing scheme: to synchronize the reference and signal cameras, to overlay and normalize the images, and to correct for varying pixel sensitivities across the CCD array. To synchronize the CCD cameras, the internal sync pulses were disabled and an externally generated pulse was sent to all of the cameras and associated frame grabbers, thus assuring that all images were simultaneously sampled. Normalization of the images can be done either with an analog divider, or digitally, using frame grabbers. The accuracy of either method depends on the pixel-topixel alignment of the images from the signal and reference cameras. Optimally, a beamsplitter will split the image undistorted so that the adjustment of pathlength, pan, and tilt on the cameras is enough to assure that each pixel in the signal camera receives light from the same volume as does a corresponding pixel in the reference camera. This was assumed to be the case in the preliminary system. For real-time data acquisition, it is necessary to divide these two images with an analog divider, then sample or record the resultant image. A much more accurate, albeit significantly slower, method involves simultaneously sampling each raw image, then digitally dividing pixel by pixel to produce the normalized image. The analog divider can still be used in parallel, and its results sent to a monitor, for a visual check of the status and integrity of the data. The correction for CCD pixel sensitivities is done on a camera-by-camera basis to account for manufacturing inconsistencies.

DGV data was taken by Meyers, et al. (1991) on a rotating wheel, a subsonic jet, and $75^{\circ}$ delta wing in a wind tunnel. Qualitatively, the results were as they should be, and agreed with known velocities. However, there was still no direct quantitative comparison with previous or concurrently measured data. The unprocessed results were very noisy, mainly as a result of noisy CCD detectors. To smooth the data, several images were averaged yielding mean velocities. If no meaningful data can be deduced from individual frames, real-time data acquisition is an unrealistic goal. The results from these experiments and both the analog and digital approach to the signal processing are 
presented in more detail in a follow-up paper by Meyers, Lee, and Cavone (1991).

As work on this system progressed, Meyers $(1992,1994)$ added more signal processing steps to the data reduction scheme for the images acquired by the video cameras. One realization arising from the wind tunnel experiments was the need for exact pixel overlay which could not be achieved by simply moving the cameras. The optics used to steer the image to the cameras, as well as the pixel spacing in the cameras themselves, contained imperfections that made it impossible to align all the pixels in any one image pair. The image processing algorithms were tasked with the image warping needed to minimize these imperfections. After the warping algorithms were applied, spatial cross correlation routines were used to correct any remaining misalignment during data taking by identifying the proper pixel position at the peak value of the correlation. The pixels were then shifted to that position, providing maximum pixel to pixel correlation for the entire image. Before the images are ratioed, background light images recorded under the same conditions but with no seed particles are subtracted from each image. Then, the correction for unequal pixel sensitivities is performed. The images were then lowpass filtered to remove some of the CCD camera noise, just prior to being normalized. Additional light-intensity problems were addressed as a result of images recorded which were saturated in the center of the light sheet, and very dim near the edges. The cause was traced to the way the light sheet was created, by simply expanding the laser beam through lenses. When a sheet is formed in this manner, the original Gaussian intensity distribution in the beam is evident in the sheet, producing high intensity at the center and lower intensity on the sides. The fix for this had to be in the hardware configuration since the cameras were not capturing useful images. A high speed galvanometric scanner was used to 'fan' the beam into a sheet of uniform intensity. Additional concerns about the variation in the laser propagation angle, which is vital to determining velocity, are unwarranted if the spreading angle of the laser sheet is kept small.

The need for all of the additional signal processing was identified through problems in accuracy encountered in the laboratory and wind tunnel testing. One result of additional processing is the abandonment of the analog method of data reduction. While 
fast, the analog method does not provide an acceptable level of accuracy to allow the DGV system to evolve as a formidable planar velocity instrument. In an application oriented paper (Gorton, et. al., 1996), Meyers uses the DGV system in place at NASA Langley to measure aerodynamic rotor-tail-fuselage interaction on a small scale helicopter model. The results were compared with LV measurements taken in the same locations, and significant errors in the DGV data were apparent. The main source of these errors was found to be large temperature fluctuations in each of the iodine cells used for frequency discrimination. Insulation was added around the filters, and corrections were applied in post-processing in an attempt to minimize the effects of these fluctuations, but they still caused an increase of $5 \mathrm{ft} / \mathrm{s}$ in the velocity uncertainty. Because of this test, an insulating box surrounding the iodine cell was constructed to isolate it from high convection situations. Even with that precaution, the barrel and sidearm temperatures were measured at each data run to calculate a theoretical absorption curve with which to calculate velocities. The next generation DGV system at NASA Langley has more improvements to improve the accuracy of the system. The most significant contributor to those changes is the incorporation of a pulsed laser. This will continue and improve upon the research of Komine and McKenzie. Also, 10-bit Matrox frame grabbers replaced the 8-bit frame grabbers to provide better velocity resolution and, less cross-talk between cameras. Future plans may include incorporating sealed cells which contain no iodine crystals, only vapor. With no crystals present, the vapor pressure, and therefore the absorption characteristics of the cell are much less likely to vary with small changes in temperature.

Beutner and Baust (1997) describe a test on a delta wing model that took place in the Subsonic Aerodynamic Research Laboratory at Wright Laboratory using essentially the same system as Meyers at NASA Langley. A cw laser was fanned into a light sheet and placed perpendicular to the flow at various chord locations on the model. Due to the size of the test and the distances involved, the three-component system did not use a beamsplitter system on each component to ensure angular invariance between the reference and signal images. Instead, the cameras were placed close to one another and focused independently on the same region of the flow. This small angular difference 
could have had consequences not accounted for in the data processing such as intensity variation versus viewing angle due to Mie scattering. In what Beutner describes as a "velocity discriminating flow visualization technique," measurement results for the velocity distribution of a very complex vortical flow are presented in very general terms only.

Also working at NASA Langley, but developing a system independent of Meyers and his researchers, are Smith and Northam (1995, 1996). Smith and Northam also illuminate a seeded flow, but use a frequency controlled pulsed laser to produce the light sheet, and a single camera to image both signal and reference images. The single camera feature allows the use of higher quality, more expensive cameras, or allows more velocity components to be implemented since cameras are a major cost item. Another advantage is the reduction of some of the image processing relating to unequal camera pixel response between reference and signal cameras. Since both images are placed side by side on the same CCD array, errors due to manufacturing inconsistencies should be less than they would be between two different cameras. Different camera and lens configurations were studied, all by measuring supersonic and sonic jet flow. In the first experiment, a liquid cooled high performance camera was used to acquire data. The camera required about 10 seconds to acquire and transfer an image, so data acquisition at the maximum laser pulse repeat rate of $30 \mathrm{~Hz}$ was impossible. Mean velocity images were acquired by illuminating the measurement area with several laser pulses, and single shot images were gathered by shuttering the camera and exposing it to one pulse. In the second experiment, a less expensive, non-intensified Sony camera was used. This camera had no problem gathering data at 30 frames per second, but image brightness was considerably lessened. Signal strength was reduced to the extent that the flow had to be slowed to sonic speed, so that the concentration of seed particles could be increased. In the last experiment, a larger diameter lens was fitted to the Sony camera. The primary reason for this was to reduce noise from laser speckle. Smith and Northam (1995) derive an equation for determining the uncertainty that laser speckle adds to a measurement image. The effect of speckle on a measurement is a function of several factors including a direct proportionality with the magnification of the image and the f number of the lens. 
These two parameters are more easily changed than the other factors such as wavelength, and a smaller f-number has the additional advantage of collecting more light, which permitted the flow velocity to be returned to supersonic with the data collection rate unchanged at $30 \mathrm{~Hz}$.

In a subsequent paper, Smith (Jan, 1998) investigated a compressible jet with a single component, pulsed laser DGV system. The flow had a novel core/co-flow seeding apparatus which enabled velocity measurements throughout the mixing layer of the jet. In addition, the seeding could be enabled for both the co-flow and the core separately. Results of the mean core velocity showed good agreement with both isentropic calculations based on the exit conditions and pitot probe data. The fluctuations both in the shear layer and in the core were measured to be slightly higher than the comparison data. The causes of this discrepancy were identified as seed laser dither and speckle noise. Great care was taken in this paper to minimize the velocity deviations caused by laser speckle. In a separate but related effort, Smith (June, 1998) has researched the problem of reducing speckle noise for DGV systems utilizing pulsed lasers, and applied his findings in this paper. Laser light scattered by both a moving and stationary screen provided reference and signal images Smith could ratio to remove the RMS variation due to surface roughness or beam intensity variation, yielding variation in brightness due exclusively to laser speckle. Methods were then studied in an attempt to minimize this variation.

The primary noise reduction mechanism found was to influence the size and concentration of speckle "dots" on the CCD array. This was done several different ways, with varying degrees of success. One of the most effective methods was to decrease the f-number of the lenses used to collect the scattered laser light. Reiterating the derivation presented in his previous paper, Smith found a direct inverse proportionality between the lens f-number and the signal to noise ratio of speckle induced images. Another component that had a strong influence on the speckle noise is the type of CCD array used in the camera. Both pixel spacing and readout method are factors, with a more compact (higher fill factor), frame transfer array favored. Less effective was a double pulse exposure with the second pulse delayed by approximately $20 \mathrm{~ns}$ meant to allow the flow to 
slightly change the scattering characteristics as seen by the imaging system. However, 20ns was not enough time to allow the flow to move, and therefore the reference and signal exposures were still highly correlated.

McKenzie (1996) also used a pulsed laser and a single camera for each velocity component in his setup. He shows that a high quality camera makes a significant difference in accuracy, and therefore it is more cost effective to use one high quality camera than to use two average quality cameras. Also, it was shown that the scientific grade, cooled, slow-scan CCD arrays had less noise than many other types of photo sensitive devices, including photomultiplier tubes and photodiodes. In addition to virtually eliminating CCD camera noise by selecting a high quality scientific grade camera, McKenzie sought to compensate for the small pulsed laser frequency drift. Laser drift contributes both random, shot to shot noise, and systematic, long term error. By monitoring the laser frequency separately and subtracting any variation from the measured Doppler shift, the impact of both kinds of errors are minimized. Additional systematic error sources are background scattered light, and secondary scattered light. Secondary scatter is Doppler shifted once from the flow seeding, but is shifted again an unknown number of times by additional particles with unknown velocities and directions. Reducing the light intensity or seed density is presently the only way to minimize this error, but this lowers the scattered light intensity, which decreases the signal to noise ratio.

Next, McKenzie examines in depth the effects of temperature and vapor pressure on the iodine cell frequency filter and its many transmission lines. The iodine cell has a main body where the light transmission takes place, and a small protrusion called a stem which is kept colder than the main body so that any crystal solidification will occur out of the optical path. The significance of the stem temperature on the depth and slope of a transmission line within tuning range of the pulsed laser is illustrated for purposes of broadening and steepening of the slope. In addition, if the transmission line is made deep enough to block all light of a certain frequency, the cell can effectively function as a notch filter. Studies done on the sensitivity of the line shape revealed that the shape is a very strong function of stem temperature, but only a weak function of main body 
temperature. Therefore, it is vital to control the stem temperature accurately since that controls the optical density which is indicative of the vapor pressure of the iodine.

A thorough error analysis follows, which concludes that the CCD camera array is the limiting error source. Even though the camera is a scientific grade, cooled, slow-scan, 12 bit camera, all other sources of error have been minimized to a point below the accuracy of the camera. This error analysis differs from that of Meyers, in that Meyers will test the system, then find places where the accuracy could be improved, usually through improvements to the data reduction software, after a thorough analysis of the data. McKenzie approaches the error analysis from a more theoretical point of view by creating uncertainty trends from variance data, which is obtained more often from theory. This is not meant to remove any practicality from McKenzie's study. Rather, his results stand as an ultimate limit for other researchers of what is possible with a similar planar velocimeter system.

The most recent effort from McKenzie (Jan, 1997, Sept, 1997) focused on a pulsed laser, planar system. Expanding on his earlier effort, a thorough error analysis was presented for the system as configured. Unique among his methods of reducing these errors is a 3 X 3 binning scheme whereby each pixel value in a 3 X 3 grid is replaced by the sum of all pixel values in the grid. This reduces many of the sampling and mapping errors associated with acquiring and overlaying two images read from a CCD array. A discussion of laser speckle is also presented, and an analysis of speckle noise contained in images collected at different focal lengths and apertures concludes that speckle noise is reduced in direct inverse proportion to the f-number of the receiving optics, as Smith had previously concluded. Average velocity measurements of a rotating wheel and a lowspeed turbulent jet were presented. Significant scatter was seen in portions of the wheel data, and it was attributed to a spatial variation in laser frequency across the beam, which was expanded to illuminate the surface of the wheel. It appears that this phenomenon is unique to pulsed-YAG lasers, and only affects the data in the case where the profile of the beam is enlarged. Measured RMS fluctuations of the jet flow were found by subtracting an estimate of the noise due to the laser and cameras from the total RMS of the signal. What remained was postulated to be due to turbulent fluctuations in the flow. Away from 
these variations, the minimum resolved velocity is approximately $2-3 \mathrm{~m} / \mathrm{s}$, which is the best accuracy published to date by anyone with a similar system.

Over a span of three years, Reinath (1997) made measurements of different flows in two different NASA Ames wind tunnels, including the 40 X $80 \mathrm{ft}$. full scale tunnel. The system used was a three component, six camera, cw laser system with a frequency monitoring leg. Because of the large distances involved in some of the tests, a powerful 8 watt argon ion laser was used in conjunction with a galvanometer scanner to create a uniform, high intensity light sheet. Optical access to the test section had a large influence on the geometry chosen for the tests, and the errors associated with angular resolution and transformation into orthogonal components showed the results of this limitation. In the early tests, the iodine cells were left uninsulated, causing the temperature, and therefore the partial pressure of the iodine vapor to vary. Since the cell calibrations were performed in the laboratory prior to the experiment, they were characterized at a different temperature than that of the test conditions, and as a result, there was an offset in the velocity data collected at the test site. In later tests, the cells were calibrated just before data was taken to yield a more reliable transformation.

Ainsworth and Thorpe at the University of Oxford (1994) published their plans to develop a three component DGV system to make measurements on stator/rotor flow interactions. Some initial test data in the form of images of a rotating wheel were also presented. Their experimental setup utilized a single camera with a 6-bit digitization frame grabber, split image system with a reference photodiode system to monitor laser frequency. Angular variation of the Mie scattering light intensity was analyzed and placement of the measurement components reflected this. Also, they have successfully documented the behavior of an argon ion laser operating in single frequency mode. They suggest caution be taken since the short term frequency fluctuations occur on a time scale which is on the order of a single CCD camera exposure. In this preliminary study, the only results presented are trends of ratio value across a vertical cut of a rotating wheel. No velocity comparisons were made.

The same Oxford group (Thorpe, et. al., 1995) published a more application orientated paper a year later, examining the flow of a free jet, as well as presenting 
velocity results of the same rotating wheel used in the previous paper. The imaging equipment used changed to a high grade, cooled scientific CCD camera linked to a 15 bit digitization frame grabber. From an analysis of error sources, error bounds were calculated for both the wheel and jet velocities. Since the velocity of the wheel was known, a comparison of the RMS deviation of the velocity and the error bounds could be performed. The results showed the error bounds to be several times larger than the actual error. They were used in the presentation of the pipe data, but identified as overly conservative. The presentation of pipe velocity data in this paper shows the DGV system to be qualitatively accurate, but stops short of a numerical data comparison.

A later paper (Thorpe, et. al., 1996) investigates the free jet further by timeaveraging the acquired images and carefully documenting the accuracy of their system. Time averaging was accomplished by opening the shutter of the camera for extended periods of time. In this manner, average velocity fields were attained by processing only one image. Measurements were taken with the light sheet perpendicular to the flow, as well as axially, down the center of the jet. In both cases, the sensitivity vector was in a direction which allowed the axial component to be found. Their detailed presentation of the velocity results shows a very good agreement with theoretical profiles of jet velocity. These researchers (Ainsworth, et. al., 1997) recently published a survey of the work done to date by themselves and others in the field of Doppler Global Velocimetry, highlighting the common trends and various differences of existing systems and methods.

An early paper by Chan, et. al. (1995) is one of the first to explicate the advantages of a single camera, split image DGV system. Simplified electronics, lower cost, and higher quality images are all potential benefits of adopting a velocity system configuration containing a single camera. Measurements of a rotating wheel were presented in terms of gray level ratio values and not velocity, presumably due to difficulty in establishing a reliable iodine transfer function. Instead, the focus of the work was more directed on reducing the variations and noise seen in the raw ratio images. Image alignment problems were identified as causing the majority of the low frequency variations from the expected linear trend of ratio values. To improve the pixel to pixel image alignment in the near field, the authors refined the procedure used to adjust the 
image position on the CCD array by viewing a speckle image formed by scattered laser light. When the speckle was viewed by a perfectly aligned system, the pattern was identical in both images. Painstaking alignment in this manner allowed the acquisition of data with a more linear trend. For suppression of the high frequency noise which was superimposed on the linear ratio measurements, two different digital filters were tested. The first filter was a 3 by 3 spatial averaging filter, which removed some of the high frequency content from the data, but introduced a bias in the trend of the variation. The second filter was a 3 by 3 median filter, where the center pixel in the array was replaced by the median of the pixel values of all nine pixels. The median filter was less effective at reducing the noise level, and also induced a bias in the data.

Clancy, et. al. (1998) researched a three-component system used to investigate a supersonic jet flow. Their system included a pulsed-YAG laser, and iodine vapor cells for frequency discrimination. The iodine vapor was pressure broadened with nitrogen to allow the absorption feature to span a wider range of frequencies, made necessary by the large Doppler shifts encountered in the flow. A single camera split image system was used in an attempt to minimize the cost while maintaining a high level of accuracy. Mean velocity as well as turbulence intensity results taken in both cross stream and streamwise directions were compared with LV data. A thorough error analysis was also presented which quantified errors associated with each aspect of the system. As with other researchers using pulsed lasers, one of the biggest contributors to the measurement error was found to be speckle noise. The approach taken in this work was to use a variation of a Weiner filter in post-processing. While this reduced some of the speckle noise, it also had adverse effects on the measured turbulence intensities and definition of features in the shear layer, as would most filtering techniques.

Work done by Reeder (1996) extended the split image concept to a twocomponent, one iodine filter, one camera system with four separate images captured on one CCD array. While this obviously reduced the spatial resolution for the data images, data taken for a supersonic jet showed velocities to within $10 \%$ of both PIV and pitot probe data, showing promise for the possibility of research of this measurement technique on a much smaller budget. 
Researchers from Texas A\&M University (Morrison, Gaharan, and DeOtte, 1994) developed a one-component DGV system based on the setup used by Meyers and developed by Komine. Their paper centered around the difficulties of setting up and obtaining accurate measurements from such a system. Problems included difficulty in setting the gains on the cameras purchased, inoperative or damaged CCD sensor elements, and non-uniform response to light intensity. Optics problems involving lenses, beamsplitters and polarization effects were also discussed. Additionally, they pointed out the importance of attenuating the scattered light that enters the reference camera using polarization filters rather than neutral density filters, on the assumption that the scattered light was completely polarized. However, other researchers have discussed the effects of particle scattering, and polarization, and they concluded that while the polarization of the scattered light may have a preferred direction, particle scattering interferes with the complete polarization of the incident laser light, producing a non-deterministic distribution of polarization. If care is not taken, the use of polarizing filters as neutral density filters may unintentionally discard a disproportional amount of scattered light leading to erroneous readings. Morrison, et al. also used a rotating wheel, but as a velocity calibration device. Other researchers used the rotating wheel to verify the accuracy of their measurements which were calculated based on the molecular characteristics of iodine and the geometry of the setup. By directly calibrating the velocity with light intensity, Morrison's group chose a much less complex method of resolving velocity data. However, using this type of calibration assumes that many variables not included in the calibration will remain constant. Consequently, the results of an experiment measuring the centerline velocity of a axisymmetric jet show wide scattering ( $\pm 20 \%)$ when compared with LV data.

The planar velocity imaging method most commonly yields three velocity components in different vectoral directions by focusing three systems of receiving optics on the measurement area and acquiring the data simultaneously. This is the only configuration that allows simultaneous acquisition of all three velocity components. However, if time-averaged data is all that is required, a different configuration is possible. Roehle (1996) used a three component system to measure flow exiting a swirl nozzle, as 
well as the flow field behind a scale model of an automobile. The components were found using a single imaging system and three different laser sheets, switched on one at a time. The imaging system used two 12 bit, slow scan CCD cameras focused on the same area through the use of a non-polarizing beamsplitter. Careful alignment, using micro positioning equipment, of the viewing angle of each camera precluded the use of any dewarping software to obtain corresponding pixel registration. The author plans to include such a dewarping scheme in future work. As in the point measurement system discussed earlier by the same author, active control of the laser frequency was achieved to within $1 \mathrm{MHz}$ of the setpoint. Control of the laser frequency of that precision nullifies the need for a frequency monitoring and compensation system. Roehle emphasizes the comparatively short time required to acquire and process a DGV measurement as the greatest advantage of the method. Less emphasis is placed on the accuracy of the data, indicating in the paper that an accuracy study is planned for the future.

Two pulsed lasers operating at different wavelengths are used in a two-color approach to DGV in Arnette, et. al. (1998). One laser emits green light at $532 \mathrm{~nm}$, and the other, red light at $618 \mathrm{~nm}$. Both beams are spread into a co-planar sheet and the scattered light is passed through an iodine cell and imaged on a color camera. The green laser light is tuned so that it is attenuated, by the iodine cell, in direct proportion with the frequency shift, as is the norm with DGV systems. The red light intensity in the images has no frequency dependence. Consequently, there is no need for two cameras or even one camera viewing a split image. The reference and signal images are both collected on one color CCD array. Velocity measurements taken in a compressible jet flow show potential for this type of system, although the increased cost of an additional laser may outweigh the benefit of needing one less camera or having a simpler data acquisition and reduction algorithm, making this novel approach cost prohibitive.

An alternative to the iodine cell as a frequency discriminator is presented by $\mathrm{S}$. Bloom, et al (1991). in their setup for a long range Doppler velocimeter. In this setup, a small telescope is used to collect the back scattered light from a laser diode. The telescope is field limited by placing a pinhole at the focal point of the primary mirror. The scattered light is focused through a polarizing beamsplitter, then each half is passed 
through a frequency discriminator. The filters are filled with atomic cesium, which, when heated and placed within a magnetic field, rotate the polarization by an amount proportional to the frequency of the light passing through it, the strength of the magnetic field, and the temperature. By placing polarizers rotated by $90^{\circ}$ on both ends of the cell, the intensity will vary with a change in frequency. The light is then focused on a photodiode for output to a data collection device. The other half of the light coming out of the beamsplitter is passed through identical optics, but in this case, the magnetic field is changed such that an absorption feature lies centered on the same frequency as the other cell, but with opposite slope. A system set up in this manner provides double the velocity resolution as does a filtered/unfiltered optics system. No cameras are involved with this system, but it is still a planar system; this system simply outputs the average velocity in the plane. The technical details of the cesium cell line filtering method are outlined in a prior paper by Menders, et al. (1991). An application of this technology is presented by Bloom, et al. (1993), and consists of long range detection of a helicopter plume.

Normally, when molecular iodine is used as the frequency discriminator, a cw argon-ion or pulsed Nd:YAG laser is used for the interrogating laser beam. Research done by Leporcq, et. al., focused on using a narrow bandwidth, tunable dye laser. This type of laser has a linewidth of approximately $500 \mathrm{kHz}$, compared to a $12 \mathrm{MHz}$ linewidth for a $\mathrm{cw}$ argon ion laser. An even more attractive feature is the ability of the dye laser to be tuned over a much larger frequency range, allowing the selection of an iodine absorption line to complement the experimental setup and expected velocity range. Rotating wheel data was presented to show the validity of the use of this type of laser.

Research similar to Bloom and Menders' is being conducted at Purdue University (Crafton, et. al., 1998), but with a point based system. The system under development there also contains a Cesium vapor Faraday cell functioning as a frequency discriminator instead of an iodine vapor cell favored by many other researchers. A large portion of their research has been focused on the establishment of a stable laser frequency for interrogation of the flows. To a large extent, this has been accomplished through the use of active PID controllers. Some improvement is to be made in the low frequency area of 
control of the laser diode. However, the high frequency control produced a stable enough laser frequency to record velocities with measurement errors under a meter per second.

Irani and Miller (1995) and Irani (1995) describe a single component system that used a cw argon ion laser with two cameras and an iodine cell. The authors used this system to measure, with some difficulty, a low speed jet seeded with model train smoke. Problems with keeping the laser frequency stable for any period of time prohibited acquiring an iodine absorption curve calibration, and the capabilities of the frame grabber system did not include simultaneous acquisition from the two cameras. Despite these serious limitations, measurements were made and compared to hot film and pitot probe data. Since no calibration of the cell was made, the comparisons were more qualitative in nature, but the data showed the potential of the system nonetheless.

All of the research discussed previously used particles to generate scattered light either by seeding the flow with various aerosols or relying on the flow's own contaminants. The amount of scattered light collected is proportional to the density of the seeding. Another planar velocimeter is being investigated by several researchers which involves a different method of scattering the laser light to the receiving optics. The laser light is scattered by the air molecules themselves in a process called Filtered Rayleigh Scattering (FRS). Miles, Forkey, and Lempert (1992) incorporate this method of scattering in their FRS velocimeter. In order to collect enough scattered light on the CCD arrays to analyze, the laser power must be very high. Currently, pulsed lasers are the only practical choice when very high power is needed. Continuous wave lasers can develop high enough power, but not when lasing in a single longitudinal mode, which is what is required for the type of velocimeter under discussion. When enough scattered signal is achieved, that signal can be interpreted as follows: the intensity of the scattered light is proportional to the density of the air, the linewidth is proportional to temperature (so called temperature broadening), and the frequency shift is proportional to velocity. Miles, et al. discussed in detail the structure of the iodine absorption lines and their sensitivity to stem temperature changes, and developed a theoretical model based on iodine spectroscopy data which predicts the major spectral features of iodine to within $5 \%$ of 
their peak values. Measurements were made in a Mach 5 free jet to test the system. Since the flow was at such high velocity, the absorption line was used to block all light entering the optics at the laser frequency and let pass only the Doppler shifted light. For this method to work, an optically thick iodine filter must be used. This is the same "notch filter" application as was described above.

In a follow up paper, Forkey, Finkelstein, Lempert and Miles (1996) analyzed the uncertainty of an FRS system, and made measurements of stationary room air and a Mach 2 jet. Temperature stabilization of the iodine cell, found to be critical in previous work, was also employed here. The transmission profiles of iodine as well as the laser lineshape and frequency were measured and accounted for. An uncertainty analysis quantified the error in laser wavelength, laser sheet distribution (line shape), stability of the laser frequency, background scattered light intensities, and the setup geometry. Total uncertainty was on the order of $5 \mathrm{~m} / \mathrm{s}$, and largely due to background scatter and laser drift. Errors of +31 to $-12 \mathrm{~m} / \mathrm{s}$ in the mean $0 \mathrm{~m} / \mathrm{s}$ room air were attributed to variations in lineshape as the frequency of the laser was changed, and errors of $15 \mathrm{~m} / \mathrm{s}$ were said to be due to the jet flow exiting the nozzle at an angle slightly different than $90^{\circ}$ to the nozzle. Future work involves employing an ultraviolet laser with cameras sensitive to that wavelength. The higher frequencies are beneficial to FRS because the amount of scattered light is proportional to the laser frequency to the fourth power.

More recently, Miles and Lempert (1996) examined various three-dimensional flow diagnostics and their relative merits. Techniques discussed included methods based on flow tagging and Rayleigh scattering. The tagging methods are based on passing a high intensity laser pulse through the fluid and either vibrationally exciting molecules in the fluid so that they briefly emit light, or activating a fluorescent additive which glows when interrogated by a second laser beam or sheet. The accuracy of both of these tagging methods is determined by the accuracy of the time delay and line displacement measurements and can be as good as $1 \%$ error for a single component velocity measurement. To extract the second and third velocity components as well as rotational information, the fluid may be tagged with two or more intersecting beams, then viewed from different directions by two or more cameras. Rayleigh scattering, in the manner 
previously discussed, is used to determine three orthogonal velocity components everywhere in the measurement plane. Volumetric velocity data can be obtained by rapidly scanning the laser sheet during an exposure.

Elliott, et al. (1994) are also researching an FRS instrument. Their system incorporates many of the same techniques and hardware as Miles' FRS system described above. The technique of using one pressure broadened iodine cell for velocity determination, and the other for suppression of background signals has been used in their setup. The cell is broadened by adding various amounts of nitrogen to the iodine vapor. The slope of the absorption line is decreased, and therefore the usable frequency shift is increased in an amount proportional to increase in the nitrogen partial pressure. The setup used to trace the shape of the iodine absorption curve used a small piece of the laser beam used to take measurements, and split it three ways. One of the three passed through the iodine cell to be mapped, and terminated at a screen. The second, serving as a reference, passed freely to a nearby point on the screen, while the third, which provided frequency information, passed through a confocal etalon and onto the screen. The three spots are then imaged with a CCD camera and analyzed. The fact that the same laser that is used for measurements is being used to interrogate the iodine cell leads to a calibration that is unique to that laser, and would be in error if a laser with a different linewidth were used for calibration. Measurements were taken in a supersonic/subsonic mixing layer where coherent structures are present, and were evident in the data taken. The errors in these measurements are quantified and discussed. They discuss the possibility of reducing the $\pm 8 \%$ error to about $\pm 3 \%$ when accounting for the laser drift by measuring it on a frame by frame basis as other researchers have done.

\subsection{Summary}

There have been many researchers involved in the development of DGV as a technology. Most, in recent years, are going through the work of constructing and testing their systems, without much attention paid to documenting the accuracy of the instrument. Recently, that has begun to change. McKenzie at NASA Ames and Clancy at Ohio State have both done thorough error analysis and velocity comparisons. It is the goal of the research outlined in this paper to add to this growing body of knowledge. 


\section{Chapter 3: APPARATUS AND CONFIGURATION}

This chapter will describe the DGV apparatus and setup configuration for both the rotating wheel and pipe flow tests. A brief description of this system, along with preliminary single component measurements on a rotating wheel, have been given by Naylor and Kuhlman (1998). The role and behavior of each of the system components will also be described. The overall principle of the DGV system is to measure the change in light frequency due to particle motion, and resolve that frequency shift into a particle velocity. The component sensitive to changes in frequency is the iodine cell filter, which possesses many narrow absorption lines, one of which lies within the tuning range of a cw argon laser. When the laser frequency lies on the edge of one of these lines, the filter has an attenuation which is a function of frequency. When the laser is set to a frequency that corresponds to partial light attenuation, variations of the intensity of laser light scattered from moving particles, as seen through the iodine cell, correspond to Doppler frequency shifts, laser light frequency variations, or simply changes in the intensity of the scattered light due to nonuniform seeding and uneven laser power output. The laser frequency and scattered light intensity are not constant, at least not to the degree needed to resolve velocity changes. To compensate for changes in laser intensity, part of the incoming scattered light is imaged on a photodetector or camera so that the signal intensity may be compared with a reference. To compensate for laser frequency drift, a portion of the interrogating laser beam is passed through another iodine cell such that the output of this reference system yields the frequency change in the unshifted laser light and can be subtracted from the frequency change calculated by the velocity measuring components.

The preliminary point-based system developed was an optical point measurement device which resolved velocities in two orthogonal directions. The basic elements of the system are similar to what was described in Hoffenberg (1993), and are outlined in more detail in James (1997). The planar, camera based system shares some of the same components with the point configuration, and a top view photo of a velocity measuring system can be seen in, Figure 


\section{$\underline{3.1 \text { Geometry }}$}

The placement of the laser beam and the velocity sensing optics relative to the flow is critical because the Doppler shift is a function of the scattered and incident light directions. This geometry dependence is contained within the Doppler shift equation given as

$$
\Delta f=\frac{f_{\mathrm{o}}}{\mathrm{c}}(\hat{\mathrm{a}}-\hat{\mathrm{l}}) \cdot \overrightarrow{\mathrm{V}}
$$

where $\Delta f$ is the Doppler change in frequency, $f_{\mathrm{o}}$ is the incident laser frequency, $\mathrm{c}$ is the speed of light, â is a unit vector describing the direction scattered light travels toward the receiving optics, $\hat{i}$ is a unit vector representing the laser propagation direction, and $\vec{V}$ is the particle velocity vector (Figure 3.2) The frequency change per unit velocity can be thought of as the sensitivity of the instrument, and is constant for a given geometry.

$$
\text { sens }=\frac{\Delta f}{\|\mathrm{~V}\|}=\frac{f_{\mathrm{o}}}{\mathrm{c}}(\hat{\mathrm{a}}-\hat{\mathrm{l}}) \cdot \hat{\mathrm{V}}=\mathrm{const}
$$

Maximizing the sensitivity provides finer velocity resolution, which has a positive effect on the RMS deviation due to noise. Since $\hat{a}$ and $\hat{l}$ are both unit vectors, their difference always lies halfway between the observation direction and the direction of the incoming laser beam. This vector is labeled as the measured component. It is important to visualize this angle so that the component of the flow under examination can be made to align or nearly align with the measured component. Adjustment of the measured component direction affects the dot product magnitude and is maximized when perfectly aligned with the flow direction.

The one remaining way to further increase the sensitivity is to maximize the length of the resultant vector of the difference between the laser direction and the viewing direction by making them as close together as possible. The ideal arrangement would be to have the laser and the receiving optics in the same position, so that scattered light would travel directly back along the laser beam (direct backscatter), with the flow moving either towards or directly away from the laser and optics. This setup is impractical for a number of reasons including physical size/space limitations, mie scattering intensity distributions, and the inability to view the scanned beam as a sheet; however, it serves as 
a reference of the maximum sensitivity achievable from a geometric standpoint.

The geometry used for wheel velocity measurements is shown in iFigure $3.3 ;$ The sensitivities for this configuration are 1.1 and $3.1 \mathrm{MHz} /(\mathrm{m} / \mathrm{s})$ for components 1 and 2, respectively. Component \#2 has a higher sensitivity because the viewing angle is more closely aligned with both the laser and velocity vector direction, producing higher values for both the subtraction and the dot product.

The original configuration for the pipe/jet flow velocity measurements was similar to that of the wheel. The expected velocity vector, however, was changed by more than $90^{\circ}$ with the substitution of the pipe apparatus. Also, the signal strength for the component in backscatter was less than half of the signal collected by the component in forward scatter. Consequently, the quality of the data for the component in backscatter suffered. In an attempt to equalize the signal received by both velocity measuring components, each component was placed in forward scatter at an angle of approximately $40-45^{\circ}$ on either side of the laser sheet (3igure $3.4 j$. The sensitivity value for components 1 and 2 were -1.2 and $1.3 \mathrm{MHz} /(\mathrm{m} / \mathrm{s})$ respectively. The sensitivity value for component \#1 is negative because it views the measurement area from the pipe side of the laser sheet. From this viewing angle the velocity vector is primarily opposite the measurement vector.

Viewing angles were referenced from the center of the measurement area to the center axis of the collection optics. Because DGV is planar in nature, the viewing angles will be different for points not at the center of the measurement plane. An analysis was performed to determine the extent to which these angle variations affected the data. The wheel geometry was chosen for the analysis because the wheel was the largest item tested, and also required the spreading of the laser beam into a cone, which changed the incident laser angle over the surface of the wheel. Therefore, the wheel test presented the largest potential for variable viewing angle error. Based on the geometry of 'Figure 3.3 ; there exists a maximum angle error (between opposite sides of the wheel) of approximately $7 \%$ for component \#1, and 3-4\% for component \#2. The sensitivities of viewing angles to the measurement of the velocity normal to the wheel are 1.1 and 0.38 $(\mathrm{m} / \mathrm{s}) / \mathrm{deg}$ for components \#1 and \#2, respectively. Tangential velocity sensitivities are 0.76 and $0.86(\mathrm{~m} / \mathrm{s}) / \mathrm{deg}$. Given these sensitivities and the maximum viewing angle error 
possible for each component, a maximum error of $8 \mathrm{~m} / \mathrm{s}$ (out of $57 \mathrm{~m} / \mathrm{s}$ ) occurs in the normal velocity measurement of component \#1. For this effort, the results ("cuts" through velocity images) are given as velocity values along the horizontal center, where no Doppler shift is measured, and the vertical center, where the viewing angle is referenced. Therefore, no significant viewing angle errors should be seen in the results as they are presented.

\subsection{Laser}

The laser used for this research is a Coherent Innova Model 305, continuous wave, 5 watt, argon-ion laser operating in single line mode at $514.5 \mathrm{~nm}$. The single line modification includes a prism mounted on one end of the laser cavity with a high reflectivity mirror aligned so that only one wavelength, or frequency, is reflected back along the resonance cavity. Each individual frequency may be selected by adjusting the tilt of the prism, but the strongest occurs on the "green line" at $514.5 \mathrm{~nm}$ which will output approximately 2 watts of power. Within the green line, there are many individual, closely spaced modes separated by a frequency determined by the laser cavity length and the speed of light, $\Delta \mathrm{f}=\mathrm{c} / 2 \mathrm{~L}$. The cavity length of the Innova 305 is $1.16 \mathrm{~m}$, which corresponds to a mode spacing of $129 \mathrm{MHz}$.

The numerous modes within the green line span almost $4 \mathrm{GHz}$, which is more than the entire width of an absorption line of molecular iodine, rendering frequency discrimination impossible. By inserting a tilted etalon into the laser cavity, single frequency operation is achieved. An etalon is a cylindrical piece of fused silica which, when slightly tilted, acts as a bandpass filter wide enough for only one mode, and the passed frequency is selected by changing the effective pathlength of the light undergoing many internal reflections inside the etalon. Filtering out all but one mode gives a very narrow linewidth $(<25 \mathrm{MHz})$, but since all other modes are not resonating, laser output power is decreased. The maximum power output of the Innova 305 with the etalon tuned to the highest power frequency in the green line is approximately $1.5 \mathrm{~W}$. Tilting the etalon produces very small changes in etalon length, and changing the etalon temperature changes the index of refraction, both of which affect the pathlength and therefore cause the etalon to pass different frequencies. 
When the preferred frequency changes, it does so quickly and is referred to as a mode hop. The tilting or heating of the etalon produces frequency changes, but they are discrete selections of the modes already present in the laser cavity. In a very basic sense, the etalon can be thought of as a selector switch on a TV, used to select a specific channel, but like a TV, only one channel can be tuned at a time. Since the spacing of the modes within the green line is equal to a constant $129 \mathrm{MHz}$, forcing the laser to mode hop produces a fixed, known, frequency change which can be used as a calibration standard for mapping the absorption line of the iodine cell. Conversely, an uncontrolled, continuous frequency change in the form of laser drift is unwanted. Laser frequency drift on the order of one mode spacing or less can be seen as the laser cavity length changes due to slight internal temperature changes. Presently, the only way to prevent this drift is through active cavity length adjustment, which monitors the laser frequency and adjusts the cavity length to maintain a specified frequency. A less complex solution, which has been employed in this research, is to measure the drift and compensate for it.

\section{$\underline{3.3 \text { Iodine Cells }}$}

Using the evenly spaced mode hops to map out the iodine absorption line works well only if the vapor pressure of the iodine in the cell remains constant during the calibration and subsequent data collection. The cells used in the present work have been constructed by Opthos Instruments, Inc. to the specifications of cells made previously for the researchers at NASA Langley (Meyers, 1996). The cells are 2.5 inches long and 2 inches in diameter with optically flat anti-reflective coated crown glass fused to the ends. They are first evacuated, then a few iodine crystals are placed inside and the glass stem (nominally 0.5 inches long) is melted shut. The iodine crystals undergo sublimation until the volume is saturated with vapor. Since the iodine cells are sealed, the only way to increase or decrease the vapor pressure is to change the temperature. Therefore, in order to keep the vapor pressure, and hence, the absorption profile constant, the temperature must be kept constant. To this end, each iodine cell is fitted with a 3/8" thick cylindrical copper sleeve, slightly longer than the cell, around which two electric band heaters are placed. The copper serves to evenly distribute the heat transfer to the cell from the bands, and it also provides a large thermal mass, aiding the operation of the temperature 
controller. A picture of an iodine cell with copper jacket and thermal ground visible is shown in 'Figure 3.5i. The controller is an Omega CN9000A Miniature Autotune Temperature Controller. It is a PID controller, with user adjustable gains, but it can also be set to automatically select the proper gains when operated in autotune mode. When the controller was configured in the autotune mode, the resulting temperature control had less than the desired stability. Consequently, the controller gains were manually adjusted until the proper response was achieved (Figure 3.6). The response has a long settling time ( 40 min.), but long-term stability, shown in more detail in Figure 3.7; was needed more than a fast settling time. The use of an accurate temperature controller allows the flexibility to set a desired temperature and have it maintained throughout the calibration and data collection.

When the temperature of the cell is elevated, the iodine will tend to solidify in the cooler regions. For optical reasons, it is not desirable to have crystals form on the windows, which would ordinarily be the coolest points on the body of the cell. To prevent these deposits, two 1.5" lengths of thermally insulating phenolic tubing are inserted in the ends of the copper jacket and capped with anti-reflective coated, optically flat, crown glass. This provides a buffer of insulating air, keeping the cell windows at a higher temperature than the stem temperature. With this arrangement, solid deposits are not allowed to form anywhere on the cell body, so a place must be available for crystals to form. This space is furnished by the stem originally used to fill the cell. One end of a heavy gauge stranded copper wire is bonded to the outside of the stem to function as a heat sink, while the other end is fastened to the optical breadboard. The wire cools the stem temperature to approximately $10^{\circ} \mathrm{C}$ lower than the body temperature. It was found by McKenzie and others that the vapor pressure was a very strong function of the stem or crystal temperature, and a very weak function of the body or vapor temperature. Therefore, the thermocouple that the temperature controller uses for feedback comparison to the setpoint is bonded to the stem, and the body temperature is allowed to reach whatever value required to provide enough heat to the stem to keep its temperature equal to the setpoint. To further insure a stable cell temperature, the entire device is surrounded by an insulated box with holes cut for the phenolic tubes. The long-term temperature 
variation (Figure 3.7) of this system during summer months with the building air conditioning running, has been shown to have a standard deviation of less than $0.1^{\circ} \mathrm{C}$ (peak-to-peak of $0.5^{\circ} \mathrm{C}$ ) with a room temperature fluctuation of $1.5^{\circ} \mathrm{C}$. Room temperature variations during winter months are typically $4^{\circ} \mathrm{C}$ over a 40 minute period, while measured stem temperature variations are similar to those in Figure 3.7 It is important to note that the time scales for calibration and data acquisition are more on the order of the short term fluctuations, and therefore, the standard deviation is more representative of the performance of the controller for this application. The iodine cell control and insulation was developed for the reference system cell, and has been duplicated for the signal systems.

Since the body temperature is held approximately $5-10^{\circ} \mathrm{C}$ higher than the position of the stem where the thermocouple is bonded, there exists a fairly steep temperature gradient over the length of the stem. Therefore, the placement of the controller feedback thermocouple relative to the solid iodine crystals is critical. When the thermocouples were bonded to the stems, no effort was made to maintain consistent placement along the length of the stem. Also, stem lengths vary somewhat from cell to cell. As a result, even though the temperature controllers were all set at the same setpoint, the iodine crystal temperatures were all different. This can be seen in Figure 3.8 i where the calibration curves from the three cells have been normalized and overlaid. If all three cells contained an identical amount of iodine vapor, the curves would be the same (assuming negligible optics effects). The most pronounced difference in these curves is the variation of slope in the vicinity of the linear region, which is a primary indicator of a difference in crystal temperature.

\subsection{Reference System}

The function of the reference system is to monitor the frequency of the laser beam. A layout of the components can be seen in Figure 3.9 A small portion (approximately $8 \%$ ) of the main beam is split at a $90^{\circ}$ angle by a placing a 2 " round piece of Pyrex ${ }^{\circledR}$ glass in the path of the laser beam. The glass is untreated; that is, it is not coated with any antireflective material, so multiple beams are split from the front and back surface of the glass. Next, the multiple beams pass through another 2" piece of Pyrex ${ }^{\circledR}$ which splits off 
a portion and sends it to the spectrum analyzer. The second piece of Pyrex ${ }^{\circledR}$ is identical to the first, so that the number of strong (first order reflection) beams reaching the spectrum analyzer is doubled. Continuing past the second Pyrex ${ }^{\circledR}$ beamsplitter, the beam passes through a $1 \%$ transmission neutral density filter, then hits a beam selector plate which is nothing more than a piece of aluminum with a hole drilled in the size of one of the beams $(\sim 2-3 \mathrm{~mm})$. The beam that is not blocked by the plate is sent through a 50/50 beamsplitter for passage through both the reference and signal legs of the system.

The entire system is placed under a $1.5^{\prime} \mathrm{X} 5$ ' wooden box. The box is dual purpose; as a light box, it blocks all outside light except for a portion of the laser beam that is sent through a small (1/2") hole in the side of the box. As an insulator, it serves to lessen the variation in temperature of all the components inside the box, including the photodetectors which have a response to light that is slightly temperature sensitive.

On the reference leg of the system, the beam is turned $90^{\circ}$ by a $1 "$ front surface mirror, then focused by a $2 "$ diameter, $100 \mathrm{~mm}$ focal length (f 2 ) lens on a photodetector. The photodetector was purchased from Thor Labs as an amplified, but fixed gain, silicon diode device. It has a maximum output of $1.5 \mathrm{~V}$ and a bandwidth of $50 \mathrm{MHz}$. A $1 \%$ transmission neutral density filter is placed ahead of the 50/50 beamsplitter in the beam path to prevent photodetector saturation. With the neutral density filter in place, and the laser tuned for maximum power, the photodetectors have a nominal output of $0.8 \mathrm{~V}$.

For the signal leg, concerns about saturating the iodine cell were addressed. That is, by passing the laser light as a concentrated beam directly through the iodine vapor, the absorption characteristics would be a function of laser power. To ensure functional independence from laser power, the beam is first passed through a CVI Instruments 10:1 beam expander. CVI was chosen because the desired exit quality of the beam did not require the purchase of an expander with tighter tolerances and higher price. The nominally $2 \mathrm{~mm}$ diameter laser beam enters through a small hole in one end, then after passing through a series of lenses, exits as a parallel beam $2 \mathrm{~cm}$ in diameter. It then continues through the iodine cell and is focused by a lens-photodetector combination which is similar to that used in the reference leg.

The two voltages from the signal and reference photodetectors are simultaneously 
sampled with an IOTech 16-bit IEEE 488 interfaced A/D board set to a range of $\pm 1 \mathrm{~V}$. The maximum sampling rate supported by this board for two channels is $50 \mathrm{kHz}$. First, a reading is taken from each photodetector when all light is blocked from striking the sensing elements. These "dark voltages" are then subtracted from all subsequent readings acquired from their respective channels. The signal voltage, now due only to scattered light, is divided by the reference voltage to yield a percent transmission for the iodine cell. Once the laser is tuned to the slope of one of the iodine absorption lines, any change in the ratio corresponds to a change in laser frequency. Typical short term laser frequency drift can be seen in Figure 3.10 which shows the ratio of the voltage output of the reference system photodetectors. Similar short term frequency fluctuations were documented by Ainsworth and Thorpe (1994), also for an argon ion laser. The data have been acquired over the time span that a single field is acquired, $1 / 60^{\text {th }}$ of a second. The board is set to the maximum sampling rate $(50 \mathrm{kHz}$ for 2 channels) and the number of samples adjusted so that the acquisition lasts the required amount of time.

The photodetector selection for the reference system was intended to mirror the components that had been successfully installed in the DGV system in operation at NASA LaRC. The Thor Labs' PDA-150, fixed gain photodetectors were purchased in error, and were thought to be the variable gain type, PDA-50. When used for the reference system though, where the beam is steered directly into the photodiode, their amplification provided sufficient voltage for A/D conversion. Testing of the photodetector performance revealed the PDA-150 to have a smaller level of noise than the PDA-50s, but the PDA-50s were required in the velocity sensing systems of the point measurement system, given the low light intensities of scattered light. The PDA-150s remain installed in the DGV reference system, since light intensity is not an issue, and their signal to noise ratio is the same or better than the PDA-50s.

Prior to striking the beamsplitter for the reference and signal legs of the frequency monitoring system, the two strong beams ( $2 \%$ of main laser beam), and many much weaker beams, are split again with a 2 " diameter piece of Pyrex ${ }^{\circledR}$. The primary reflections from the Pyrex ${ }^{\circledR}$ are sent to the spectrum analyzer, but only a portion of one beam enters the analyzer through a pinhole aperture mounted on the front. The spectrum 
analyzer system (SAplus) was manufactured by Burleigh Instruments and uses a confocal Fabry-Perot interferometer consisting of two spherical mirrors spaced apart a distance equal to their radius creating a bull's-eye interference pattern. This arrangement was touted to have a higher precision than similar analyzers made by Spectra Physics. A measurement of precision or resolution $(\mathrm{R})$ is given by the finesse $(\mathrm{F})$ and the free spectral range (FSR) by $\mathrm{R}=\mathrm{FSR} / \mathrm{F}$. The finesse is a parameter than quantifies the quality of the instrument and its ability to measure the laser lineshape. The confocal mirror set is constructed to have a $2 \mathrm{GHz}$ FSR, and the mirrors are of sufficient reflectivity (>99.5\%) to provide a maximum finesse of approximately 300 , giving the analyzer a $6-7 \mathrm{MHz}$ resolution. The highest finesse indicated for the argon ion laser being used was approximately 100, which gives some indication of the laser's linewidth ( 20MHz). To output a useful electrical signal, the bulls-eye interference pattern is scanned across the surface of a photodiode by peizo-electrically vibrating one of the mirrors, causing the interference pattern to move. The output of the photodiode can then be amplified and displayed on an oscilloscope or sampled and analyzed by a computer. The most useful configuration involved the use of the oscilloscope to monitor discrete frequency changes during continuous scan calibrations to optimize the rate of etalon tilt.

\section{$\underline{3.5 \text { Velocity Sensing System }}$}

The velocity sensing system (Figure 3.1) is functionally the same as the reference system in that it monitors the frequency of laser light. The difference lies in the fact that it monitors the frequency of Doppler shifted, scattered, laser light. Light scattered from a seeded flow within the plane of the laser sheet passes through and is reflected by a 50/50 beamsplitter. The portion that is passed through the beamsplitter continues through the iodine cell and into a zoom lens attached to the front of a (signal) CCD camera. The portion reflected from the beamsplitter is turned on a front surface mirror which steers the image directly into another (reference) lens/CCD camera combination. The CCD cameras used are Hitachi KP-M1 black and white, interlaced cameras with 8 bits of resolution. The frame rate is fixed at $30 \mathrm{~Hz}$, or $60 \mathrm{~Hz}$ for fields. The zoom lenses used are Nikon $35-135 \mathrm{~mm}$ focal length lenses with a minimum f-number of 3.5 at $35 \mathrm{~mm}$, and 4.5 at $135 \mathrm{~mm}$. Zoom lenses allow flexibility in positioning of the receiving optics as well as 
selection of the spatial resolution desired, typically at the cost of reduced collected light when compared to similar focal length fixed lenses. The zoom lenses attached to the signal cameras have always been operated with the aperture fully open, and the apertures for the reference cameras have been adjusted to approximately match the intensity of the signal cameras when the laser was tuned outside the iodine absorption band. Four-inch optics were used for the beamsplitter and turning mirror so that a sufficient clear aperture could be obtained when the optics were angled. The optics have been held with 4" gimballed mounts with fine-adjust micrometer type screw adjustments. The beamsplitter is a dielectric plate-type, 4 " in diameter and approximately 0.1 " thick. It has been found that the gimballed mount that held the beamsplitter put too much force on the three points of contact around the perimeter, and bending of the plate glass produced a warped image. The springs were subsequently removed and shortened to gently hold the glass in place, but there still existed, due to slight bending, a significant amount of image distortion due to the beamsplitter which, in addition to the perspective warping, had to be removed by image processing. The 4" mirror did not have this problem since it was deposited on a 1" thick glass substrate. Also, the dielectric beamsplitter was polarization sensitive, splitting vertically and horizontally polarized light by different amounts. Since polarization of light scattered off particles is known to be size dependent (Meyers, 1997), the ratio of light split by the beamsplitter would change as a function of particle size. No effort has been made to ensure uniform particle size, so for seeded flow measurements, polarization filters were placed in front of the beamsplitters to block all but the principal polarization direction so that the beamsplitters would split the incoming scattered light at a constant ratio.

\subsection{Computer Related Equipment}

\section{Computers}

The computers used in this research were IBM compatible, personal computers (PCs) with Windows 95 and Windows NT as operating systems. The manufacturer of the frame grabber board required that Windows NT be the operating system for the computer housing their board. That computer had a Pentium II processor with a clock speed of 266 $\mathrm{MHz}$, and $96 \mathrm{MB}$ of memory installed. Also, a 4 GB hard drive was added for storage of 
image data acquired and subsequently processed with the frame grabber. The other computer, also a PC, ran Windows 95 on a Pentium processor at $200 \mathrm{MHz}$. This computer was responsible for acquisition of both temperature and photodetector voltages, using two different A/D boards. Since the size of the data collected with this computer was minuscule $\left(\sim 10^{2}\right.$ bytes) when compared to the image data collected by the first computer $\left(\sim 10^{9}\right.$ bytes), no special consideration was given to memory or hard drive requirements. All data, calibration, and correction images, acquired with both computers, were archived on recordable CD-ROMs. This allowed examination of past data for referencing and re-processing, if necessary.

\section{$\underline{\text { A/D Boards }}$}

Two A/D boards have been used to collect various data for the DGV system. The IOTech board, introduced above, is a 16-bit, simultaneously sampling, 8 channel, variable gain, external board with $100 \mathrm{kHz}$ maximum sampling rate divided between the number of selected channels. Also available externally, with a separate connector, are 8 digital input and 8 digital output lines, one of each used for status indication and synchronization control with the frame grabber board. The digitized data is brought to a PC via an IEEE 488 data bus and controller card. The controller card, which brings data from the IEEE bus to computer memory, is a National Instruments AT-GPIB/TNT and is capable of transfer rates of up to $1 \mathrm{MB} / \mathrm{sec}$. The maximum output of the IOTech board, which is the only device connected to the controller card, is $200 \mathrm{kB} / \mathrm{sec}$.

The second $\mathrm{A} / \mathrm{D}$ board is an internal model made by National Instruments, and is also 16-bit and variable gain, but has a slower sampling rate of $20 \mathrm{kHz}$ maximum over 8 channels, and it does not sample simultaneously. Simultaneous sampling provided by the IOTech board is necessary when sampling photodetectors so that the signal from each detector pair will be correlated and like variations will cancel when divided. The slower, non-simultaneously sampling board has been used to collect temperature data from thermocouples mounted on the body and stem of the iodine cells. Since these signals have much longer time constants than the $50 \mathrm{MHz}$ bandwidth photodetectors, high speed simultaneous sampling was not necessary.

IOTech's ADC488/SA was purchased to read the signals from all photodetectors 
because the specifications showed that, at the time, it was the least noisy 16 bit A/D board with the highest sampling rate. However, during initial testing, when the inputs were directly grounded at the terminal, a large noise signal was found, apparently internal to the device. 'Figure 3.11 is shows a comparison of the outputs of the two 16-bit A/D boards in use for this research. For both boards, the inputs only to channel one were grounded and 1000 samples were taken at each board's maximum sampling rate. The other channels were left ungrounded since the data was also acquired with this configuration. This test shows the relatively large noise levels inherent to the measurements made with the IOTech A/D board. However, this noise is not likely to have affected the DGV data, because the cameras used to acquire the velocity data digitized the intensities with 8-bit resolution, and even with a noise intensity of 6 bits, the board still had 10 bits of effective resolution. In the point based system, where there was no other device limiting resolution, the reduced resolution of the IOTech board had a significant impact.

Frame Grabber

A frame grabber is essentially a high speed A/D converter with specialized triggering and interface hardware attached. For example, for a typical CCD image with 640 picture elements (pixels) horizontally and 480 pixels vertically, with frames grabbed at video rates of $30 \mathrm{~Hz}$ and sampled at 8-bit digitization, the frame grabber must throughput data at a rate of $9.216 \mathrm{MB} / \mathrm{sec}$. For DGV applications, where multiple cameras need to be sampled simultaneously, the data rate is multiplied accordingly. These large data rates have only become possible in the last few years, and this performance requirement drove the selection of a high-end board manufactured by Matrox, Inc. called the Genesis. The Genesis was, at the time of purchase, the only board available on the market that was able to sample 4 CCD cameras simultaneously, and digitize them individually. It also features a parallel processor integrated on the board that allows image operations to be performed much faster than if carried out using the host computer alone. Local to the board is $16 \mathrm{MB}$ of memory, approximately $14 \mathrm{MB}$ of which is useable after initialization and device allocation. This memory can be allocated for $30 \mathrm{~Hz}$ acquisition and storage of 47 images, or 11 sets of 4 simultaneous grabs at the maximum camera rate of $30 \mathrm{~Hz}$. In addition to having 4 analog inputs, the board also 
accepts input from digital cameras and other non-standard cameras such as line-scan CCD devices.

\section{Traverse}

A three degrees of freedom computerized, motor-driven traverse was constructed to facilitate mounting and positioning of experimental apparatus. The traverse allows approximately $1^{\prime}$ of movement in the $\mathrm{z}$ direction (up-down), and within a 2 ' by $1 \frac{1}{2}$ ' rectangle in the xy plane. The traverse was designed and built by S. Ramanath (see Figure 3.12, using steel beams for the base and aluminum beams for the remaining structure. Using stepper motors for movement, translations on the order of inches are repeatable to within 0.001 inches in the same direction, but increase to 0.01 inches when a change of direction is involved, due to hysteresis. The ability to move the experiment rather than the optics and laser is pivotal in keeping the measuring volume aligned with the receiving optics. 


\section{Chapter 4: DATA ACQUISITION AND IMAGE PROCESSING}

The software written for all data acquisition, image processing, and the operation of the frame grabber is a mixture of $\mathrm{C}$ and Visual Basic. Visual Basic provides the frontend for all grabbing and processing DLLs, which have been written in C. The acquisition hardware for the reference (photodiode) system is housed in one personal computer, while the frame grabber is installed in a different, but nearby, PC. Additional details of the software may be found in Appendix A.

\section{$\underline{4.1 \text { Synchronization }}$}

The problem of synchronizing the photodiode and camera based acquisitions for the acquisition of velocity data has been solved through the use of digital inputs and outputs on both systems. The Genesis frame grabber board has two logic-level ports which can set or read a binary value. These two ports are connected to the first two bits of an 8-bit digital I/O port on an IOTech 16-bit A/D board. The connections form IO1 and IO2, which can be thought of as simple digital communication lines indicating a ready state for each acquisition device. Handshaking ensures that both systems are ready before any data are acquired. An example of this handshaking is outlined in 'Figure 4i, and is described as follows: the software for the A/D converter is started and the board is set to continuously monitor IO1, triggering the predefined data acquisition task when it goes high. When the board is done initializing, it sets IO2 to high to indicate a ready state. After the frame grabber board software is started and the initialization is complete, it checks the status of IO2. When IO2 becomes high, if it is not already, the frame grabber board sets IO1 high and starts a frame acquisition, triggering a simultaneous acquisition by the A/D board. Each system immediately resets the corresponding ready bit to low and repeats the process.

When the signal is given to start acquisition, the frame grabber simultaneously grabs one field (half of a full image--due to interlacing) for each camera, while the A/D

board samples the reference photodiodes for the same $1 / 60^{\text {th }}$ of a second. The A/D board will acquire 833 points during this time, but only record the average ratio value such that there is one reference ratio for every image field acquired. Because one field is taken after another, $1 / 60^{\text {th }}$ of a second apart, the flow is likely to move a significant amount 
between the first and second field acquisitions. For this reason, the two fields that make up an interlaced image cannot be used as one velocity image in DGV. Instead, the first field of each frame is kept and recorded so that in post-processing, the missing lines in the acquired field are filled in with the average of the pixel values directly above and below the empty line, creating a full frame to be analyzed. The other option would be to remove half of the lines in all of the correction images, then process the data fields as required. This option was not implemented.

The cameras, by themselves, are free running. That is, they provide their own horizontal and vertical sync pulses. In order to simultaneously acquire data from all four cameras, the internal sync pulses were overridden with externally supplied horizontal and vertical sync pulses generated by the Genesis board.

\section{$\underline{4.2 \text { Iodine Cell Calibration }}$}

Calibration of the iodine cell absorption line is important because an accurate plot of intensity ratio vs. frequency is needed to ensure reliable frequency measurement and accurate velocity results. Calibrations were performed on each cell to account for slight variations in iodine cell stem temperature as well as any optical differences that may exist. Iodine cell calibrations were accomplished using a continuous scan of the mode structure of the Argon ion laser operating on the 514.5nm (green) laser line, which is comprised of many resonant modes which are narrower in frequency by approximately 20-30 times than the width of the green line. By mechanically altering the tilt of the etalon through about 10-20 modes, the transfer function for one side of the absorption feature can be resolved.

A coarse calibration can be done by mode hopping the laser and recording the cell transmission ratio at each selected frequency. The reference system needs no modifications to do a mode hop calibration, and the velocity system needs only to be focused on a non-moving object which scatters the laser light. With this setup, the laser etalon is tilted such that the laser frequency lies at a point in the iodine absorption spectra of maximum transmission, and a set of ratios is taken and averaged. Then the laser is forced to resonate at a different mode frequency by slightly tilting the etalon via a screw adjust at the rear of the laser and data is taken again. The selection of consecutive modes 
is continued until the transmission through the iodine cell is at a minimum. The total range of the etalon is 30-40 mode hops, but one side of the iodine transition line is only 810 modes, or $1.032-1.29 \mathrm{GHz}$ wide (iFigure 4.2 ). A curve fit is performed to fill in the gaps.

A modified calibration procedure has been used in favor of the discrete calibration described above. The preferred procedure makes use of a longer data collection time of 30 seconds, during which, the etalon is tilted "continuously" to scan the laser frequency through the frequency range of one side of the iodine absorption well. Both images and photodiode voltages have been acquired over the 30 second period, where 60-80 image sets and 1500 photodiode voltages were recorded. Optimally, the scattering medium is the same as that which is used for subsequent testing, helping to maintain consistency between the calibration and the velocity data. Occurrence of mode changes was detected by a sudden jump in reference photodiode voltage and reference camera gray level. When the ratios are plotted with time, the data has a stair step trend with several data points forming each 'step' ('Figure 4.3i). Ideally, the data would be constant from mode to mode; ie, the steps would be flat. However, the process of tilting the etalon produces changes in the laser frequency, most likely due to small changes in the length or shape of the laser cavity, that manifest themselves as fluctuations in the ratio data within a single mode. Heating and cooling the etalon to produce the required length changes would most likely have less of an adverse effect on the stability of the laser cavity. However, the time required to change the temperature of the etalon enough to span a side of the absorption well, is long enough to allow external factors (namely ambient temperature and cooling water) to affect the overall frequency drift. In order to minimize the effect of the frequency changes within a mode, the ratio value for any one mode was computed as an average of all ratio data that were collected while the laser was in that mode. Earlier experiments were conducted on cell calibrations, within the context of the point system, where the values at the left and right ends of a mode were compared with the average value, and the best, most consistent results were obtained using the average value (James, 1997). It was also found that this continuous scan mode hop calibration technique offered better accuracy than an earlier technique involving discrete mode changes 
followed by many averaged data points for each mode. It was more accurate because it took less time to perform a scan, allowing less chance for the laser frequency to drift. Also, the effects due to variability of where one stops the mechanical screw adjust on the etalon tilt screw are minimized by this technique.

Significant further improvement in calibration accuracy, through a more representative curve fit, has been obtained by using several individual continuous scan mode hop calibrations together (James, 1997). This improved calibration consists of several (from 7 to 10) continuous mode hop calibration data sets for each cell. A single cell calibration data file is formed by "sliding" all mode hop calibrations for any one cell, to overlay them on one arbitrarily-selected calibration scan of the set. This procedure is accomplished by linear interpolation, and is necessary because of arbitrary starting points for each calibration data set, as well as temperature induced laser frequency drift between mode hop calibrations. After the calibration data are shifted, a best-fit curve is found in order to determine a relative frequency given a measured ratio. The most consistent method of fit was found to be a form of a Boltzmann fitting function. Other curves tested include, an $\mathrm{n}^{\text {th }}$ order polynomial and a stretched and shifted curve generated by theoretical means (McKenzie, 1995).

The form of the Boltzmann fitting function used is as follows:

$$
y=\frac{A_{1}-A_{2}}{1+e^{\left(\frac{x-x_{0}}{D_{x}}+A_{2}\right)}}
$$

where $A_{1}$ and $A_{2}$ are the top and bottom boundary ratio levels, respectively, $x_{0}$ is a horizontal shift, and $\mathrm{D}_{\mathrm{x}}$ is a horizontal stretching coefficient. This function is used heavily in neural network algorithms as an activation function. The function is fit to the data by varying the four coefficients until the error between the data and the curve is minimized. A non-linear, least squares, iterative method called Levenberg-Marquardt was used to perform the minimization (Press, et. al., 1992). An example of this curve fit, shown with the calibration data for each of the three iodine cells can be seen in'Figure't individual data points show less scatter than McKenzie has shown for calibration using a pulsed YAG laser (1997), especially for the photodiode data. The camera data show more scatter, but that is because significantly fewer points were used $\left(\sim 1 / 10^{2}\right)$ to 
calculate an average value for each mode. The curve fits the data very well in the middle, and less so near the top and bottom of the curves, which is why care is taken to acquire velocity data near the middle of these curves. Figures 4.5 and 4.6 compare two calibration curve fits for each of the two velocity measuring components. The curve fits were applied to data taken on two different days. The expanded portion of the curve more clearly shows the difference in slopes of the two fits. iFigure 4.5 contains the curve fits for component \#1, with a maximum velocity change of $1.9 \mathrm{~m} / \mathrm{s}$ due to frequency differences at maximum data ratios (up to \pm 0.15 ). The fits for component \#2, in:Figure $4 . \overline{5}$,, , show less frequency difference at the extreme ratio values. The maximum velocity error for component \#2 is $1.4 \mathrm{~m} / \mathrm{s}$. The geometry used to calculate these velocity errors was the same as shown in'Figure 3.3 .

The calibration procedure for the DGV system has an added step for the cells that are calibrated with video cameras as opposed to photodiodes. As in the point system, voltage data from the reference system photodiodes is acquired continuously while the laser is mode hopped through an iodine absorption line, but images through the other two cells are acquired as quickly as possible ( 2 images/sec). Then, the average gray level within a user-defined area for each calibration image is found and recorded before further ratioing, data shifting and curve fitting can take place. In this way, the cameras operate as very large, slow, lower resolution photodiodes.

A typical calibration scan takes 30 seconds to complete, while a set of 10 scans can be acquired in 10 to 20 minutes, depending on the behavior of the laser during the scans (scans for which a mode is skipped are discarded and repeated). Therefore, cell stem temperature drift is not as much a factor over the course of a single scan as it is during the acquisition of an entire calibration set. Over the course of a single scan, the frame grabber grabs images at a rate that allows 3 or 4 images per mode, whereas the A/D board acquires approximately 100 data points during a single mode for the reference cell. Consequently, after averaging over a mode, data from the cameras is less consistent than that from the photodiodes. Use of a larger number of individual scans in the curve fit process helps to correct for this inconsistency.

Some researchers skip an experimental calibration altogether, and instead use a 
theoretical model that predicts the absolute frequency of the absorption line based on cell body and stem temperature (Meyers, 1996). Others use a combination by fitting the experimental calibration with the theoretical model (McKenzie, 1995). If the theoretical model is used alone, absolute velocities can be calculated directly from photodetector ratios, provided accurate temperatures are available. In a mode hop calibration, no absolute frequency is known; therefore, only a comparison between the reference and signal cell calibrations can give an absolute velocity. However, temperatures must remain constant between calibration and data collection. Since this is very difficult to achieve, an apparent velocity offset is produced.

\subsection{Image Processing Procedure}

Two main goals of the image processing software are to more accurately represent the imaged area and to better align the views of the signal and reference cameras. A block diagram of the data reduction process is shown in 'Figure 4.7\% Most of the steps shown closely follow the comprehensive image processing methods developed at NASA Langley by Meyers $(1992,1996)$. Besides the cell calibrations, several additional images of a corrective nature need to be taken before each data run, while the system remains undisturbed. In each case where the target is stationary, several exposures are taken and averaged for each camera.

\subsubsection{Intensity Corrections}

\section{Background}

The first of these additional images is the background image. The background image is an image (average of several frames) of the data area with laser illumination but without seeding. Contributing to the apparent background light level is the camera dark current, which is due to the spontaneous generation of electrons in the CCD elements without photon stimulation, and appears as additive charge on top of the output image. The background light level can be partially reduced by cooling the array, or, as was done in this work, the sum of the dark current with the image of the test area can be subtracted from each data image to more completely compensate for these effects. The background images must be removed from the raw data image before the reference and signal pixel levels are divided. If not subtracted, the background scattered laser light represents a 
constant added to the particle scattered light signal, which does not cancel when dividing. Background scattering is more of a problem when using cameras as the light intensity sensing devices, because the depth of the field of view cannot be limited through the use of a pinhole, as it can be in a point based photodetector system. An improvement in signal from the point system photodetectors has been seen when the velocity sensing systems were covered with a black drape and boards blocked light from the front and back. Light has only been permitted to enter the system via a hole in the front masking board. Similar arrangements have been made for the reference system by covering it with a specially constructed box. This configuration has been preserved for the planar system.

An alternative method of minimizing the background light error, presented by Elliot, et. al. (1994), is to place an additional $I_{2}$ filter in front of all receiving optics. The filter is constructed such that light that is not Doppler shifted is completely attenuated, and Doppler shifted scattered light from the particles passes through to the receiving optics, effectively removing background scattered laser light from the image. The flow being measured must produce a large enough Doppler shift such that a fraction of the shifted light is passed through the cell, while the incident laser light is not. This method is most applicable in systems measuring high speed flows where the interrogating laser is of high power pulsed type and there exist many reflections from objects in the test environment, such as a wind tunnel model.

White Card

An averaged image of a laser-illuminated white card is also recorded. Laser light is used, rather than white light, in an attempt to closely emulate test conditions. Also, the laser is tuned such that the frequency does not fall in an iodine absorption line during this acquisition (McKenzie, 1997). The signal and reference images of the white card go through the same processing steps as do the data images up to and including the ratio step. After the division of signal and reference images, each channel's white card ratio array is normalized with respect to the average ratio value. The resulting matrix of floating point numbers should, ideally, be equal to 1.0, but imperfections in the imaging system (lenses, beamsplitters, mirrors, and cell ends) will cause variations in the light intensity, and therefore, the ratio. In particular, interference fringes produced by coherent 
laser light reflecting off nearly parallel glass surfaces of the iodine cell affect the light intensity of the signal camera, and must be removed by the white card correction. Near the end of the processing steps, ratioed data images are divided by the white card matrix to correct for these imperfections (Meyers, 1996).

\section{Pixel Flattening}

White card images do nothing, however, to account for the slight variations in sensitivity for individual pixels across the CCD array. In an effort to force all pixels in an array to have the same sensitivity, two average images are taken with all lenses removed and the array exposed to two different uniform light levels. These images only need to be taken once, since the pixel sensitivity imperfections are inherent to the cameras and are not likely to change with changes to the configuration or alignment of the system. Individual pixel sensitivities (or slopes), which, ideally, should be equal to 1.0, are calculated at each pixel by

$$
\text { slope }_{x, y}=\frac{P 1_{x, y}-P 2_{x, y}}{A v g 1-A v g 2}
$$

where $\mathrm{P} 1_{\mathrm{x}, \mathrm{y}}$ is the gray level value at the $\mathrm{x}, \mathrm{y}$ pixel location in the first image, and $\mathrm{P} 2_{\mathrm{x}, \mathrm{y}}$ is the pixel value at the same location in the second image. Avg1 and Avg2 are the average gray level values for the first and second images, respectively. Data was taken at several (8-10) gray levels with the slopes between any two levels being almost identical. Gray level images of 100 and 200 were chosen to represent the linear response of the CCD array and form the sensitivity correction. The correction is applied by dividing the data image from each camera by the corresponding array of pixel slopes (Meyers, 1992). Figure 4.8 shows an example of $\mathrm{X}$ and $\mathrm{Y}$ cuts through the center of a flat-field image from one of the cameras before and after the pixel sensitivity correction has been applied. The $\mathrm{X}$ and $\mathrm{Y}$ cuts were separated by 5 gray levels, and the two sets are separated by 10 gray level values. Otherwise, the four curves would be practically indistinguishable. The RMS deviations of the $\mathrm{X}$ and $\mathrm{Y}$ cuts before the correction are 1.7 and 0.98 gray levels. After the correction, they are reduced to values of 0.92 and 0.86 , respectively. When viewing false color images of the pixel correction buffers, unique "hot spot" patterns can be seen for each camera in a pattern that is unique to that camera. These patterns are 
identical in shape to features seen in the raw data images, which are removed from the slope images, reaffirming the need for this type of correction.

\section{Smoothing}

The next step in the algorithm is to low-pass filter the image resulting from the steps above. A convolution is performed between a flat $5 \times 5$ kernel and the image, in effect, blurring it. Low-pass filtering reduces the effects of both the CCD readout noise, as well as any laser speckle noise (McKenzie, 1997). Speckle noise is less of a problem with the cw laser used in this research than with a pulsed laser, but low-pass filtering still improves the quality of the images acquired. As a result of testing described below, the modulation transfer function (MTF) of the camera/lens combination was found to be approximately 3 to 5 pixels wide, so a $5 \mathrm{X} 5$ kernel actually causes minimal loss of meaningful velocity information.

An examination of the magnitude of the noise caused by laser speckle has been conducted on two targets. The first was the wheel surface which was painted anodized aluminum, and the second surface was a large piece of poster board. The results in both cases were very similar, so only the results for the wheel are discussed here. Two sets of images, taken while laser light was scattered from the surface of the disk, were acquired and analyzed. The analysis was limited to the examination of the standard deviation of the pixel gray level values over a selected area of the image which was slightly smaller than a rectangle inscribed within the boundary of the disk. The first set of images was taken when the wheel was stationary. This set produced a standard deviation of approximately $20 \%$ of the average gray level. The speckle interference pattern could easily be seen by eye during this test. The second set of data was taken with the wheel rotating slowly ( $20 \mathrm{rpm})$. The RMS variation for this data set was approximately $10 \%$ of the average gray level value, a 50\% reduction over the stationary data. Ideally, though, the speckle pattern would be perfectly correlated between signal and reference cameras, effectively canceling any variation and producing a flat ratio image. When signal and reference camera speckle images were dewarped and overlaid, the speckle noise was reduced to $3 \%$ of the average gray level value. 
Additional speckle testing at different laser power levels was performed to determine the extent to which the speckle noise (as defined by the RMS percentages of the mean pixel value) was independent of scattering intensity. Four intensities were tested with average gray levels ranging from 140 at the highest laser power setting to 30 at 0.6 watts of laser power. The RMS percentage increased with decreasing average intensity, from $17 \%$ at 140 gray levels to $22 \%$ at approximately 30 gray levels. A 5\% change over a $79 \%$ reduction in intensity is negligible, and could possibly be attributed partly to camera effects such as readout noise or pixel bleed.

Another artificial source of intensity variation that the low pass filter helps to correct is the CCD camera itself. In transferring the image from the CCD array to the data bus, there exists readout noise. Readout noise is the partial transfer of one sensor element's charge to a neighboring element. When pixel charge is read from an array, it is done serially; that is, row by row, each charge element in a row moving to the next bin, previously occupied by a neighboring charge, and whole rows moving up until all of the elements are read, one by one. In this bucket brigade of charge transfer, some electrons are left behind and added to the following charge, but it too leaves some electrons behind, and if it leaves behind a number unequal to what it gains, then there will be readout noise. A similar effect can be seen when pixel bleed occurs. For example, when viewing a high contrast image such as a sharp black object on a white background, the transition between black and white should be no more than one pixel wide. However, pixel bleed results from neighboring CCD elements with widely varying charges "leaking" charge to lower potential elements. When combined with readout noise, the resulting transition occurs over a greater number of pixels (Amelio, 1974). Measuring this transition provides information on the effective resolution of the CCD array by characterizing the modulation transfer function (MTF). For the Hitachi cameras used, the MTF was approximately 4-5 pixels wide. An example output from the measurement of a sharp transition from light to dark is shown in iFigure 4.9! This figure illustrates the MTF for the camera/lens combination, where the lens was operated at an f-number of approximately 4.5. The actual MTF for the camera alone is likely to be slightly better. 


\subsubsection{Spatial Correction - Dewarping}

Another averaged image is one of a rectangular reference grid of small dots placed in the plane of measurement which is key to the spatial corrections needed for accurate alignment of the signal and reference camera images. This "dot card" image provides reference points with which dewarping calculations are made.

The need for dewarping is obvious when trying to overlay velocity images to resolve orthogonal velocity components. Resolution of those components necessitates measuring velocity from different directions, resulting in perspective warping. However, even though both signal and reference cameras within a DGV component system are viewing the same area through a beamsplitter, dewarping is also needed for these images to correct for imperfect pixel-to-pixel alignment (Meyers, 1992). Since the measurements are taken in a plane, the dominant distorting effect is a perspective warping (keystone effect) originating from non-perpendicular imaging. If this were the only effect, the correction would be a simple linear transformation applied to the whole image at once. However, there is also significant warping due to irregular optics; specifically, the beamsplitter is a thin piece of glass 4 inches in diameter which bends easily within the mounting hardware, producing distortions that are most noticeable in the reference image. Vertical lines cannot be made to simultaneously appear vertical in this image.

The dewarping process begins with the acquisition of a dot card image by each camera, as described above, which provides an array of discrete reference points with which to align the images. Each dot card image is then masked by passing the result of a standard edge-finding (Sobel) filter through a threshold function, and the dot center locations are found using blob centroid analysis routines provided by the frame grabber manufacturer. The centroid coordinates are calculated by weighting the gray level of each pixel identified by the threshold function as part of a dot, then finding the second moment for the grouping. The centroid coordinates are then stored for each image.

Next, a grid is generated which marks the location of the dots in the dewarped image. The numbers of rows and columns of dots visible in the distorted image are counted, and the grid coordinates are calculated in such a way as to fill the entire dewarped image with that number of equally spaced points. This has been done so that 
the warped image is always stretched when dewarped, and pixels referenced in the warped image are guaranteed to be defined within the image area, leaving no holes or overlap. Consequently, the resulting dewarped images will thus almost always have slightly different $\mathrm{X}$ and $\mathrm{Y}$ scale factors; however, this distortion has been minimized through the use of a grid pattern that has the same aspect ratio as the CCD array.

The centroids of the dots in the warped image are sorted in the $\mathrm{x}$-coordinate by Shell's method (Press, et. al., 1992). Next, the three closest dots to the upper left corner of the image $(0,0)$ have been found using a standard two dimensional distance formula. The upper left most dot is the dot with the minimum distance to the origin. The dot which is closer in the $\mathrm{X}$ direction than in the $\mathrm{Y}$ direction is the second dot in the first column, and the last dot in the group of three identified is the second dot in the first row. These three dots are then used to predict the location of dots in the first row and column, which, in turn, are used to predict the second row and column, and so on. In this manner, the dots are assured to be sorted into rows as they are viewed on the dot card, provided the warping exists at a reasonable level as can be expected in a planar imaging system such as DGV.

Next, two floating point buffers which are the same size as a data image, are filled with $\mathrm{X}$ and $\mathrm{Y}$ coordinates. The $\mathrm{X}$ and $\mathrm{Y}$ coordinates specify exactly where, in the warped image, the gray level value for every pixel in the dewarped image came from. The values that are placed in these buffers are calculated by first finding where each pixel is as a percentage of distance from the four pixels that surround it. Then, those percentages are used to find the $\mathrm{X}$ and $\mathrm{Y}$ coordinates of the location that is in the same position relative to the four surrounding dots as they appeared in the warped dot card image (Wolberg, 1990). Figure 4.10' 'gives a visual representation of this process.

When this is done, there exist two floating point arrays for each camera; one containing X pixel coordinates, the other Y. To dewarp a warped data image, first the appropriate $\mathrm{X}$ and $\mathrm{Y}$ buffers are loaded into memory. Then, pixel by pixel, the $\mathrm{X}$ and $\mathrm{Y}$ coordinates are read from the buffers, and if both coordinates are whole numbers, then the gray level from that location in the warped image is placed in the same location that the coordinates were read from, in a separate, blank image buffer. However, the $\mathrm{X}$ and $\mathrm{Y}$ 
coordinates are not likely to be whole numbers, so the interpolated values from the four closest pixels to each coordinate normally must be calculated for each dewarped pixel. Here, the percentage distance from the four neighboring pixels can be read using the decimal portion of the $\mathrm{X}$ and $\mathrm{Y}$ coordinates since pixels are enumerated as a whole number grid. Equations used for the bilinear interpolation are given in Equation 5.

$$
\begin{aligned}
& A=P_{x, y}+u^{\prime}\left(P_{x+1, y}-P_{x, y}\right) \\
& B=P_{x, y+1}+u^{\prime}\left(P_{x+1, y+1}-P_{x, y+1}\right) \\
& D G L=A+v^{\prime}(B-A)
\end{aligned}
$$

In these equations, $\mathrm{P}_{\mathrm{x}, \mathrm{y}}$ is the gray level at pixel location (x,y) in the warped image, $\mathrm{u}$ ' and $\mathrm{v}^{\prime}$ are the $\mathrm{X}$ and $\mathrm{Y}$ percentages defining location between four points, and DGL is the dewarped gray level.

Two tests were conducted to test the accuracy of the dewarping algorithm. The first test, which was more of a test for dewarping repeatability, involved acquiring 30 sets of four dot card images. The dewarping coefficients were found for each set of images using the algorithm described above. During this process, the centroids for all reference dots in the original, warped images were recorded. The original dot centroid locations were then dewarped and their locations in the dewarped buffer were recorded. The standard deviation of all 120 sets of dewarped centroid locations from the desired equally spaced grid was found. The standard deviations were approximately \pm 0.00001 pixels, which is on the order of the floating point accuracy with which the centroid locations were stored. The second test was conducted to quantitatively describe the accuracy of the dewarping of a single image. The normal procedure for dot card image acquisition and processing was followed for a dot card which had approximately 10:1 dot spacing and a 4:3 aspect ratio (2" X 2.7"). The dot card was then moved in the plane of the card using the 3-axis computer controlled traverse. The card was moved in both the vertical and horizontal directions an amount equal to half the dot spacing, and the normal acquisition and processing procedure was repeated. The centroid locations for both dot card images were then dewarped using the coefficients calculated from the dot card image taken before the movement. The $\mathrm{X}$ and $\mathrm{Y}$ difference between the pre- and post-move dewarped coordinates of the dot centroids was then calculated. The distance moved was made to be 
half of the dot spacing in an attempt to represent the dewarping of the pixels that were farthest away from the dot reference points, and therefore, most likely to have the largest associated errors. Table 1 shows the standard deviations of the differences in $\mathrm{X}$ and $\mathrm{Y}$ coordinates for the pre- and post-move dewarped images for all four cameras.

Table 1. Standard deviations of dot centroids (in pixels)

\begin{tabular}{|c|c|c|c|c|}
\hline & Camera A & Camera B & Camera C & Camera D \\
\hline $\mathrm{X}$ & 0.25 & 0.27 & 0.34 & 0.28 \\
\hline $\mathrm{Y}$ & 0.19 & 0.20 & 0.18 & 0.18 \\
\hline & \multicolumn{2}{|c|}{ A - B } & \multicolumn{2}{c|}{ C - D } \\
\hline $\mathrm{X}$ & \multicolumn{2}{|c|}{0.34} & \multicolumn{2}{c|}{0.43} \\
\hline $\mathrm{Y}$ & \multicolumn{2}{|c|}{0.28} & \multicolumn{2}{c|}{0.24} \\
\hline
\end{tabular}

The bottom half of Table 1 gives the standard deviations of the dewarped postmove dot centroids between cameras of the same velocity component, which is a better measure of the overlay accuracy. An example of the overlay accuracy between signal and reference cameras for both velocity components is shown in Figure 4.11: The $X$ coordinate standard deviations in Table 1 are consistently higher than that of the $\mathrm{Y}$ coordinate due to the type of warping caused by the geometry of the system tested. All cameras were placed in the same plane; therefore, the primary warping is due to perspective effects which have a greater influence on the apparent width of the image. The dot card used for this test was approximately 2 " high and $2 \frac{1}{2}$ " wide. The test has been repeated with another dot card approximately 6 times taller and wider. The standard deviations for the larger dot card are approximately half the values of those shown above, much closer to the 0.3 pixel accuracy quoted by McKenzie (1997). The increased fnumber that was a result of a smaller focal length needed to image the larger dot card is thought to be responsible for an improvement in the accuracy and consistency with which the algorithm calculates the centroid location of each dot. This would lead, in part, to a smaller variation in dewarped centroid location.

\section{$\underline{4.3 .3 \text { Division }}$}

The dewarped signal and reference images for each component are checked for zero or extremely low gray level values, which are replaced with a value of -1 for the reference images, and 20 for the signal images. This produces an out-of-range ratio value 
when passed through the curve-fit equations if either one or both of the images has lowvalued pixels. The signal and reference images are then divided, producing, where sufficient signal exists, a ratio "image" containing values which are proportional to velocity. The ratio for each pixel is checked for out of range values (produced in the step above) before being passed through the curve-fit of the appropriate cell calibration data by checking the ratio value against the upper and lower ratio limit of the calibration curves. If a pixel has an out of range ratio value, it is marked and set equal to a mid-range value so that no numeric errors occur during the calculation. After all calculations have been completed, the marked pixels are set to zero frequency shift which results in a zero velocity reading in the final velocity image.

The frequency found from dividing the signal and reference voltages from the reference system photodiodes and passing the resulting ratio through the reference curvefit is subtracted at this point from both relative frequency arrays of the two components. The goal of subtracting the reference system frequency is to eliminate the velocity change that would be measured as a result of laser frequency drift. The effectiveness of this reference system will be discussed in a later section. At this point, there exist two Doppler frequency "images" which are used in Equation 1 to produce two velocity images. Velocity images are actually floating point buffers which can not be displayed normally, but when scaled from 0 to 255 , can be viewed as grayscale or color images. The combination of system geometry and laser wavelength in Equation 1 yields a number that is a constant for each component for a particular test setup. This number is called the component sensitivity because it quantifies the measured Doppler frequency shift per $(\mathrm{m} / \mathrm{sec})$ of velocity along the sensing direction. It has units of $\mathrm{MHz} /(\mathrm{m} / \mathrm{sec})$, with typical values ranging from $2-3 \mathrm{MHz} /(\mathrm{m} / \mathrm{sec})$. The frequency images are simply divided by the corresponding sensitivity to produce velocity images as the final step in the data reduction process. 


\section{Chapter 5: VELOCITY MEASUREMENT EXPERIMENTS}

\subsection{Rotating Wheel}

Two experiments were used to evaluate the accuracy of both the point and planar velocimetry methods. The first and most basic experiment involved the measurement of the velocity of a rotating wheel. A constant RPM wheel provides a good velocity standard to compare with measurements. The wheel used was a black anodized aluminum disk approximately twelve inches in diameter, driven by a speed controlled DC motor. The wheel was tilted at a shallow angle to the laser beam, and the beam was spread into a cone with a small, $9 \mathrm{~mm}$ diameter, f1 lens, such that the entire surface of the disk was illuminated. The black anodized surface of the disk was painted white to create more scattered light, and therefore a higher signal level for the receiving optics. For the planar system, the wheel was tested at maximum RPM only. Variation of the wheel speed or traversing of the wheel itself was not needed as it was in the point system, because a single image shows the entire range of in-plane velocities along a vertical diameter. The RPM of the motor at the maximum setting on the speed controller was measured by aiming a laser pointer from in front of the wheel, into a photodiode mounted just behind the spinning disk. Thin strips of heavy stock paper were taped to the edge of the wheel, protruding just enough to break the beam of the laser pointer to the photodetector. The output of the photodetector was then viewed on an oscilloscope where the period between the strips was read. The angular velocity was calculated and was used to determine tangential velocity when multiplied by the radius. The controller setting which produced the measured RPM was found by measuring the voltage on one side of a voltage dividing potentiometer within the controller, providing a much more reliable way to consistently set the speed than to try and return to the same mark on the dial each time by eye. The entire wheel was imaged using the setup shown in'Figure $3.3 \vdots$ 5.2 Pipe Flow

The second experiment was the measurement of fully developed, turbulent pipe

and turbulent jet flow. Configuration and geometry of the test is shown in Figure 3 1.5" diameter copper pipe, $90 "$ long $(\mathrm{L} / \mathrm{D}=60)$ was attached to a high speed centrifugal 
type blower. Reynolds number for the pipe was approximately 100,000 based on diameter. Flow seeding was added to the intake of the blower by a Rosco fog machine which vaporized a propolyene glycol based fluid into "smoke" particles on the order of 1 to $10 \mu \mathrm{m}$ in diameter. A large volume container was placed in-line between the fog machine and the blower intake to minimize the pulsating effect with which the machine dispensed the fog. Downstream of the exit of the pipe, a 3:1 reducing cone connected to exhaust ducts was used to remove the fog from the pipe flow out of the building. A laser light sheet formed by the $\mathrm{x}-\mathrm{y}$ scanner head was placed at various positions at the exit plane as well as multiple diameters downstream, and the fog particles scattered the laser light into the receiving optics of the DGV velocity measuring systems. The speed of the flow was governed by the blower RPM which was controlled by a variable voltage AC transformer; a maximum speed of approximately $40 \mathrm{~m} / \mathrm{s}$ was set by limitations in removing the smoke from the lab. The settings on the transformer were calibrated to pipe velocity with a pitot-static probe. 


\section{Chapter 6: RESULTS}

\section{$\underline{6.1 \text { Wheel Results }}$}

Analysis of the wheel velocity data is presented as RMS variations and percent error of the total velocity range (since the wheel velocity distribution is linear). The RMS error is the standard deviation of the velocity from a straight line fit through the data, not the deviation from the known velocity. It is a good descriptor of the minimum resolvable velocity of the instrument. Since the wheel velocity distribution (in the plane of the instruments) is linear for any vertical cut down a wheel image, the RMS error from a linear fit is also a good indicator of the error level resulting from a combination of several different sources. Camera noise, 8-bit digitization, truncation in data processing, various sources of environmental electromagnetic radiation, and non-uniformity in the wheel surface all contribute to the RMS error.

Percent error of the total velocity range is an indication of the overall accuracy of the system. Contributors to this type of error tend to be more systematic, such as the nonrepeatability of the iodine cell calibrations, camera and photodetector dark voltage bias, measurement errors in the system geometry, and the accuracy to which the wheel speed (or pipe/jet flow) can be determined by an alternate measurement device.

Four sets of 30 images of the surface of the wheel were acquired while the wheel was rotating at maximum RPM. The individual velocity images found along the sensitivity vector for each velocity component are treated as projections of the actual velocity vector in the sensitivity directions of the respective components (See equation 1

and Figure 3.2j. As a result, each component under-estimates the value of the velocity vector by measuring a quantity that is the cosine component of the angle between the velocity vector and the measurement angle. If the direction of the velocity vector can be assumed, as can safely be done in the case of a rotating wheel, each component can be made to measure the velocity in that direction.

The average of 30 images taken in one 15 second set $\left(1 / 60^{\text {th }}\right.$ of a second exposure, each) are shown for each component in 'Figures 6.1 and 6.2 .2 . Both measuring components are reduced in the direction of the known velocity vector (parallel to the surface of the wheel), producing velocity images which show the comparative accuracy of each velocity 
component very well. 'Figure 6.1 is the velocity image from component \#1, which shows more non-uniformity due to a large measurement angle (with respect to the velocity vector) of $66.5^{\circ}$. Any measurement made at that angle was multiplied by $1 / \cos \left(66.5^{\circ}\right)$ or 2.5. Component \#2, shown in Figure 6.2, has much less variation giving it an overall smoother appearance. Referring to Figures 3.2 and 3.3, it can be seen that the measurement angle for component \#2 is a much smaller $35.5^{\circ}$.

Data read from horizontal and vertical cuts through the center of these images and trimmed at the edges of the wheel are shown in Figures 6.3 and 6.4 Cuts were frequently used to more closely evaluate the quantitative accuracy of the measuring components. Table 2 shows the standard deviations and velocity ranges of the horizontal (X) and vertical (Y) cuts of the average velocity images. The total velocity range for the rotating wheel was $58.7 \mathrm{~m} / \mathrm{s}$. In the $\sigma \mathrm{Y}$ case, the standard deviation was calculated as the variation from the linear fit. These data highlight the dependence of instrument precision on the system geometry because RMS values from component \#2 are consistently double those from component \#1, and the velocity magnitude errors are smaller by a factor of 10 .

Table 2. Standard deviations $(\sigma)$ and measurement accuracies of average image $X$ and $Y$ cuts $(\mathrm{m} / \mathrm{s})$ for components 1 and 2

\begin{tabular}{|c|c|c|c|c|c|c|}
\hline Data Set \# & $\boldsymbol{\sigma X 1}$ & $\boldsymbol{\sigma X 2}$ & $\boldsymbol{\sigma} \mathbf{Y 1}$ & $\boldsymbol{\sigma} \mathbf{Y} 2$ & $\Delta \mathbf{V}_{\mathbf{1}}(\boldsymbol{\%}$ error) & $\Delta \mathbf{V}_{\mathbf{2}}$ (\%error) \\
\hline 1 & 2.96 & 1.30 & 2.19 & 1.19 & $47.6(18.9 \%)$ & $59.2(0.9 \%)$ \\
\hline 2 & 3.25 & 1.27 & 2.17 & 1.12 & $48.2(17.9 \%)$ & $59.3(1.0 \%)$ \\
\hline 3 & 2.63 & 0.91 & 2.28 & 1.22 & $51.1(12.9 \%)$ & $57.5(2.0 \%)$ \\
\hline 4 & 2.85 & 0.90 & 2.45 & 1.25 & $50.9(13.3 \%)$ & $57.8(1.5 \%)$ \\
\hline
\end{tabular}

The data were reduced a second way by calculating the velocity in two orthogonal directions, which is by far the more practical case since no assumptions are made about the direction of the flow being tested. In the rotating wheel experiment, the orthogonal directions were chosen to be normal and tangential to the surface of the wheel (see Appendix B).

Two-component rotating wheel DGV data is presented in Figures 6.5 and 6.6. 'Figure 6.5 'is the velocity image of the component parallel to the surface of the wheel, and Figure 6.6 ; is the component normal. Both images are a combination of the results of the two measurement systems. Cuts through these images are shown in'Figures 6.7 and 6.8 . The range of velocities on the wheel are linearly distributed along any vertical cut with 
the maximum range occurring at the horizontal center of the wheel. The maximum and minimum velocities for a vertical center cut are $29.35 \mathrm{~m} / \mathrm{s}$ and $-29.35 \mathrm{~m} / \mathrm{s}$ for a total range of $58.7 \mathrm{~m} / \mathrm{s}$. The DGV measurement shown has a range of $61.6 \mathrm{~m} / \mathrm{s}$ for a $5 \%$ error. The normal velocity component is known to be zero since the wheel never moves out of plane, however, the measured velocity in'Figure 6.8 shows wide variations of up to \pm 10 $\mathrm{m} / \mathrm{s}$ with a maximum standard deviation of $3.8 \mathrm{~m} / \mathrm{s}$. This is due to the fact that the component providing the most velocity information in the normal direction is the inherently noisier component \#1. The RMS values and tangential velocity ranges for all four data runs are shown in Table 3.

Table 3. Standard deviations $(\sigma)$ of orthogonal velocity components, and tangential component measurement accuracies $(\mathrm{m} / \mathrm{s})$

\begin{tabular}{|c|c|c|c|c|c|}
\hline Data Set \# & $\boldsymbol{\sigma} \mathbf{X}_{\mathbf{T}}$ & $\boldsymbol{\sigma} \mathbf{X}_{\mathbf{N}}$ & $\boldsymbol{\sigma} \mathbf{Y}_{\mathbf{T}}$ & $\boldsymbol{\sigma} \mathbf{Y}_{\mathbf{N}}$ & $\Delta \mathbf{V}_{\mathbf{T}}(\boldsymbol{\%}$ error $)$ \\
\hline 1 & 1.30 & 3.10 & 1.67 & 3.12 & $63.4(8.0 \%)$ \\
\hline 2 & 1.24 & 3.50 & 1.58 & 3.04 & $63.5(8.1 \%)$ \\
\hline 3 & 1.43 & 3.50 & 1.62 & 3.27 & $61.6(4.9 \%)$ \\
\hline 4 & 1.49 & 3.89 & 1.77 & 3.58 & $62.0(5.6 \%)$ \\
\hline
\end{tabular}

Data were obtained for the same rotating wheel by the two-component Point Doppler Velocimetry (PDV) system (Kuhlman, et. al., 1997) developed prior to the planar system described in this work. The RMS deviations of that data, reduced in orthogonal directions, averaged approximately $0.5 \mathrm{~m} / \mathrm{s}$, while the percent error in velocity range was less than $2 \%$. The best data acquired with the planar system shows an approximate factor of 3 increase in error over a point system that had 257 times greater resolution (8-bit DGV compared to 16-bit PDV). Also, each rotating wheel velocity measurement for the PDV system was the average of several hundred data points collected over times on the order of seconds. In the same time scale, the DGV system might acquire 5 individual data sets.

The effect of the white card correction as discussed in Section 4.3.1, is shown in 'Figures 6.9 and 6.10.' The image shown in'Figure 6.9 'contains the same data as ífigure 6.6, but reduced without the white card correction. The circular patterns visible in the image are interference fringes present in only the signal camera of each measurement component. The fringes are a result of an interferometer-like setup of the iodine cell end windows. This irregularity illustrates the need for laser light illumination of the white 
card, because had the white card been illuminated with white light, the interference fringes would not be present in the white card images nor corrected for in the data images. The range of variation of the component \#1 white card ratio along vertical and horizontal cuts is 1.09 to 1.02 . When the ratio of the data images is divided by the white card ratio, this $7 \%$ variation causes a \pm 0.02 change in data ratio. Assuming the data ratio is approximately 0.57 , the iodine cell absorption profiles translate \pm 0.02 changes in ratio to $\pm 9.7 \mathrm{MHz}$ of frequency change. Using the geometry of component \#1, because it is the main contributor to the velocity image shown in 'Figure 6.9 the white card variation produces a $\pm 8.8 \mathrm{~m} / \mathrm{s}$ change in velocity. Ideally, this correction would be of equal and opposite magnitude as the data image errors. In practice, though, while it has roughly the same variations as the data errors, the error in the measured data is not reduced by an amount equal to the change in the white card because the white card contains errors of its own. Gross deviations (such as the interference pattern) in the data images are greatly reduced, but smaller variations in relatively featureless areas of the data images are not as well correlated with the white card ratio variations, and thus, do not produce the same level of correction. Perhaps this white card correction can be further improved.

\subsection{Turbulent Pipe/Jet Flow Results}

Using the geometry shown in:Figure 3.4, a large set of data measuring the flow exiting the 60 diameter pipe apparatus was taken. Representative selections from this data set will be subsequently discussed. In all velocity images shown for seeded flow, the pictured result is the average of 30 individual images acquired over approximately a 10 second time period, and both components have been reduced along the axial direction of the pipe using the same dot card warping correction for all data sets. The Reynolds number for the pipe flow was approximately 100,000 based on diameter. Also, the approximate diameter and position of the pipe has been shown by a black circle in each image.

Several improvements to the system had to be made before obtaining these data sets. A coaxial duct was installed to deliver low speed smoke particles to the entrainment area around the exit of the pipe, with the object of lowering the light intensity gradients in the mixing layer. A second smoke generator injected a vapor mist into a large volume 
container which then exhausted the particles through a four-inch dryer duct which was placed around the end portion of the pipe. Data acquired previous to this modification exhibited large errors in the mixing layer, where particle concentration decreased very quickly. In areas of sufficient signal, a correction needed to be made to offset a random velocity bias of unknown origin. It is the purpose of the reference frequency monitoring system to compensate for all velocity bias due to laser frequency change. However, correlations of the reference system ratio to that of the velocity measuring systems were less accurate than expected. A more direct approach was taken to correct for any velocity offset bias by providing a laser illuminated, non-moving object in the field of view of each component. An uncoated 2" piece of Pyrex ${ }^{\circledR}$ was placed in the beam path, and the approximately $8 \%$ reflection from the first surface of the glass was focused into a $300 \mu \mathrm{m}$ fiber optic cable. The fiber optic was used to illuminate a small, rectangular piece of paper which served as the "zero tab". The velocity of this tab, as measured by the two DGV components, was subtracted from every velocity image, including all results presented here. An earlier attempt using a separate defocused zero tab for each component was not as successful as using a single tab in field of view of both components.

Problems with obtaining a uniform and properly corrective white card have been addressed by first identifying the location of the interference rings that were present in every camera signal image. The iodine cell, which caused the fringes as discussed earlier, was slightly rotated, enough to move the fringes away from the center of the image. Two different white cards were then taken. The first was actually a set of two white cards, one for each component. A piece of poster board was illuminated very close to each component such that it appeared out of focus. While acquiring white card images, both the poster board and a thin plastic film optical diffuser were in constant motion to help create a smooth image free of speckle and numerous interference fringes produced by the $9 \mathrm{~mm}$ focusing lens at the end of the fiber optic. The second white card was placed in view of both components near the measuring plane and illuminated in a similar manner. This card resulted in a correction that was a more successful than the first. 
Figures 6.11 and 6.12 contain velocity images from component \#2 which is looking back on the measuring volume from downstream (see Figure 3.4ij. Because of this angle, component $\# 2$ is able to clearly view measurements of turbulent pipe flow taken at the pipe exit, while component \#1 is not. 'Figure 6.11 ; taken 0.2 inches from the pipe exit, shows the average velocity distribution of fully developed turbulent pipe flow as measured by component \#2. The spots on the left side of the image are specular reflections of the scattered laser light from the lip of the copper pipe. After traversing the pipe apparatus 1.5 inches away ( 1 diameter) from the laser sheet, iFigure 6.12', was obtained. At this position, component \#1 is just able to view the entire measuring volume. However, for this data set, some of the pixels in the center of the image received too much signal and were saturated, producing erroneous velocity data. Images that contained saturated data have not been included in these results. Significant mixing and shear layer growth can be seen in Figure 6.12ijust 1 diameter downstream of the exit. Horizontal and vertical cuts through the center of these images are shown in:Figures 6.13, and 6.14 ; Figure 6.13 approaches the classic profile of fully developed turbulent pipe flow except for approximately $3 \mathrm{~m} / \mathrm{s}$ fluctuations and a few minor anomalies (for example, on the left side of the image) that can be attributed to secondary scattering and reflections from the illuminated edge of the copper pipe. iFigure 6.14'shows a more rounded profile which is to be expected due to the shear layer growth at the increased downstream location. Approximately 200 pixels span this profile, for a 100 pixels/inch spatial resolution. Both the horizontal and vertical cuts have the same overall shape in both figures, as they should since the pipe flow is axisymmetric. The peak magnitudes of all of the velocity plots show an inconsistency that is entirely due to the not-completelyperfected method of setting up and acquiring an accurate zero tab. The peak centerline velocity value as measured by a pitot probe at the exit of the pipe is $42 \mathrm{~m} / \mathrm{s}$ for both data sets.

A two-component measurement 1 diameter from the exit is shown in'Figures 6.15 and 6.16 .9 The velocity fluctuations on the right side of:Figure 6.15 are believed to be due to secondary scattering from core flow particles. A similar phenomena can be seen in Figure 6.16, but on the left side and with reduced intensity. Referring to Figure 3.4 , 
component \#1 must view the right side of the measuring volume through a dense stream of seed particles. Likewise, imaging of the left side by component \#2 must take place through a similar volume of particles, albeit less dense. This may also account for the slight imbalance shown in the horizontal and vertical cuts (Figures 6.17 and 6.18) through these images. That is, a slightly higher velocity is measured in regions of higher secondary scattering. Noise in these cuts is visually estimated to be approximately $5 \mathrm{~m} / \mathrm{s}$ peak to peak, or less than $2 \mathrm{~m} / \mathrm{s}$ RMS. This is comparable to the noise in the wheel data results.

Measurements taken 2 diameters from the exit of the pipe are shown in Figures! 6.19 and 6.20. In these images, the middle velocity range (in green) has spread to cover a greater portion of the radius. The core velocity is less well defined in these images. Quantitative support of these observations can be seen in'Figures 6.21 and 6.22 . Figure 6.23 shows even more mixing as the jet has spread to approximately 2 diameters in width 6 inches (4 diameters) downstream. The measurement from component \#1 was again saturated, and has not been shown here. The profile shown in Figure 6.24'has a smooth Gaussian shape characteristic of a jet profile. Also, the edges of the image show more velocity fluctuations because of both higher turbulence intensities in this region and a lower probability of particle illumination. Once again, the inconsistency of the zero tab can be seen in'Figure 6.24.1 At 4 diameters, the centerline velocity should be slightly less than the $42 \mathrm{~m} / \mathrm{s}$ measured at the pipe exit. If the velocity data near the edges of the image are assumed to be close to zero, iFigure 6.24 's shows a maximum velocity of $35-40 \mathrm{~m} / \mathrm{s}$, whereas use of the zero tab indicates $47 \mathrm{~m} / \mathrm{s}$. This would be closer to the $40 \mathrm{~m} / \mathrm{s}$ centerline velocity measured by the pitot probe.

Measurements taken at 6 diameters (9 inches) downstream are shown for both components in Figures 6.25 and 6.26 . The width of the maximum velocity region seen in the images has been reduced to a fraction of the pipe diameter, indicating a steeper profile, while the mixing and entrainment has increased the local jet diameter to be between 2 and 3 pipe diameters. In'Figure 6.26 ,'striations can be seen in the top and bottom of the image. These are likely due to several 8-bit truncations inherent in the image processing procedure. 'Figures 6.27 and 6.28 ishow good symmetry, although they 
are quite noisy (maximum deviations of 6-12 m/s), especially towards the edges of the flow.

Pitot probe data were taken at the pipe exit and 1 diameter downstream to validate the DGV data. 'Figure 6.29 shows horizontal and vertical cuts through the exit velocity image ('Figure 6.11) overlaid with pitot probe data taken at the same location. Only the offset (approximately $5 \mathrm{~m} / \mathrm{s}$ ) of the DGV data was modified to create the good fit displayed in this figure. Similarly, the offset of cuts through the 1 diameter velocity image were changed to create the overlay in Figure 6.30.i The repeatability of the pitot data is approximately $3-4 \%$ of the maximum velocity. The peak profile velocity was found to be extremely sensitive to small changes in the position of the ducting that delivers the smoke to the blower inlet. The positioning of the duct was bound to change from day to day due to both regular movement around the lab space, as well as operation of the blower itself. Nevertheless, the pitot probe data validates the goodness of the shape of these two profiles and reinforces the self-consistency of the flow velocity measurements.

Images of RMS fluctuations of the jet at 4 diameters downstream provide a limited view of general turbulence characteristics of the jet as indicated by the DGV system results. Figure 6.31; is the RMS of 30 velocity images and shows the characteristics of turbulent jet flow; the center of the jet having a much lower RMS than the edges where larger scaled turbulence is known to exist. Figure 6.32 is the RMS image (Figure 6.31) divided by the mean velocity image ('Figure 6.23 )', pixel by pixel. This image gives some indication of either the noise to signal ratio in areas where the actual flow turbulence is low, or the flow turbulence intensity where the actual flow turbulence level is high. 'Figure 6.33 and 6.34 ' are cuts through the above mentioned RMS images. 'Figure 6.33 'has two interesting features at approximately $\pm 0.4 \mathrm{r} / \mathrm{D}$. It is possible that the two spikes in the RMS plot at these locations identify an increased turbulence value in the shear layer. It is also possible that the spikes are simply noise, but because they occur in both the horizontal and vertical cuts, it is more likely to be a flow feature. Figure 6.34 indicates less than a 10\% RMS velocity fluctuation at the center of the jet. This is slightly lower than the approximately 10-20\% (Kuhlman, 1994) expected along 
the centerline for jet flow. One likely cause for the low RMS values is the long (when compared to estimates of the large eddy time scale of $5 \mathrm{~ms}$ ) exposure time of $1 / 60$ of a second and subsequent averaging by the CCD cameras. Also, 30 images may not be a large enough sample size for turbulence quantities to converge. However, preliminary hot-wire data acquired for this flow suggests an agreement in the turbulence intensities measured at the center of the pipe, 4 diameters downstream. The data also suggests that the self-preserving region characteristic of downstream jet flow takes longer to develop in this turbulent jet. It is not possible that the DGV system measured the small scale turbulence of this flow, which the hot-wire could readily resolve. Therefore, it may be merely a coincidence that the DGV data agrees with the centerline turbulence intensity as measured by the hot-wire. Investigation of this matter is ongoing. 


\section{Chapter 7: CONCLUSIONS \& RECOMMENDATIONS}

A two-component Doppler Global Velocimeter (DGV) system was developed and tested. Techniques for calibrating and acquiring data with the instrument were developed and refined. Some of the more basic principles of this system were tested with a two component Point Doppler Velocimeter (PDV) prior to the construction of the DGV system. The bulk of the experience gained from the PDV system was in the area of iodine cell behavior and calibration procedures.

The transition from successful PDV measurements to successful DGV measurements was not easy. One of the biggest obstacles was the large amount of detailed image processing needed with a planar system. Software was written to start with raw intensity images, apply corrections to them, and calculate the measured velocity. Velocity offsets not compensated for by the reference system continue to plague both PDV and DGV measurements. However, DGV localized velocities with the current system are clearly in correct proportion to other areas in the same velocity image.

Numerous procedures were developed to try and improve the quality of the DGV measurements. Polarizing film filtered all depolarized scattered light collected by the DGV optics to compensate for the polarization dependence of the beamsplitters used; however, the vast majority of light scattered from the seeded flow was still polarized. The polarization film had little effect other than a reduction in signal strength.

Conditioning and regulation of the seed particle density caused a reduction in secondary scattering levels. A large volume container placed in line between the smoke generator and the flow effectively filtered short time-scale fluctuations of the smoke intensity. With this modification, it was easier to regulate the concentration of seed particles over the time period of a test run so that the overall smoke particle intensity could be reduced, minimizing secondary scattering.

The procedure for non-homogenous intensity corrections (white card or flat field) is not yet perfected. Laser illumination of the white card is essential; however, coherent light does not evenly illuminate an area very well. Various forms of interference and non-uniformity prevented the acquisition of a truly corrective white card. Slight 
adjustment of the iodine cell yaw angle aided the acquisition of a homogeneous white card by moving interference fringes set up by the cell to a less critical area in the measurement image. Sealed, vapor-only iodine cells manufactured to less stringent tolerances may help ease the detrimental effects of cell-generated fringes. Some improvement in uniformity may be gained by illumination of the white card by means of secondary reflection. In addition, diffusion of the laser light coupled with movement of the white card while averaging a large number of images provided the best results for the white card correction.

The addition of coaxial smoke generation helped the accuracy of seeded flow experiments near the edge of the velocity region, but this smoke needs to be delivered to the exit plane in a more uniform, less turbulent manner. Great care was taken to insure that the dot card was imaged in the same plane as the laser sheet. The testing of slight out-of-plane dot card positions (as small as the width of the laser beam) produced significant measurement errors.

Much room exists for improvement in the image processing software. Loss of information due to truncation at various points in the software could be avoided by rewriting some portions of the code. Obtaining higher resolution data images from 12 or 16-bit cameras would help the loss of data precision as well. The current software was written without speed optimization in mind. A significant reduction in processing time could be gained with some attention given to this area.

Recommendations on correcting the inconsistent velocity offset are not as obvious or plentiful, yet this is currently the biggest obstacle to having a fully developed instrument. There exists a poor correlation between the reference system ratio, which is essentially an offset measuring component, and the velocity measuring systems. For an accurate measurement of offset, this cannot be. The zero tab is essentially an auxiliary offset measuring device that works reasonably well when placed in the plane of measurement. Perhaps more uniform illumination of the zero tab from a stronger laser light source could ease this restriction.

The best accuracy documented by this system was less than $2 \%$ error on both the RMS variation from a precise measurement and the total range velocity error on a rotating 
wheel with maximum tip speed of $\pm 29.4 \mathrm{~m} / \mathrm{s}$, which is better accuracy than most published results to date. For pipe/jet flow measurements, minimum RMS noise values were estimated to be comparable to that of the wheel data $(1-3 \mathrm{~m} / \mathrm{s})$. The pipe/jet average velocity data also agrees well with pitot probe measurements of the same flow. The maximum velocity offset that was subtracted from the data to match the pitot and DGV profiles was approximately $5 \mathrm{~m} / \mathrm{s}$, which is on the order of the repeatability of the pitot probe measurements $(2 \mathrm{~m} / \mathrm{s})$. RMS measurements made with the DGV system 4 diameters downstream of the pipe exit agree with hot wire measurements in the center of the flow. The validity of the turbulence intensities as measured by the DGV system is still in question, since there is an inherent $1 / 60 \mathrm{sec}$. averaging in the camera based system. Continuing research on the white card correction is a high priority. The white card and zero velocity tab are seen as the biggest limiting factors to obtaining more accurate and less noisy results. 


\section{REFERENCES}

Ainsworth, R.W., and Thorpe, S.J., "The Development of a Doppler Global Velocimeter for Transonic Turbine Applications," International Gas Turbine and Aeroengine Congress and Exposition, The Hague, Netherlands, June13-16, 1994.

Ainsworth, R.J., Thorpe, S.J., and Manners, R.J., "New Approach to Flow-Field Measurement - a View of Doppler Global Velocimetry Techniques," International Journal of Heat and Fluid Flow, Vol. 18, No. 1, pp. 116-130, Feb., 1997.

Amelio, G.F., "Charged-Coupled Devices," Scientific American, pp. 232-247, February 1974.

Arnette, S.A., Samimy, M., Elliot, G.S., " The Effect of Expansion on Large Scale Structure Evolution in a Compressible Turbulent Boundary Layer," AIAA Paper 94-2228, 25th AIAA Fluid Dynamics Conference, June 20-23, 1994.

Beutner, T.J. and Baust, H.D., "Recent Developments in Doppler Global Velocimetry," Paper No. 8 of the AGARD Fluid Dynamics Panel $81^{\text {st }}$ Meeting and Symposium on Advanced Aerodynamic Measurement Technology, Seattle, WA, Sept. 22-25, 1997.

Bloom, S.H., Kremer, R., Searcy, P.A., Rivers, M., Menders, J., and Korevaar, E., "LongRange, Noncoherent Laser Doppler Velocimeter," Optics Letters-Vol. 16, No. 22, pp. 1794-1796. Nov. 15, 1991.

Bloom, S.H., Searcy, P.A., Choi, K., Kremer, R., and Korevaar, E., "Helicopter Plume Detection Using an Ultranarrow-Band Noncoherent Laser Doppler Velocimeter," Optics Letters-Vol. 18, No. 3, pp. 244-246. Feb. 1, 1993.

Bruun, H.H., Hot-Wire Anemometry: Principles and Signal Analysis, Oxford University Press, New York, NY, 1995.

Chan, V.S.S., Robinson, D.I., Turner, J.T., and Heyes, A.L. "A Simplified Doppler Global Velocimeter," Vol. 229, pp. 9-14, ASME FED Laser Anemometry Proceedings of the 1995 ASME/JSME Fluids Engineering and Laser Anemometry Conference and Exhibition, Hilton Head, SC, Aug. 13-18 1995.

Clancy, P.S., Samimy, M., and Erskine, W.R., "Planar Doppler Velocimetry: ThreeComponent Velocimetry in Supersonic Jets," Paper 98-0506, AIAA $36^{\text {th }}$ Aerospace Sciences Meeting and Exhibit, Reno, NV, Jan. 12-15, 1998.

Crafton, J., Messersmith, N.M., and Sullivan, J.P., "Filtered Doppler Velocimetry: Development of a Point System," AIAA Paper 98-0509, AIAA $36^{\text {th }}$ Aerospace Sciences Meeting \& Exhibit, Reno, NV, Jan. 12-15, 1998. 
Elliot, G.S., Samimy, M., and Arnette, S.A., "Details of a Molecular Filter-Based Velocimetry Technique," AIAA Paper 94-0490, AIAA 32 ${ }^{\text {nd }}$ Aerospace Sciences Meeting, Reno, NV, Jan. 10-13, 1994.

Ford, H. D. and Tatam, R. P., "Imaging System Considerations in Doppler Global Velocimetry," Vol. 2546, Proceedings of SPIE - The International Society for Optical Engineering, Optical Techniques in Fluid, Thermal, and Combustion Flow, San Diego, CA, July 10-13, 1995.

Forkey, J.N., Finkelstein, N.D., Lempert, W.R., and Miles, R.B., "Demonstration and Characterization of Filtered Rayleigh Scattering for Planar Velocity Measurements," AIAA Journal Vol. 34, No. 3, pp. 442-448. Mar. 1996.

Gorton, S.A., Meyers, J.F., and Berry, J.D., "Velocity Measurements Near the Empennage of a Small-Scale Helicopter Model," Proceedings of the 1996 American Helicopter Society 52nd Annual Forum, Vol. 1, pp 517-530, Washington, DC, June 4-6, 1996.

Hoffenberg, R., and Sullivan, J.P., " Filtered Particle Scattering: Laser Velocimetry Using An Iodine Filter," ASME-FED, vol. 161, pp. 135-138, June 20-24, 1993.

Irani, E., "Application and Evaluation of the Doppler Global Velocimetry Method on an Axi-Symmetric Jet," PhD Dissertation, Wichita State University, May, 1995.

Irani, E. and Miller, L.S., "Evaluation of a Basic Doppler Global Velocimetry System," Paper 951427, SAE Aerospace Atlantic Conference, Dayton, OH, May 23-25, 1995.

James, K.M., "Determination of the Accuracy of a Two-Component Point Doppler Velocimetry System," MS thesis, Department of Mechanical and Aerospace Engineering, College of Engineering, West Virginia University, June 1997.

Komine, H., United States Patent, Patent No. 4,919,536, pp. 1-16, April 24, 1990.

Komine, H., and Brosnan, S.J., "Instantaneous, Three-Component, Doppler Global Velocimetry," Laser Anemometry - Vol. 1, pp. 273-277, ASME 1991.

Komine, H., Brosnan, S.J., Litton, A.B., and Stappaerts, E.A., "Real-Time Doppler Global Velocimetry," AIAA Paper 91-0337, 29th Aerospace Sciences Meeting, Reno, NV, January 7-10, 1991.

Kuhlman, J. M., “Turbulence Measurements in Annular Jets Using Laser Velocimetry (LV)," ASME Fluids Engineering Division Summer Meeting, Vol.191, pp. 77-82, Lake Tahoe, NV, June 19-23, 1994. 
Kuhlman, J., Naylor, S., James, K., and Ramanath, S., "Accuracy Study of a 2Component Point Doppler Velocimeter (PDV)," AIAA Paper 97-1916, $35^{\text {th }}$ Aerospace Sciences Meeting and Exhibit, Reno, NV, Jan. 6-10, 1997.

Leporcq, B., Le Roy, J.F., Pinchemel, B., and Dufour, C. "Interest of a Cw Dye Laser in Doppler Global Velocimetry," Proceedings of the ASME Fluids Engineering Division Summer Meeting, Vol. 239 No. 4, pp. 115-122, San Diego, CA, Jul 7-11 1996.

McKenzie, R.L., "Measurement Capabilities of Planar Doppler Velocimetry Using Pulsed Lasers," AIAA Paper 95-0297, 33rd Aerospace Sciences Meeting and Exhibit, Reno, NV, Jan. 9-12, 1995.

McKenzie, R.L., "Measurements Capabilities of Planar Doppler Velocimetry using Pulsed Lasers," Applied Optics, Vol. 35, No.6, pp. 948-964. February 20, 1996.

McKenzie, R. L., "Planar Doppler Velocimetry Performance in Low-Speed Flows," AIAA Paper 97-0498, AIAA $35^{\text {th }}$ Aerospace Sciences Meeting and Exhibit, Reno, NV, Jan. 6-10, 1997.

McKenzie, R. L., "Planar Doppler Velocimetry for Large-Scale Wind Tunnel Applications," Paper No. 9, AGARD Fluid Dynamics Panel $81^{\text {st }}$ Meeting and Symposium on Advanced Aerodynamic Measurement Technology, Seattle, WA, Sept. 22-25, 1997.

Menders, J., Benson, K., Bloom, S.H., Liu, C.S., and Korevaar, E., "Ultranarrow Line Filtering Using a Cs Faraday Filter at 852 nm," Optics Letters, Vol. 16, No. 11, pp. 846-848, June 1, 1991.

Meyers, J.F. and Komine, H., "Doppler Global Velocimetry: A New Way to Look at Velocity," ASME Fourth International Conference on Laser Anemometry, Cleveland, OH, Aug. 3-9, 1991.

Meyers, J.F., Lee, J.W., and Cavone, A.A., " Signal Processing Schemes for Doppler Global Velocimetry," IEEE 14th International Congress on Instrumentation in Aero Simulation Facilities (ICIASF), Rockville, MD, October 27-31,1991.

Meyers, J.F., "Doppler Global Velocimetry The Next Generation?," AIAA 27th Aerospace Ground Testing Conference, Nashville TN, July 6-8, 1992.

Meyers, J.F., "Development of Doppler Global Velocimetry For Wind Tunnel Testing," 18th AIAA Aerospace Ground Testing Conference, Colorado Springs, CO, June 20-23, 1994. 
Meyers, J.F., "Evolution of Doppler Global Velocimetry Data Processing," Eighth International Symposium on Applications of Laser Techniques to Fluid Mechanics, Lisbon, Portugal, pp. 1-22, July 8-11, 1996.

Meyers, J.F., Personal Communication. 1997.

Miles, R.B., Forkey, J.N., and Lempert, W.R., "Filtered Rayleigh Scattering Measurements in Supersonic/Hypersonic Facilities," AIAA 17th Aerospace Ground Testing Conference, Nashville, TN, July 6-8, 1992.

Miles, R.B., and Lempert, W.R., "Three-Dimensional Diagnostics in Air and Water by Molecular Tagging and Molecular Scattering," 27th AIAA Fluid Dynamics Conference, New Orleans, LA, June 17-20, 1996.

Morrison, G.L., Gaharan, C.A., and DeOtte, R.E., Jr., ' Doppler Global Velocimetry, Problems and Pitfalls," Laser Anemometry 1994 Advances and Applications, ASME Publication FED-Vol. 191, June 1994.

Naylor, S., Kuhlman, J., “Accuracy Studies of a Two-Component Doppler Global Velocimeter (DGV),"AIAA Paper 98-0508, 36 ${ }^{\text {th }}$ Aerospace Sciences Meeting \& Exhibit, Reno, NV, Jan. 12-15, 1998.

Press, W. H., Teukolsky, S. A., Vetterling, W. T., and Flannery, B. P., Numerical Recipes in FORTRAN, $2^{\text {nd }}$ Edition, Cambridge University Press, 1992.

Rae, W.H. and Pope, A., Low Speed Wind Tunnel Testing, 2nd Ed., John Wiley and Sons, Inc., pp. 100-125, 1984.

Reeder, M., Samimy, M., and Elliot, G.S., "An Investigation of the Effect of Tabs on Jets Using Advanced Diagnostics," AIAA Paper 95-0672, $33^{\text {rd }}$ Aerospace Sciences Meeting and Exhibit, Reno, NV, Jan. 9-12, 1995.

Reeder, M.F., "Jet Entrainment Measurements Via Doppler Global Velocimetry," Proceedings of the ASME Fluids Engineering Division Summer Meeting, Vol. 239 No. 4, pp. 129-134, San Diego, CA, Jul 7-11 1996.

Reinath, Michael S., "Doppler Global Velocimeter Development for the Large Wind Tunnels at Ames Research Center," NASA Technical Memorandum 112210, Sept. 1997.

Roehle, I. and Schodl, R., "Evaluation of the Accuracy of the Doppler Global Technique," Optical Methods and Data Processing in Heat and Fluid Flow, London, UK, pp.155-161, April 14-15, 1994. 
Roehle, I., "Three-Dimensional Doppler Global Velocimetry in the flow of a fuel spray nozzle and in the wake region of a car," Flow Measurement and Instrumentation, Vol. 7, No. 3-4, Sept-Dec, 1996.

Seasholtz, R.G., "Instantaneous 2D Velocity and Temperature Measurements in High Speed Flows Based on Spectrally Resolved Rayleigh Scattering," AIAA Paper 950300, $33^{\text {rd }}$ Aerospace Sciences Meeting and Exhibit, Reno, NV, January 9-12, 1995.

Smith, M.W., and Northam, G.B., "Application of Absorption Filter-Planar Doppler Velocimetry to Sonic and Supersonic Jets," AIAA Paper 95-0299, 33 $3^{\text {rd }}$ Aerospace Sciences Meeting and Exhibit, Reno, NV, pp.1-14, January 9-12, 1995.

Smith, M.W., Northam, G.B., and Drummond, J.P., "Application of Absorption Filter Planar Doppler Velocimetry to Sonic and Supersonic Jets," AIAA Journal, Vol. 34, No. 3, pp. 434-441, March 1996.

Smith, M. W., "Application of a Planar Doppler Velocimetry System to a High Reynolds Number Compressible Jet," AIAA Paper 98-0428, $36^{\text {th }}$ Aerospace Sciences Meeting and Exhibit, Reno, NV, Jan. 12-15, 1998.

Smith, M., "The Reduction of Laser Speckle Noise in Planar Doppler Velocimetry," AIAA Paper 98-2607, 20 ${ }^{\text {th }}$ Advanced Measurement and Ground Testing Technology Conference, Albuquerque, NM, June 15-18, 1998.

Thorpe, S.J., Ainsworth, R.W., and Manners, R.J., "The Development of a Doppler Global Velocimeter and its Application to a Free Jet Flow," FED-Vol 229, Laser Anemometry, ASME, 1995.

Thorpe, S.J., Ainsworth, R.W., and Manners, R.J., "Time Averaged Free Jet Measurements Using Doppler Global Velocimetry,” FED-Vol 239, Fluids Engineering Division Conference, Vol 4, ASME, 1996. 


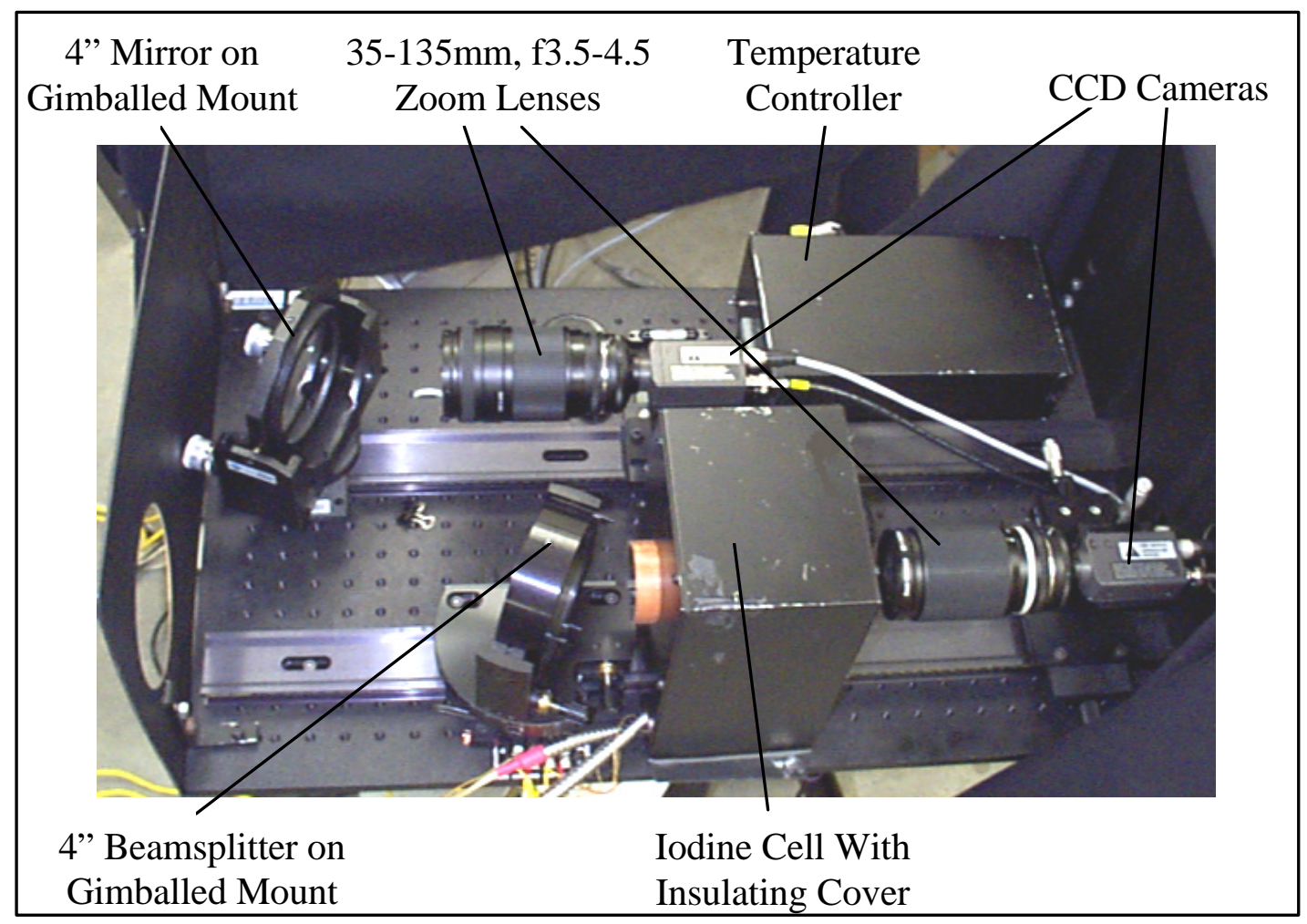

Figure 3.1. Top view of a DGV velocity measuring component

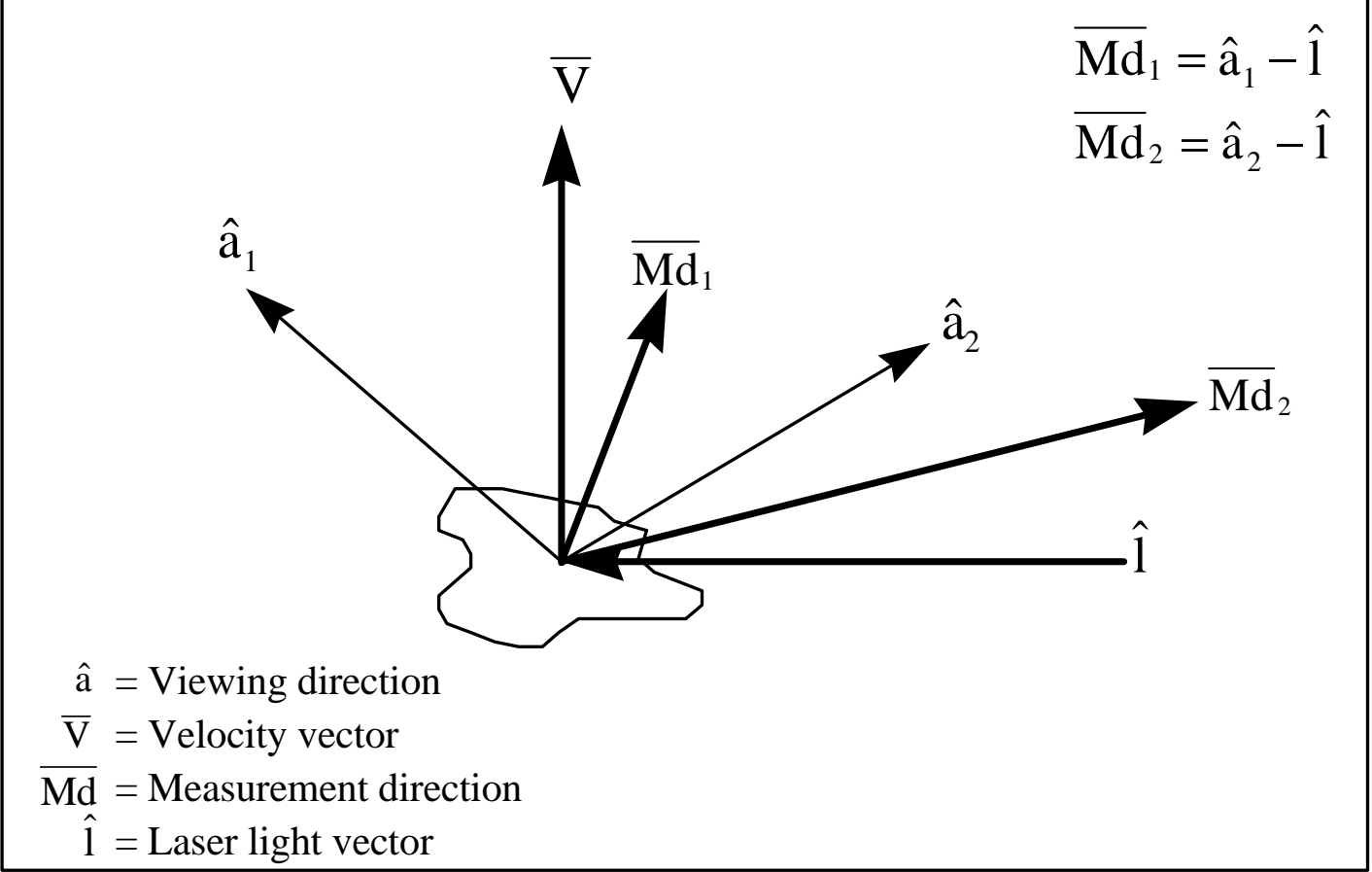

Figure 3.2. Vector geometry for a 2-compontent DGV system 


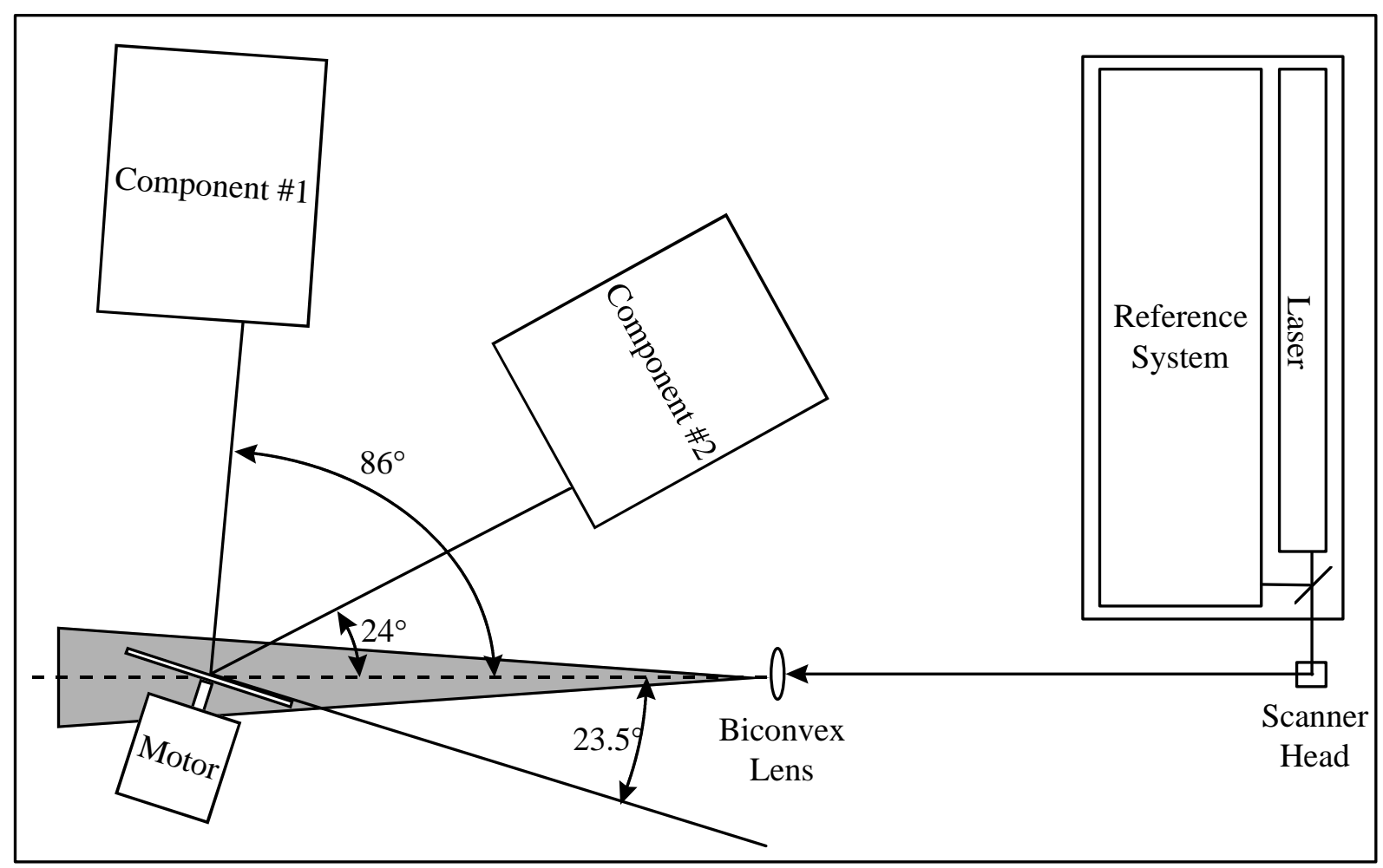

Figure 3.3. Top view schematic of system setup for rotating wheel experiment

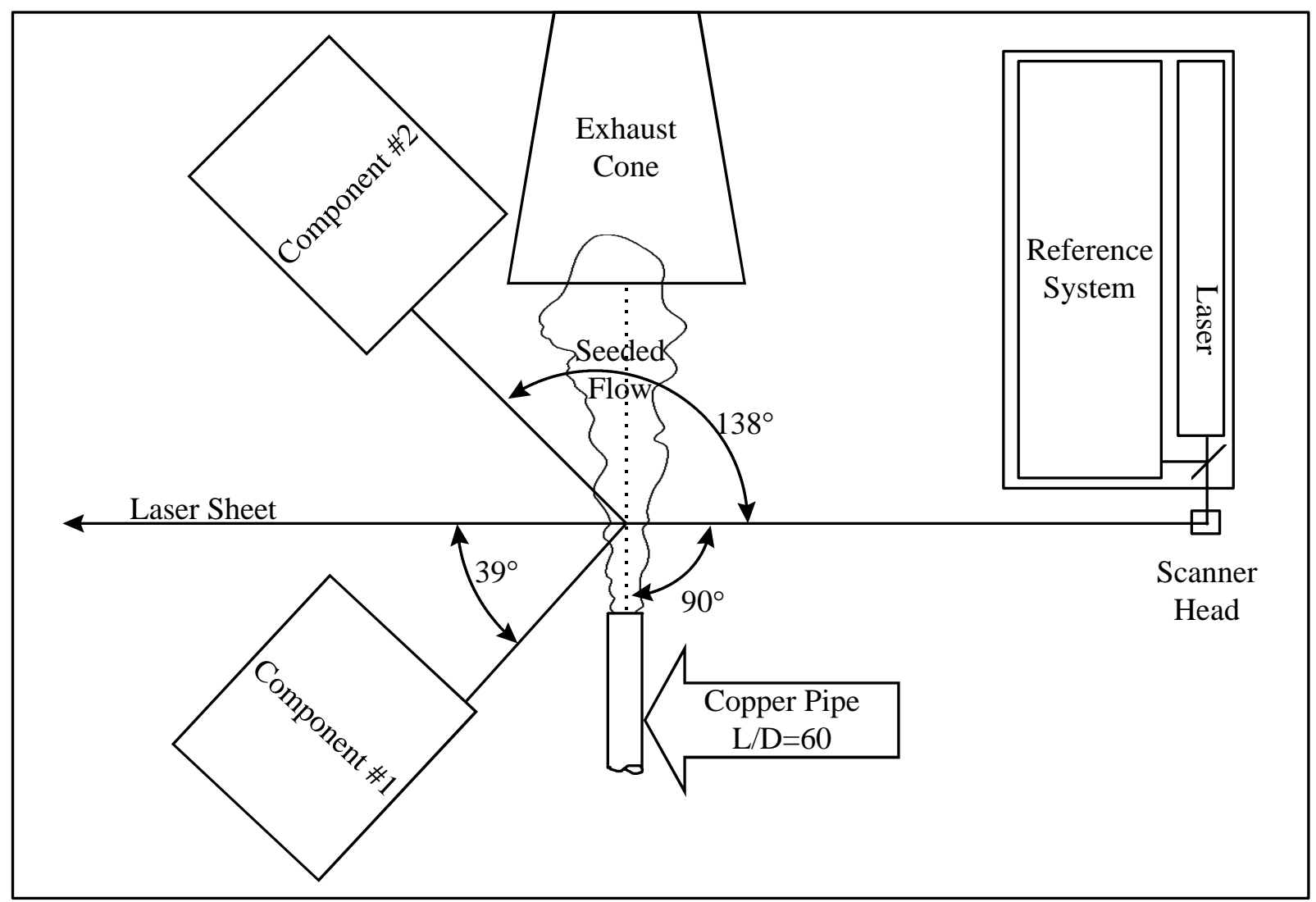

Figure 3.4. Drawing of experimental setup for measurement of turbulent pipe flow 


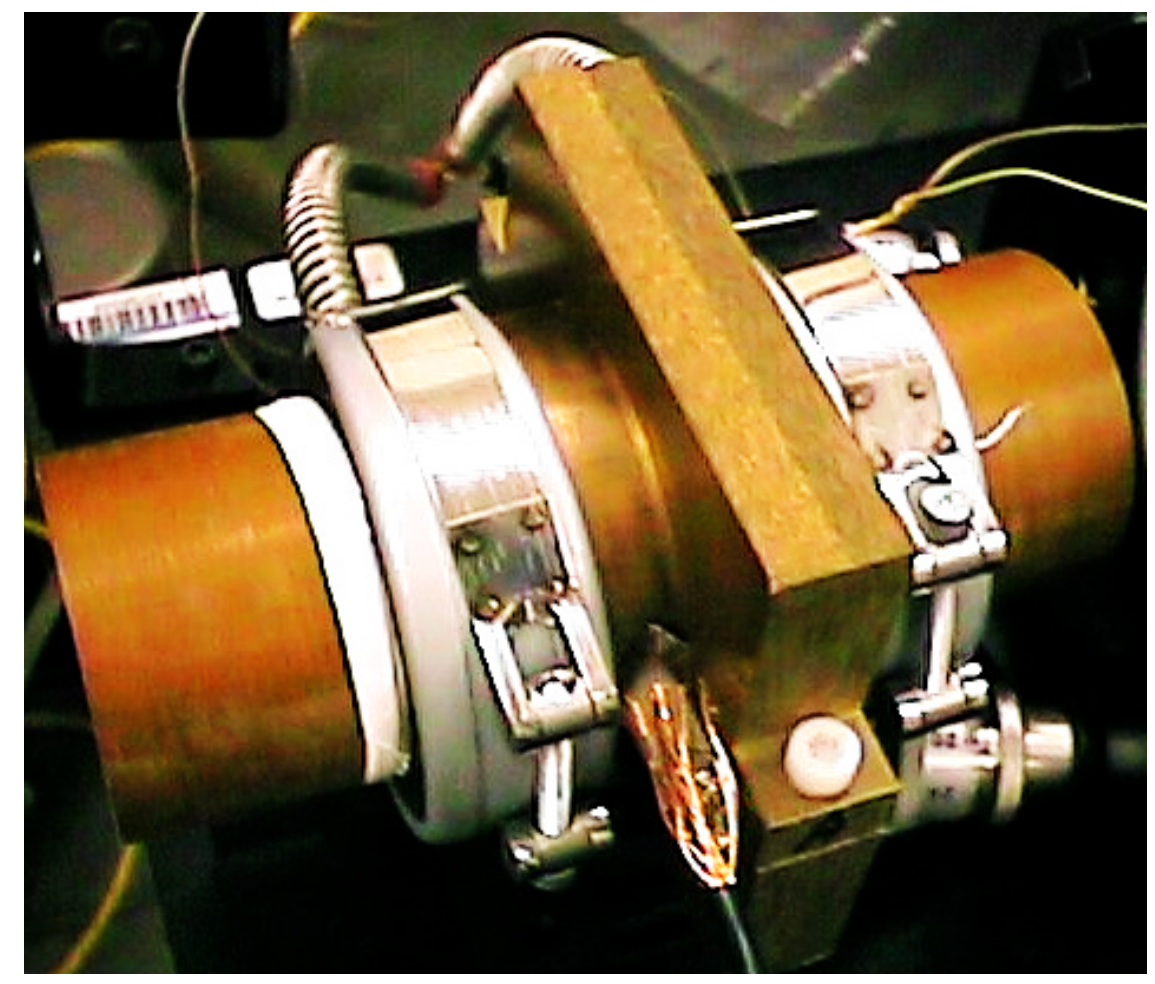

Figure 3.5. Photo of Iodine cell with copper jacket and thermal ground visible

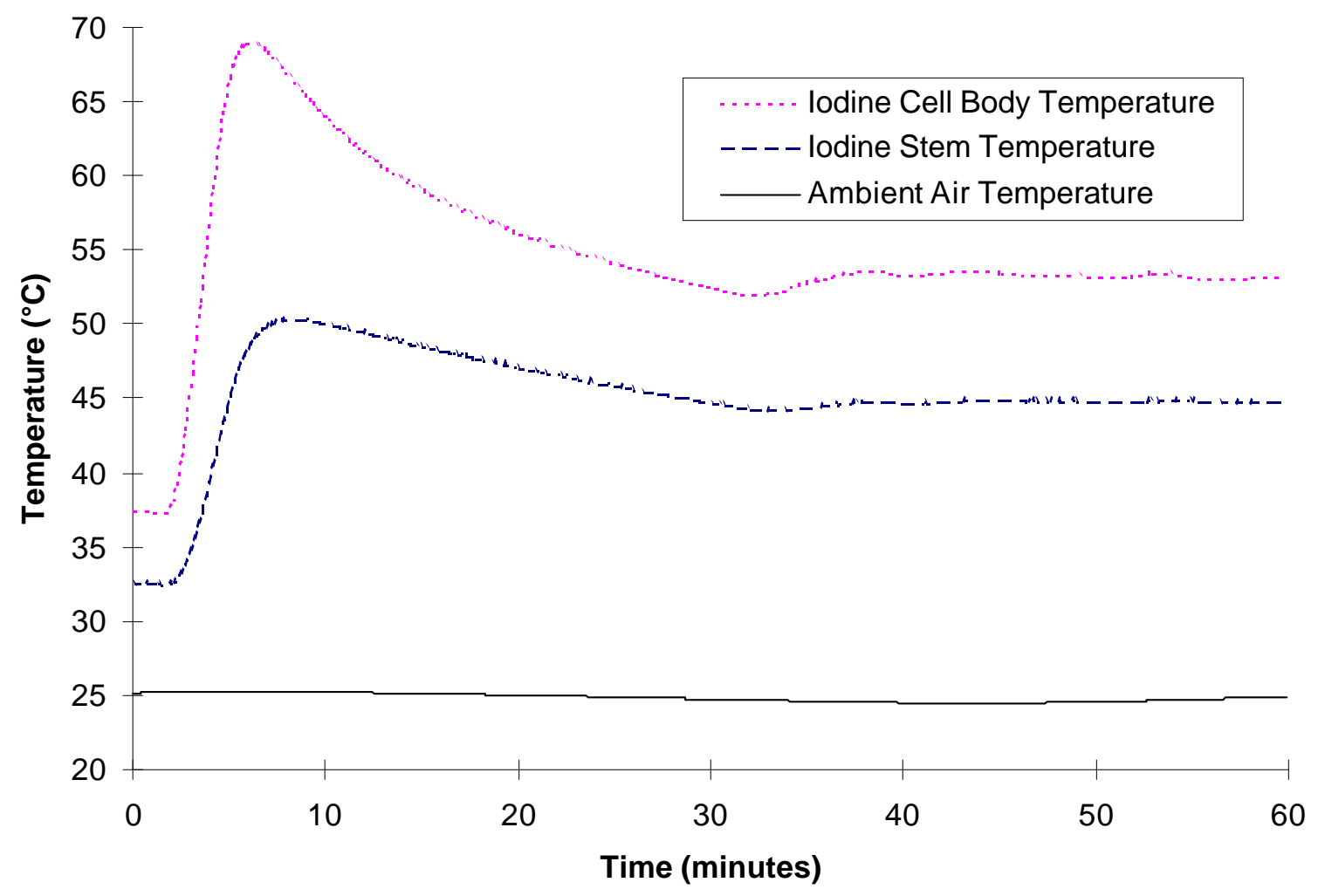

Figure 3.6. Response of temperature controller on $\mathrm{I}_{2}$ cell stem and body temperatures Controller was activated at Time $=2$ minutes 


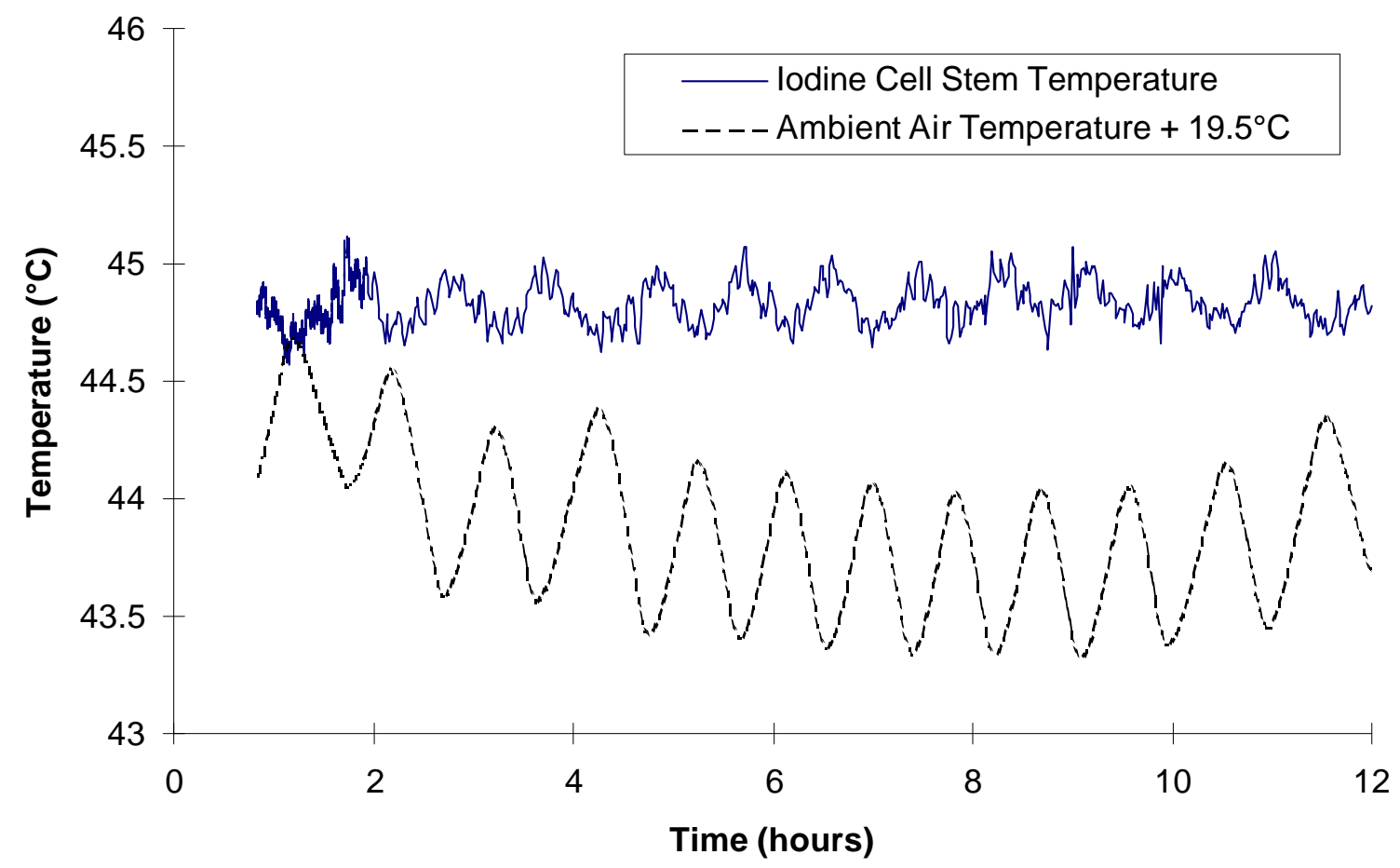

Figure 3.7. Temperature variation of an $\mathrm{I}_{2}$ cell stem showing correlation with ambient temperature

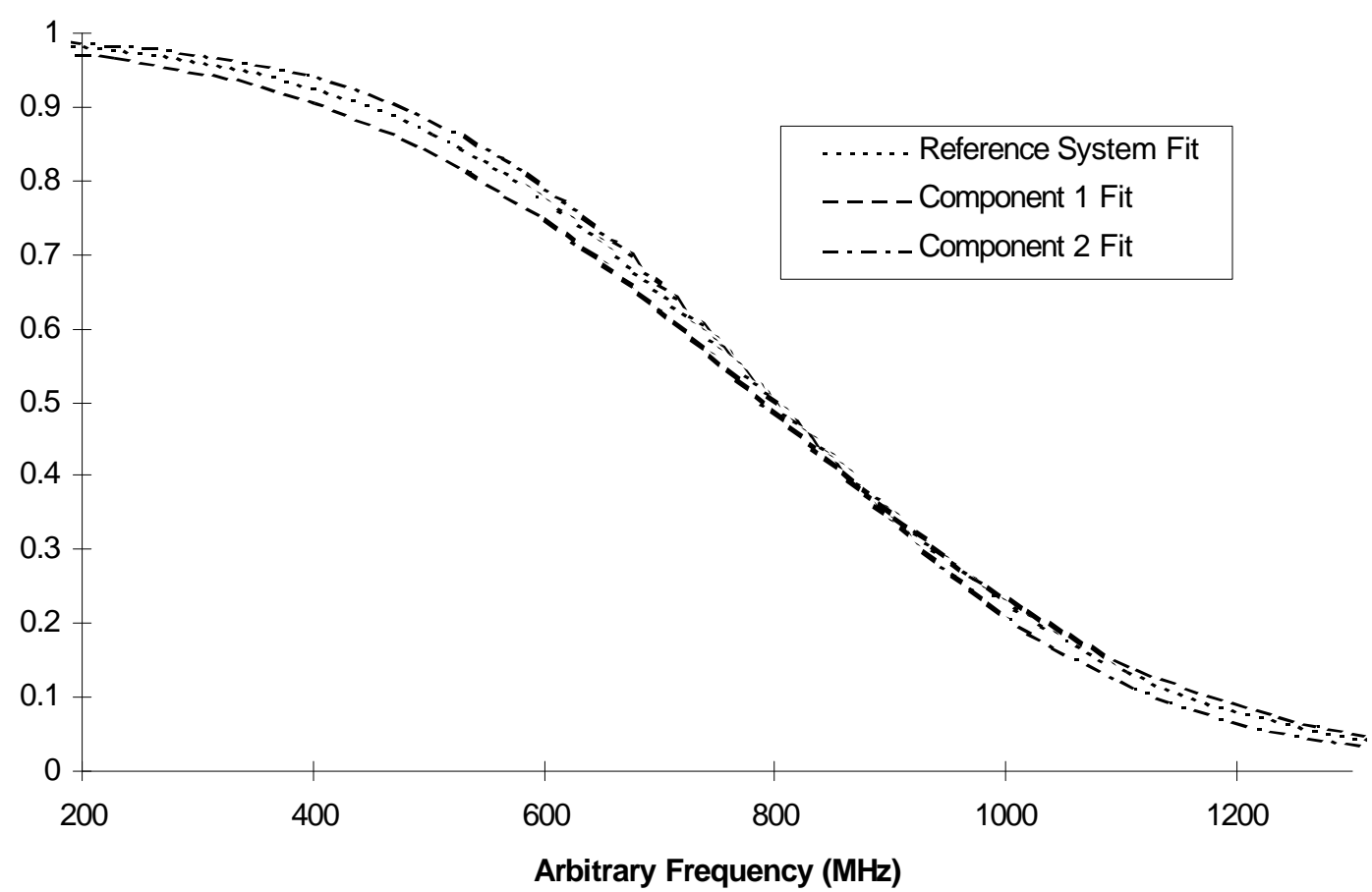

Figure 3.8. Calibration curve fits from all three I2 cells showing slope inconsistencies 


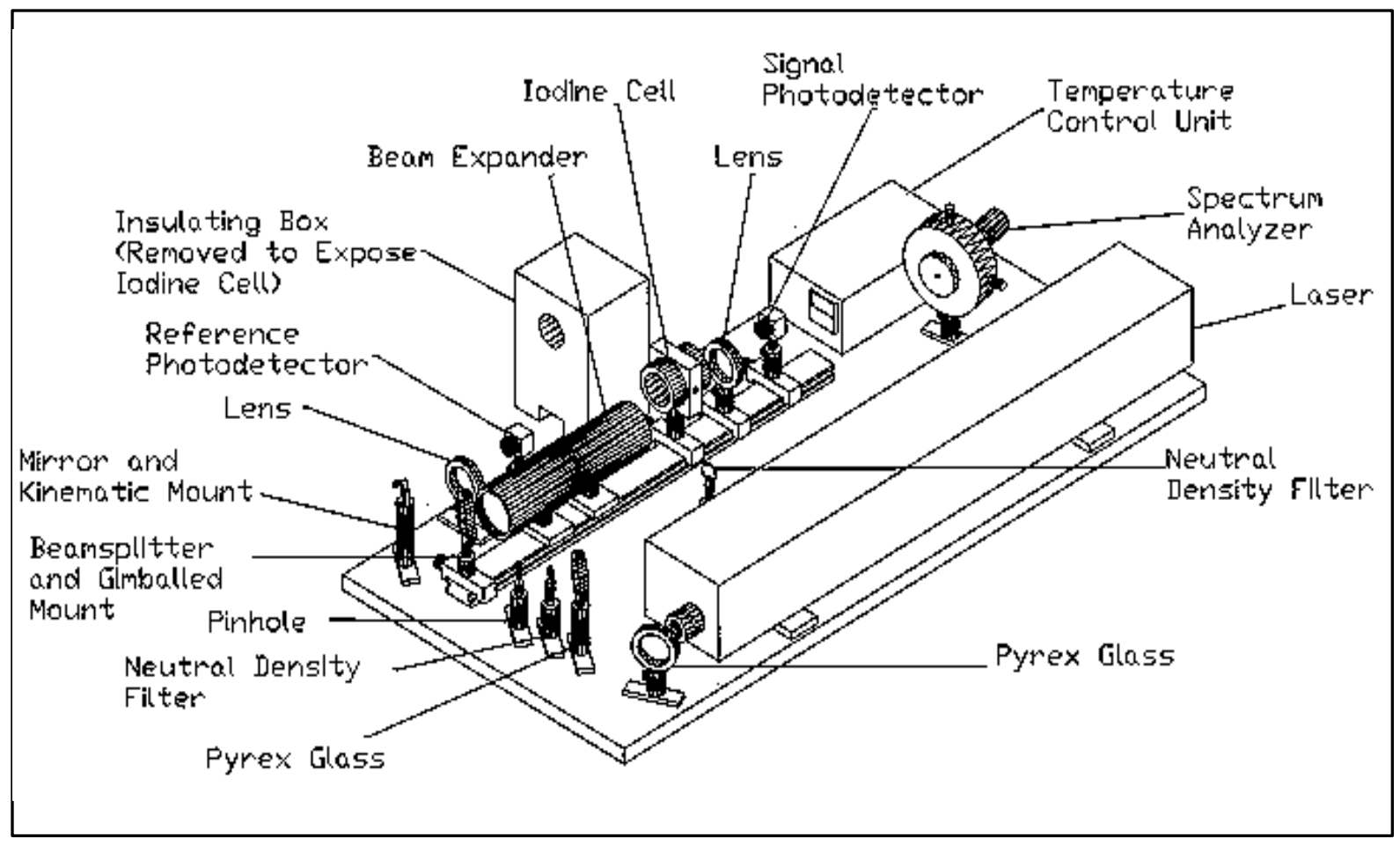

Figure 3.9. Diagram of reference system components

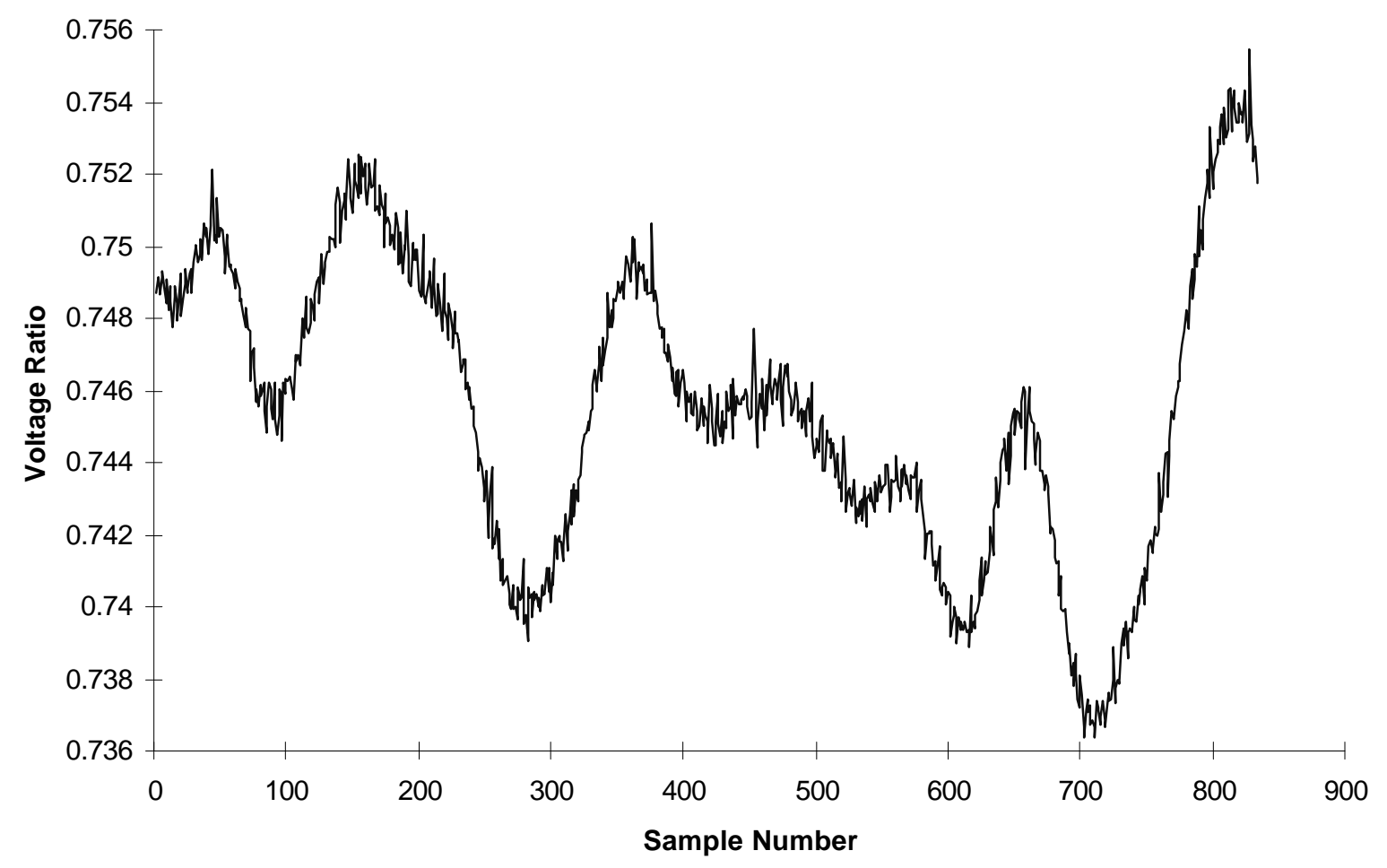

Figure 3.10. Ratio of the voltage outputs of the reference system photodiodes over the time span that an image is acquired $(1 / 60 \mathrm{sec} .=833$ samples $\div 50 \mathrm{kHz})$ 


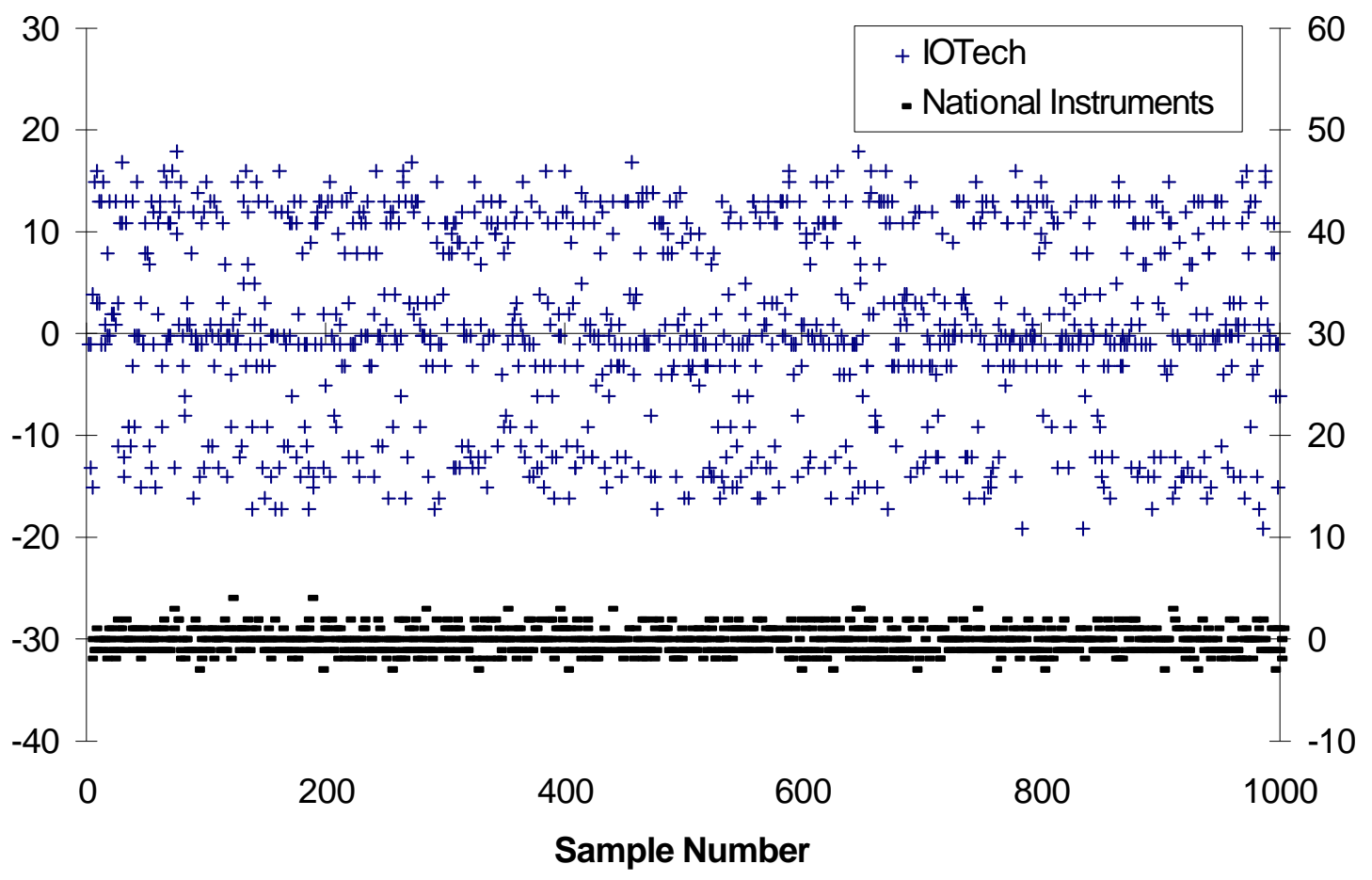

Figure 3.11. Comparison of noise levels from the 16-bit A/D boards when inputs being read are grounded

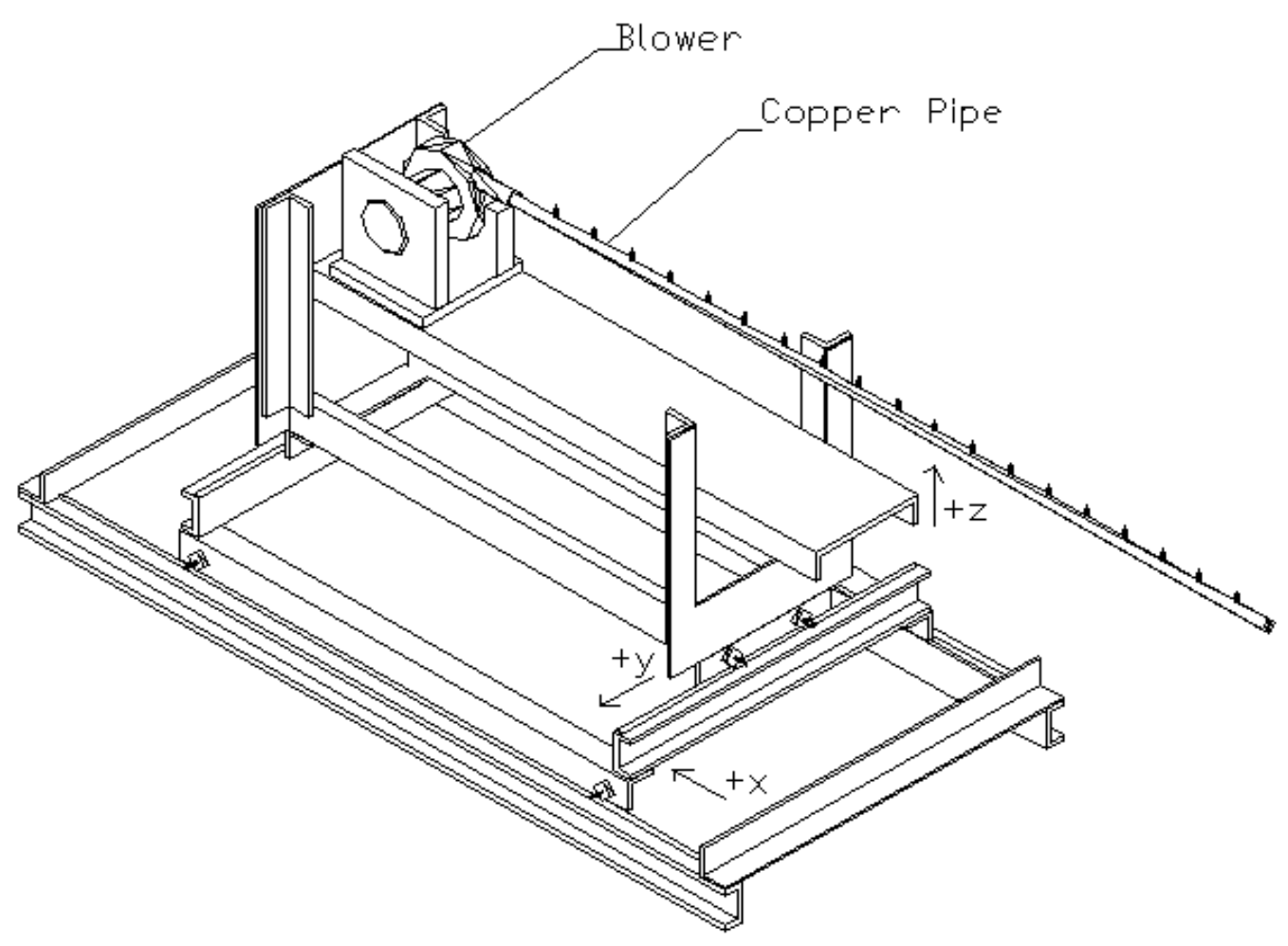

Figure 3.12. Drawing of computer controlled traverse with pipe flow apparatus installed 


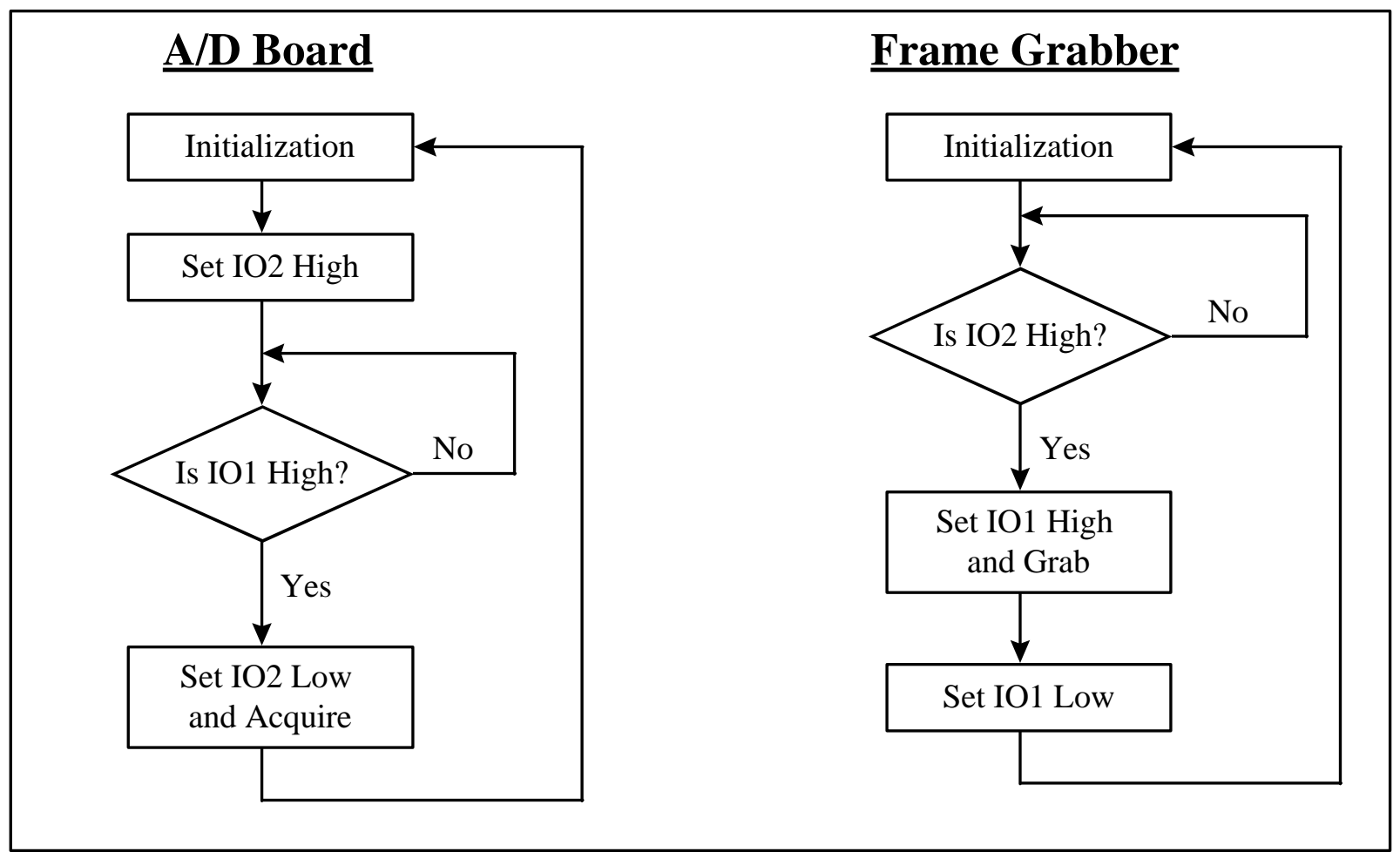

Figure 4.1. Logic to synchronize the frame grabber with the A/D board residing in a separate computer

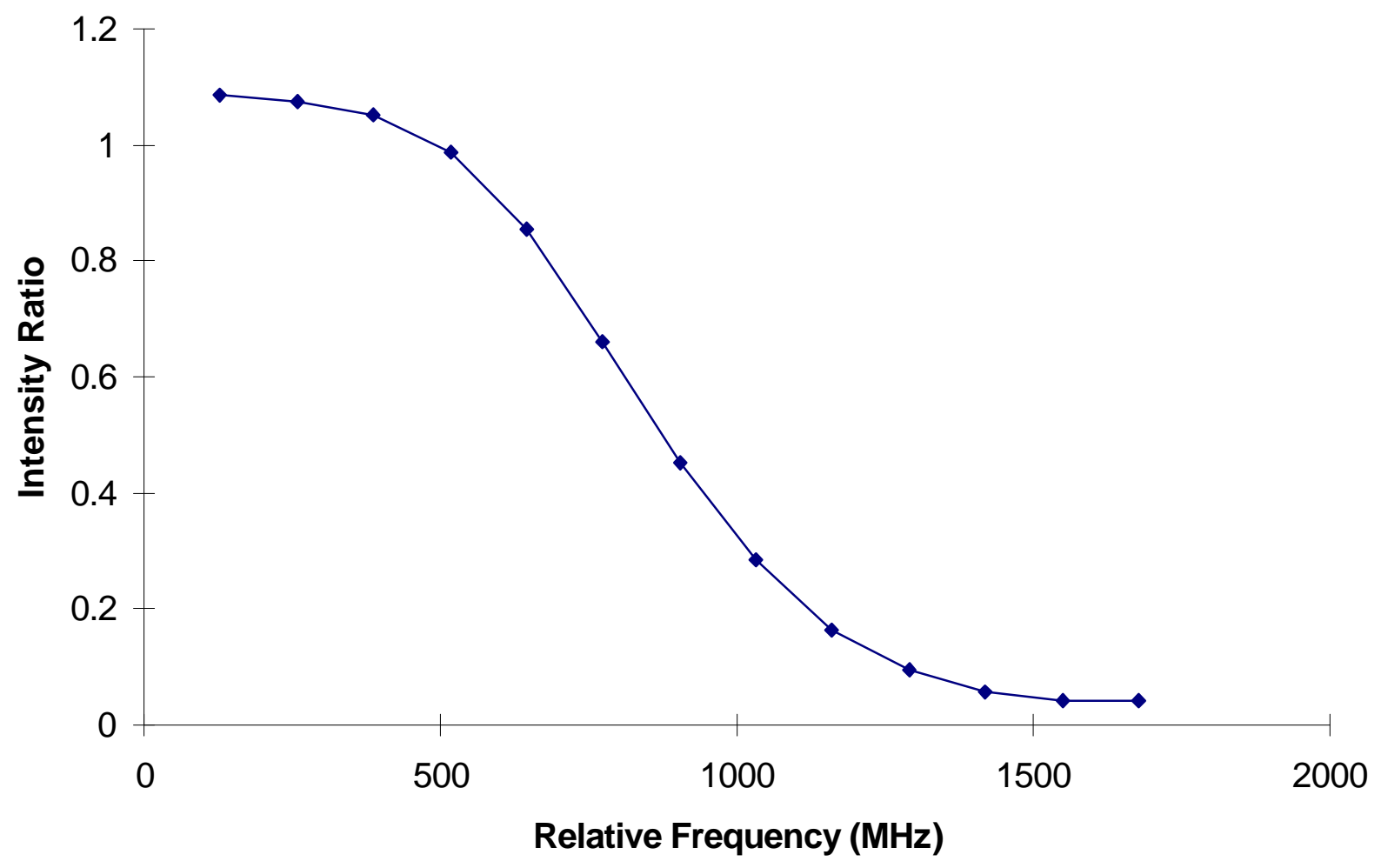

Figure 4.2. Single calibration curve from reference photodetector system showing mode spacing 


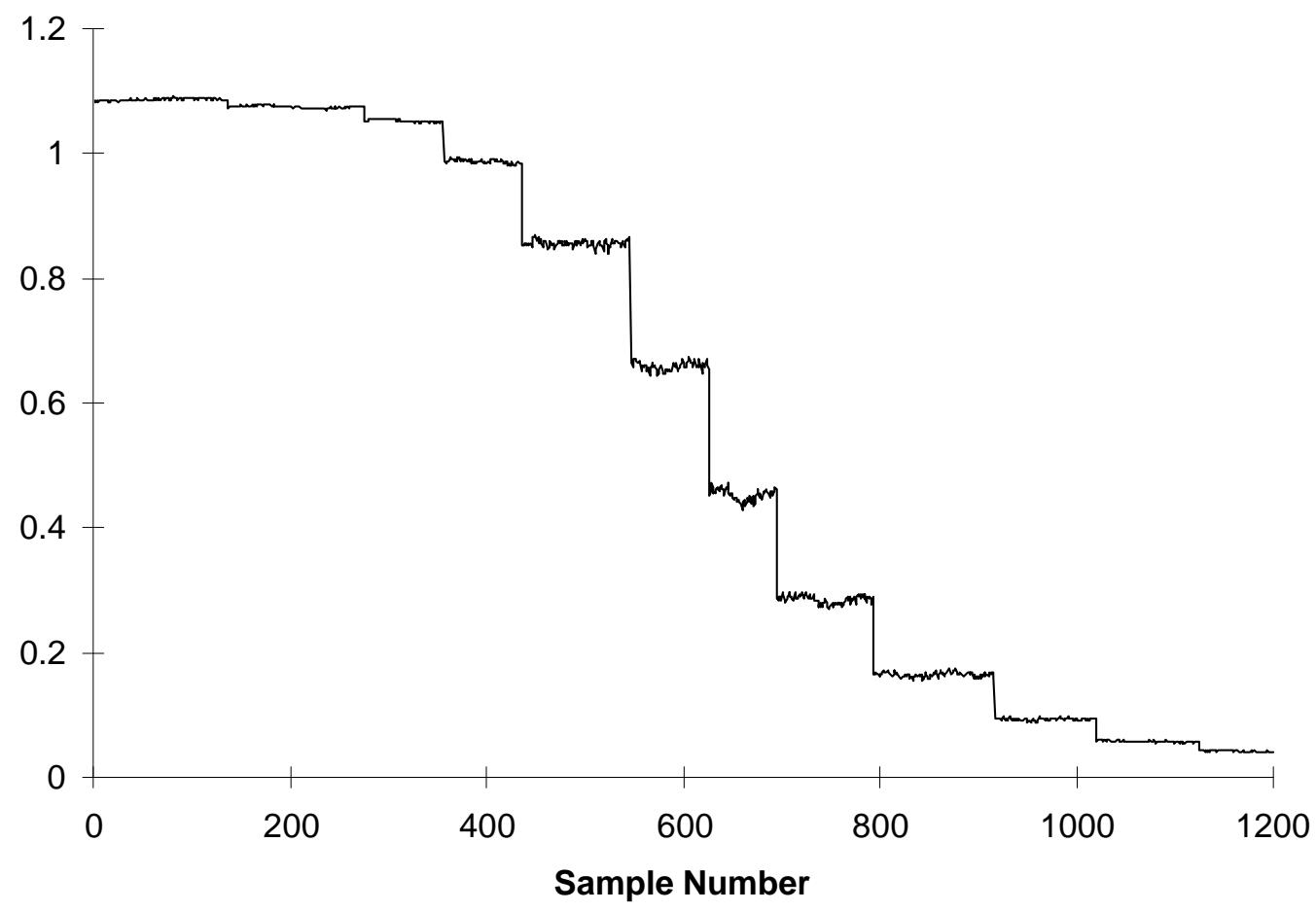

Figure 4.3. Photodetector voltage ratio showing same-mode frequency variations 

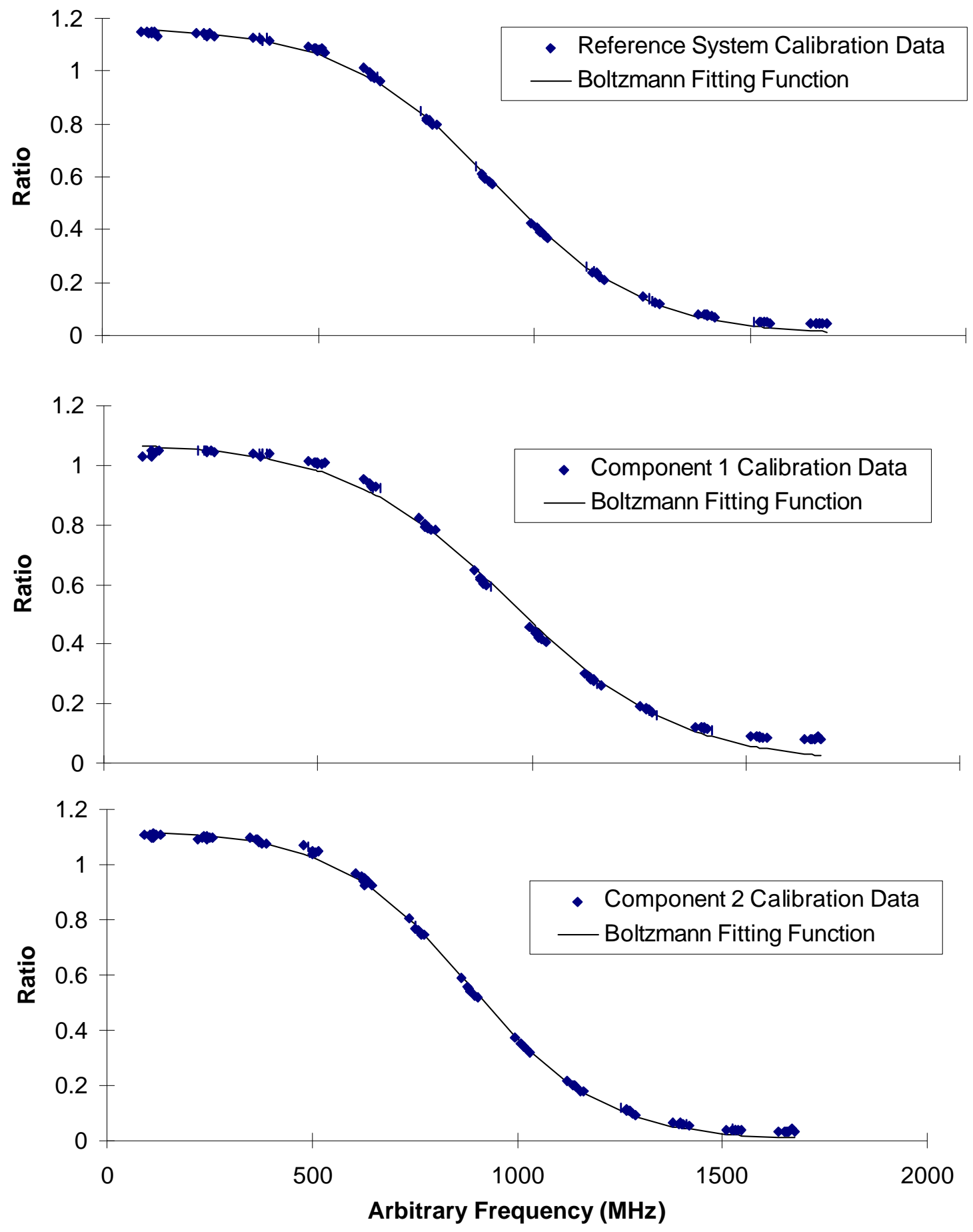

Figure 4.4. Typical calibration data and curve fits for all three iodine cells 


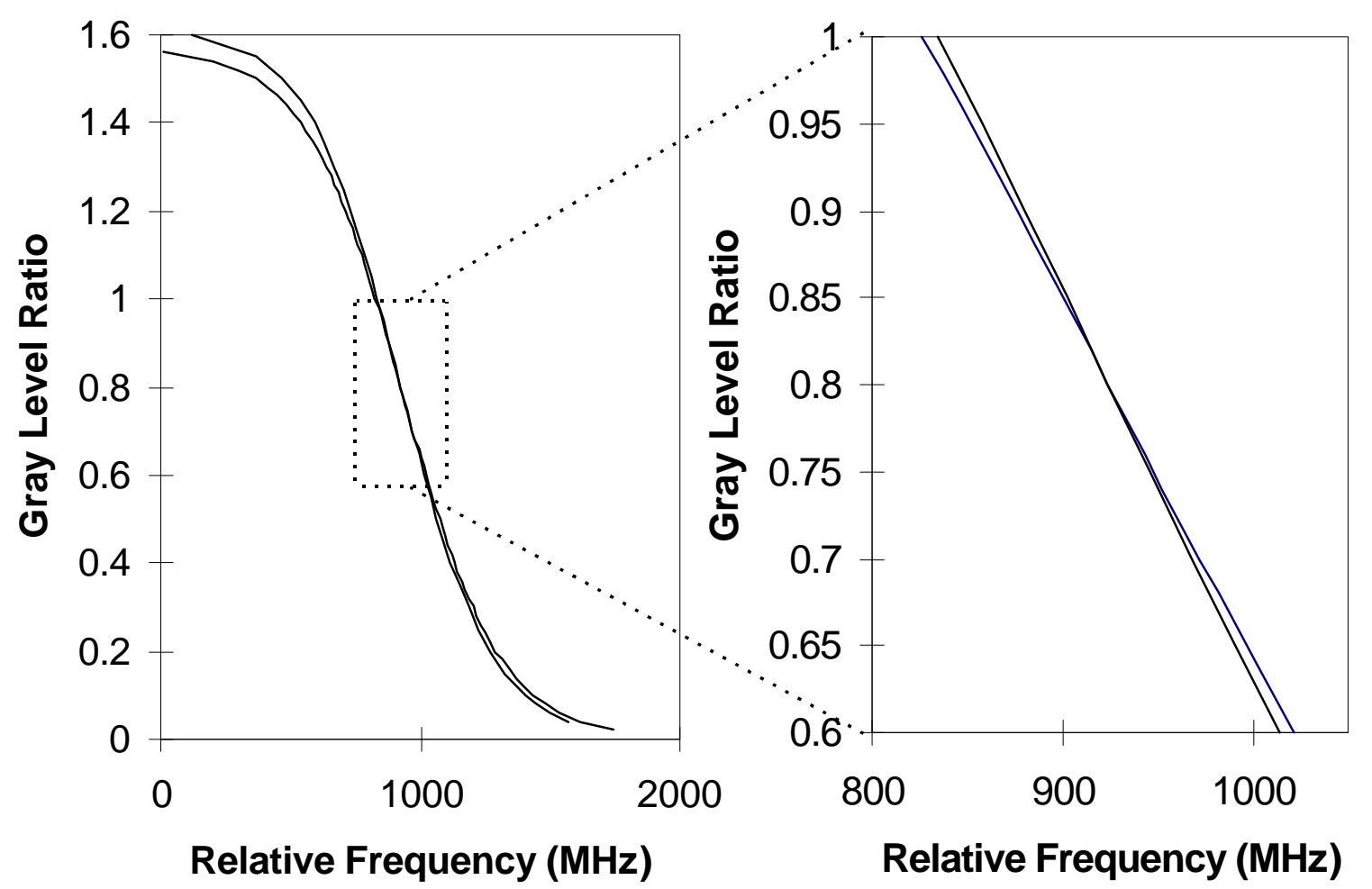

Figure 4.5. Plot of the curve fits for 2 calibrations for cell \#1 with an enlarged view showing variation in slope

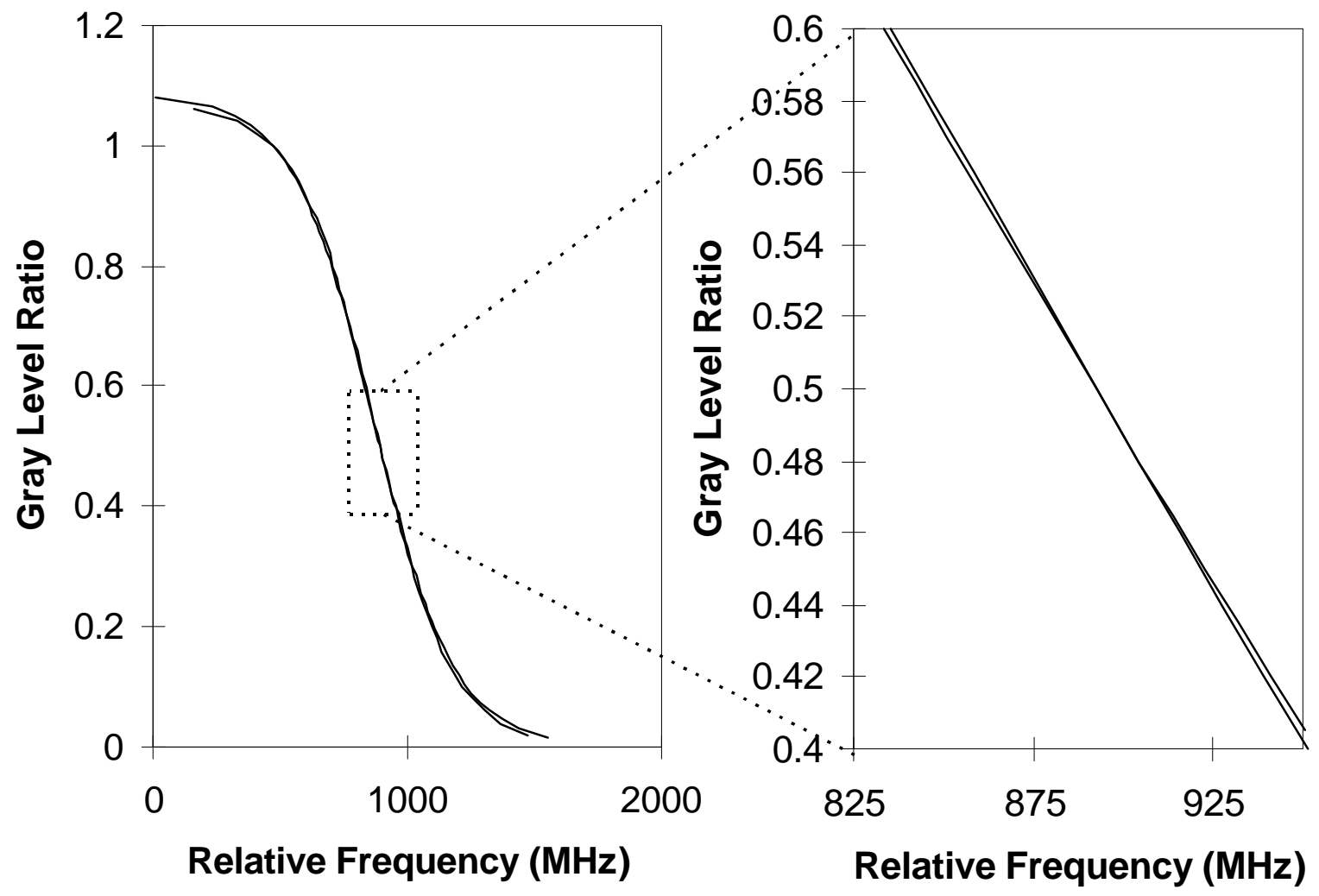

Figure 4.6. Plot of the curve fits for 2 calibrations for cell \#2 with an enlarged view showing variation in slope 

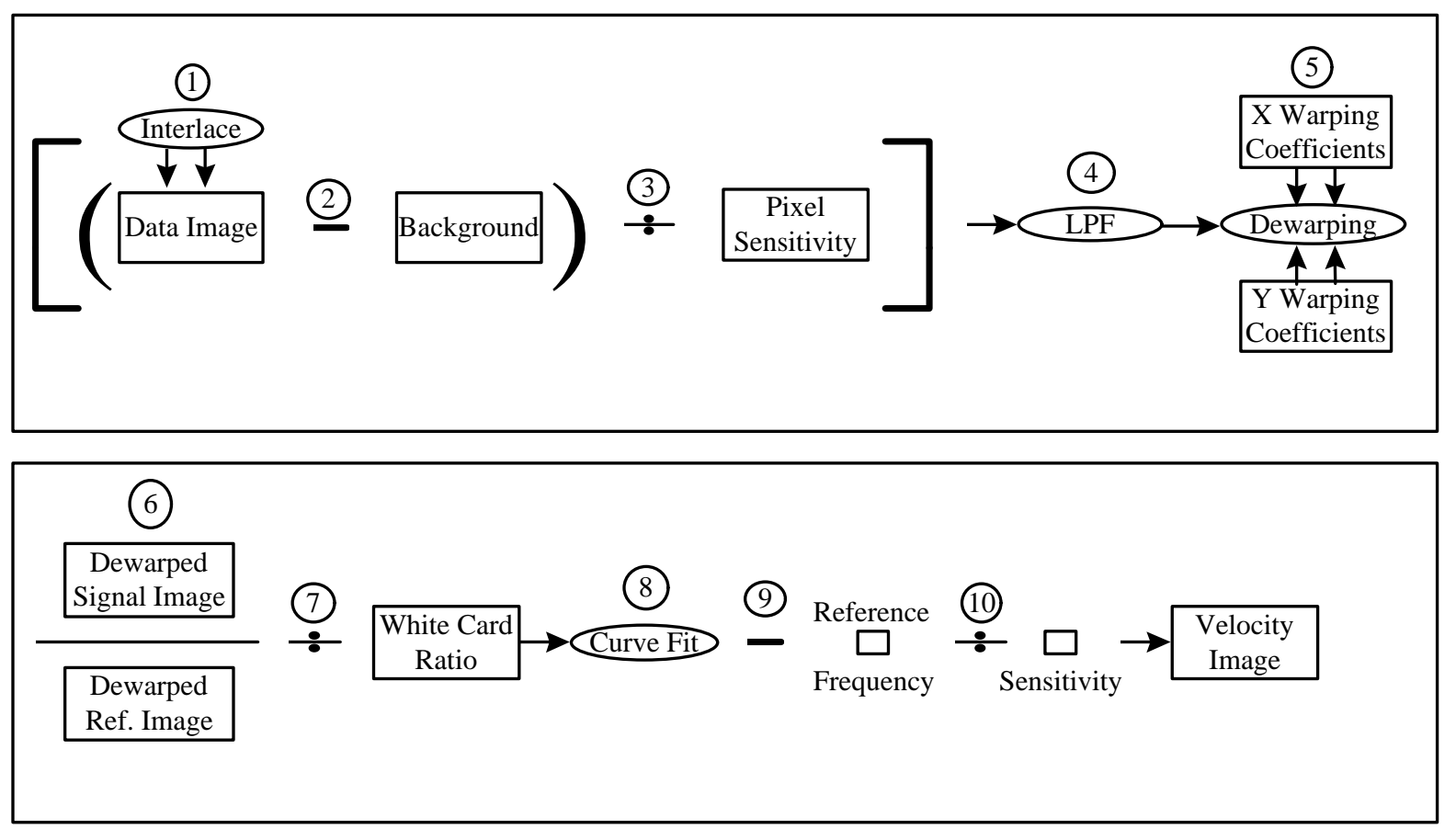

Figure 4.7. Block diagram of image processing steps. Steps 1-5 are performed on each image, while in steps 6-10, signal and reference images are combined to generate a velocity image.

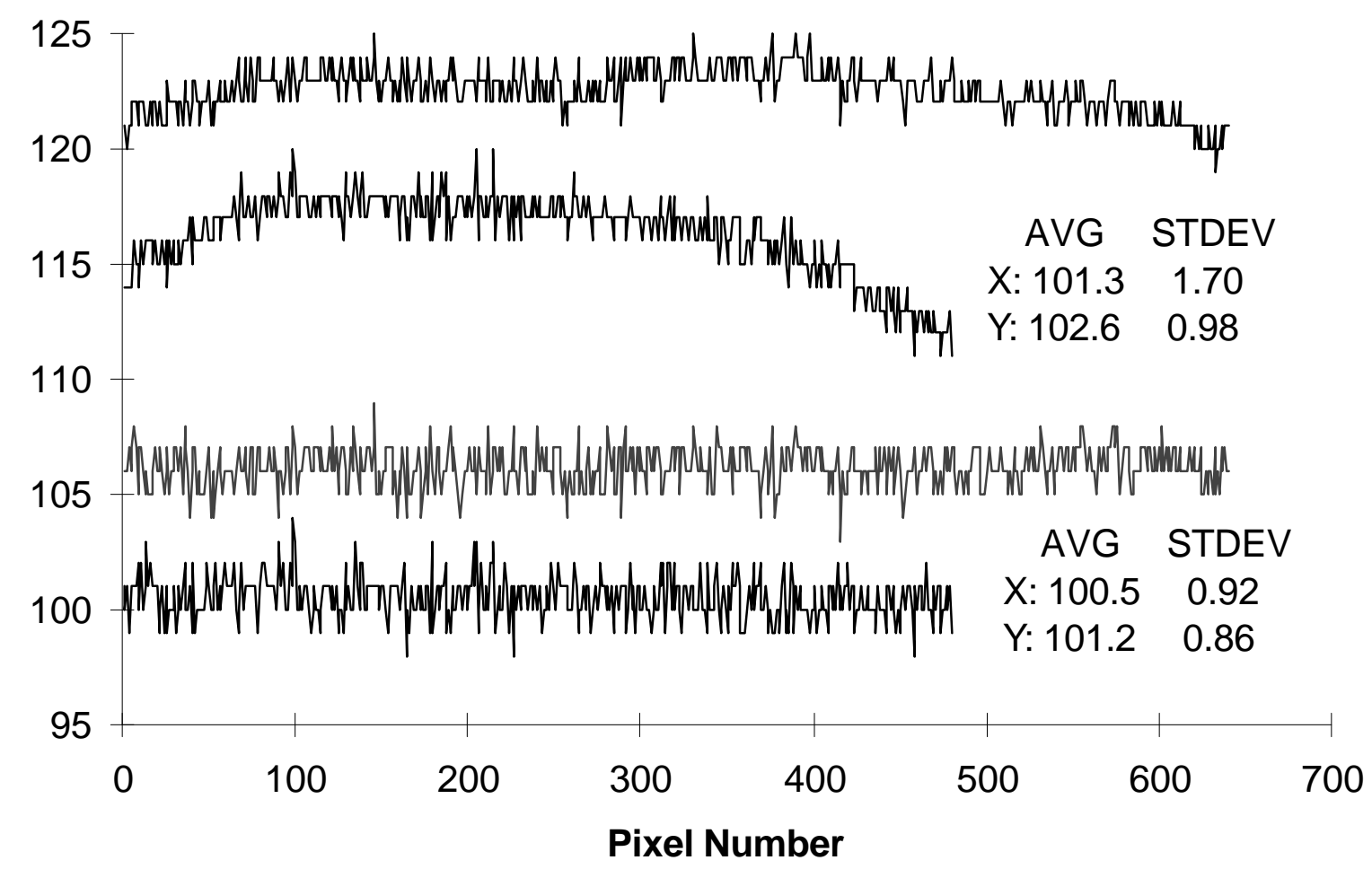

Figure 4.8. Horizontal and vertical cuts through a flatfield image before (top) and after (bottom) applying a pixel sensitivity correction. 


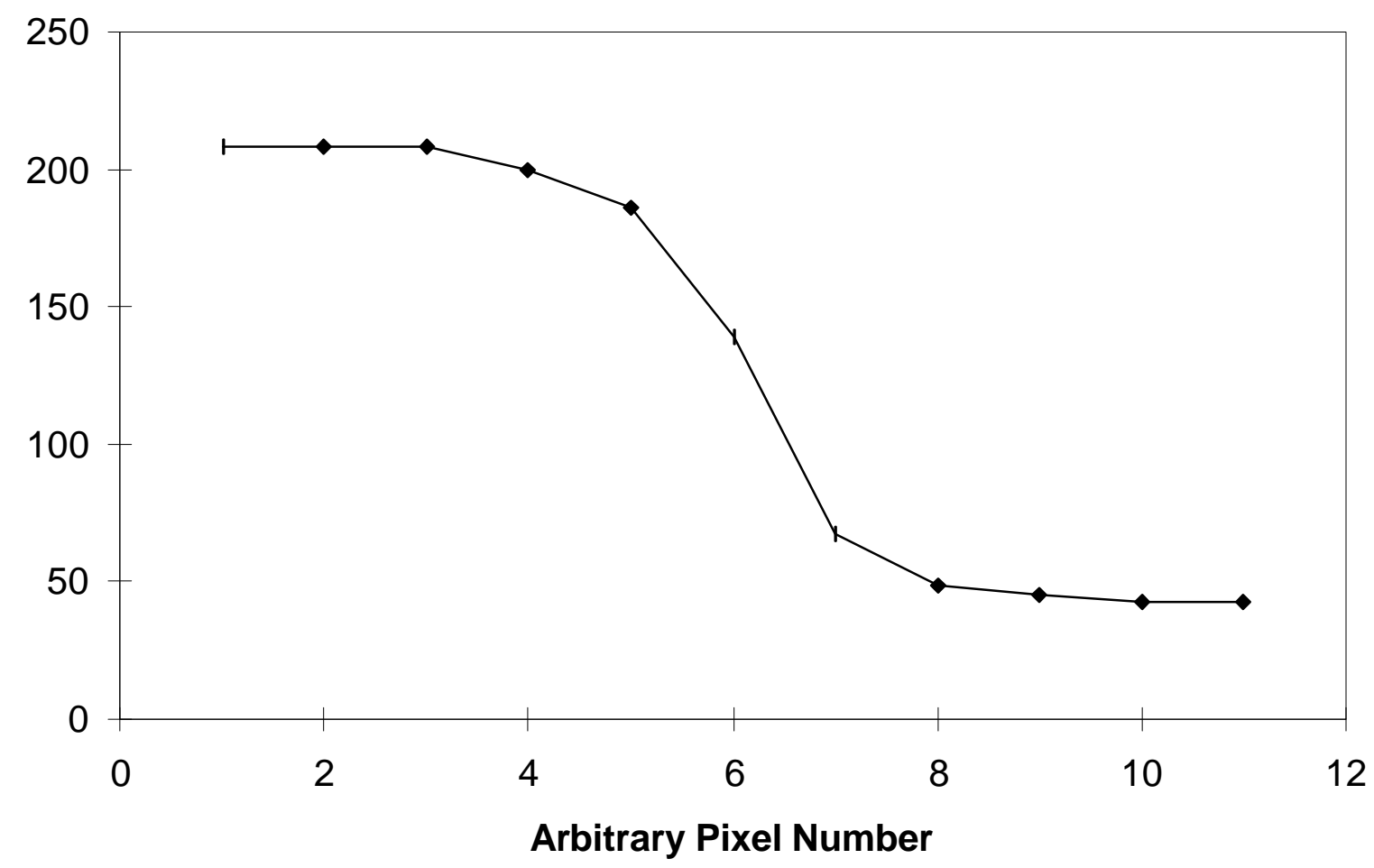

Figure 4.9. Visualization of camera/lens MTF -- Lens f-number approximately 4.5

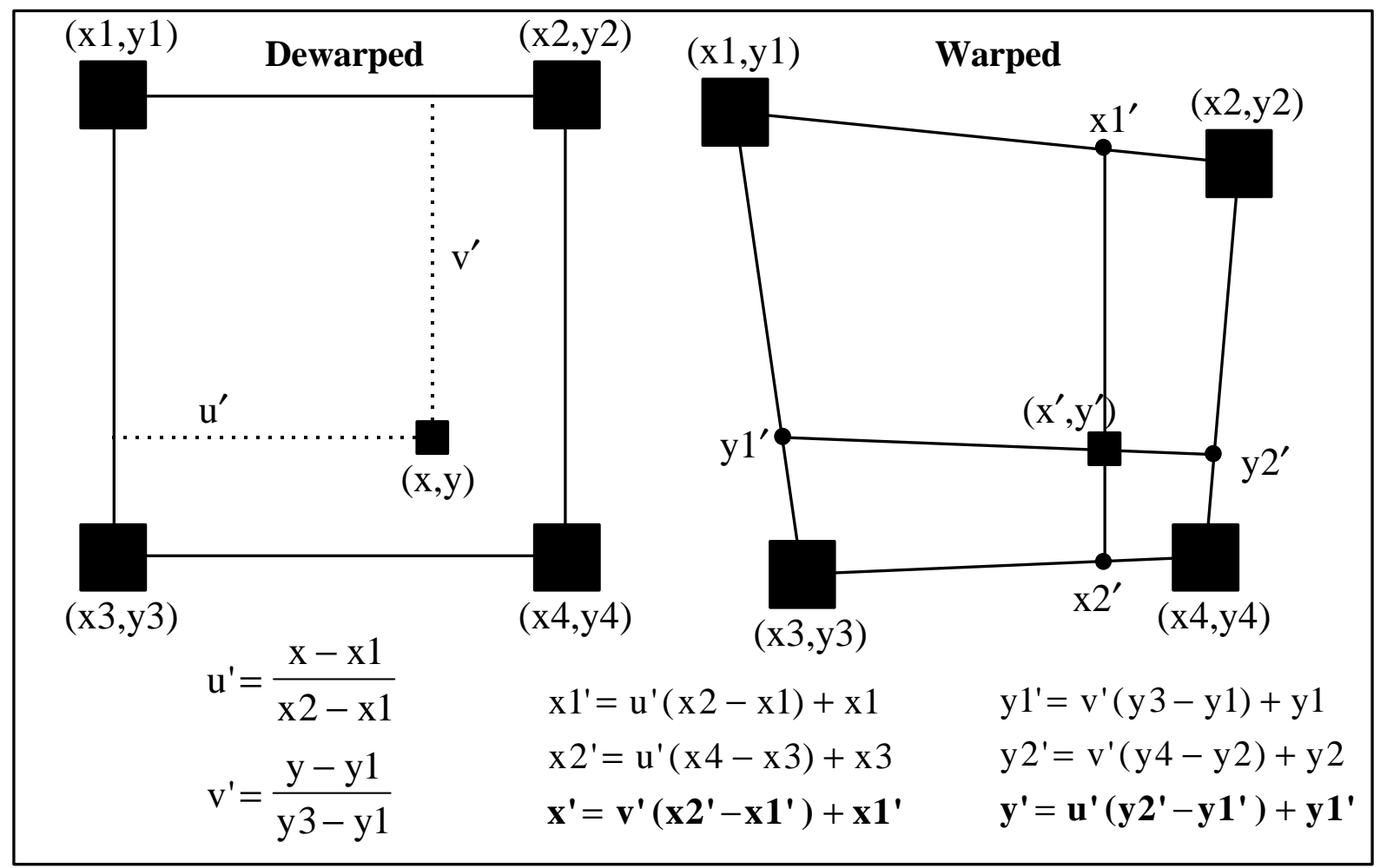

Figure 4.10. Diagram and equations used in dewarping of data images 


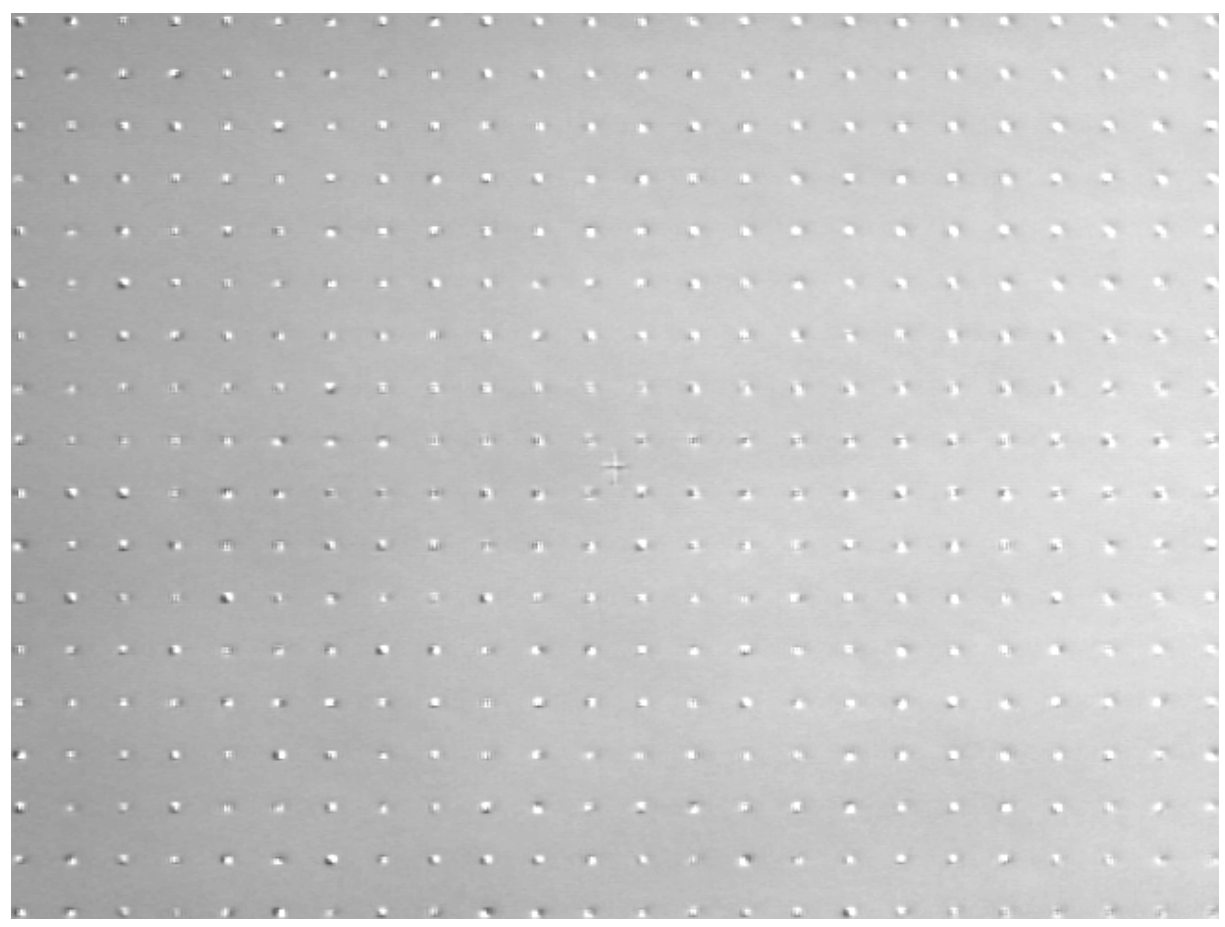

\section{Component \#1}

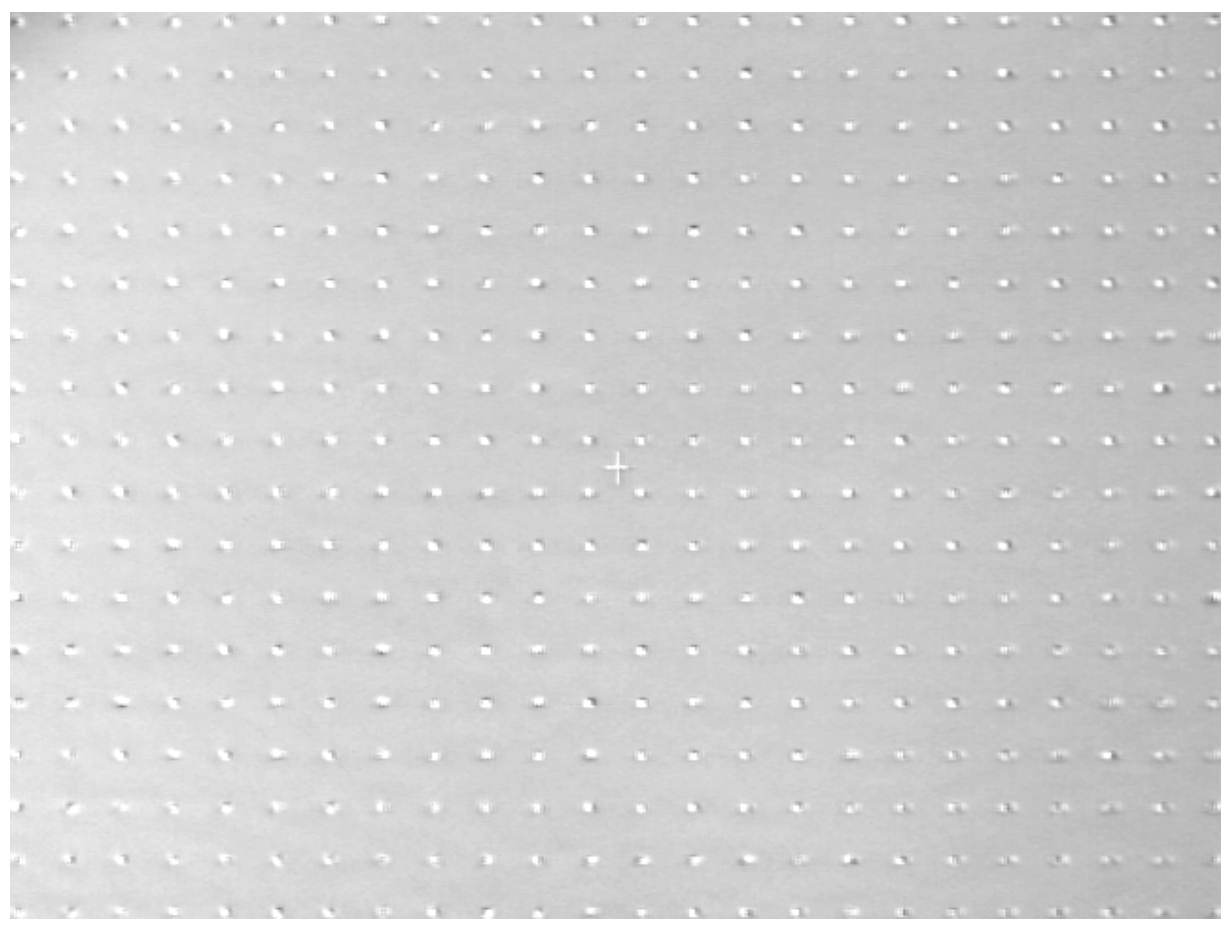

Component \#2

Figure 4.11. Overlay of signal and reference dewarped dot card images showing alignment for both Component \#1 (Top) and Component \#2 (Bottom) 


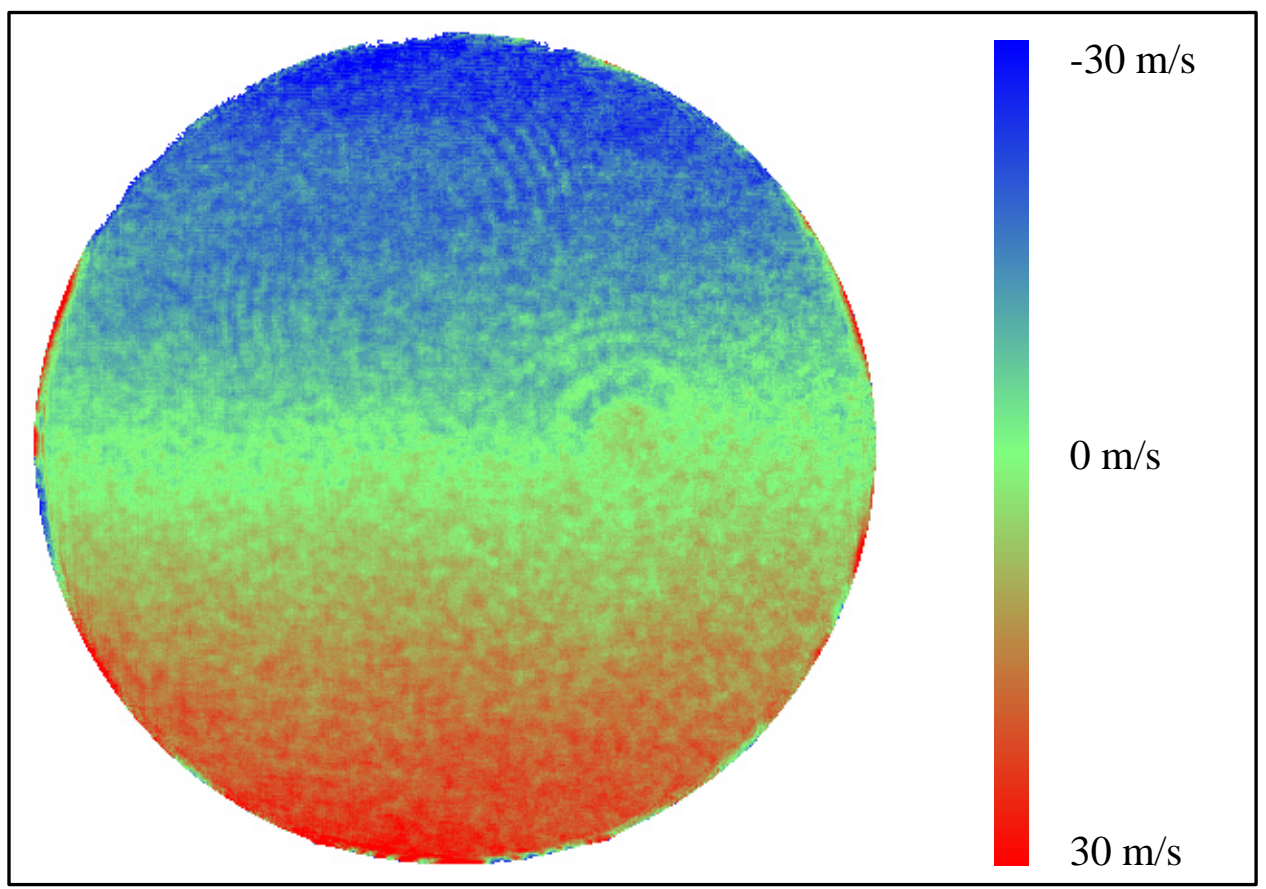

Figure 6.1. Colorized DGV Component \#1 average velocity image of rotating wheel

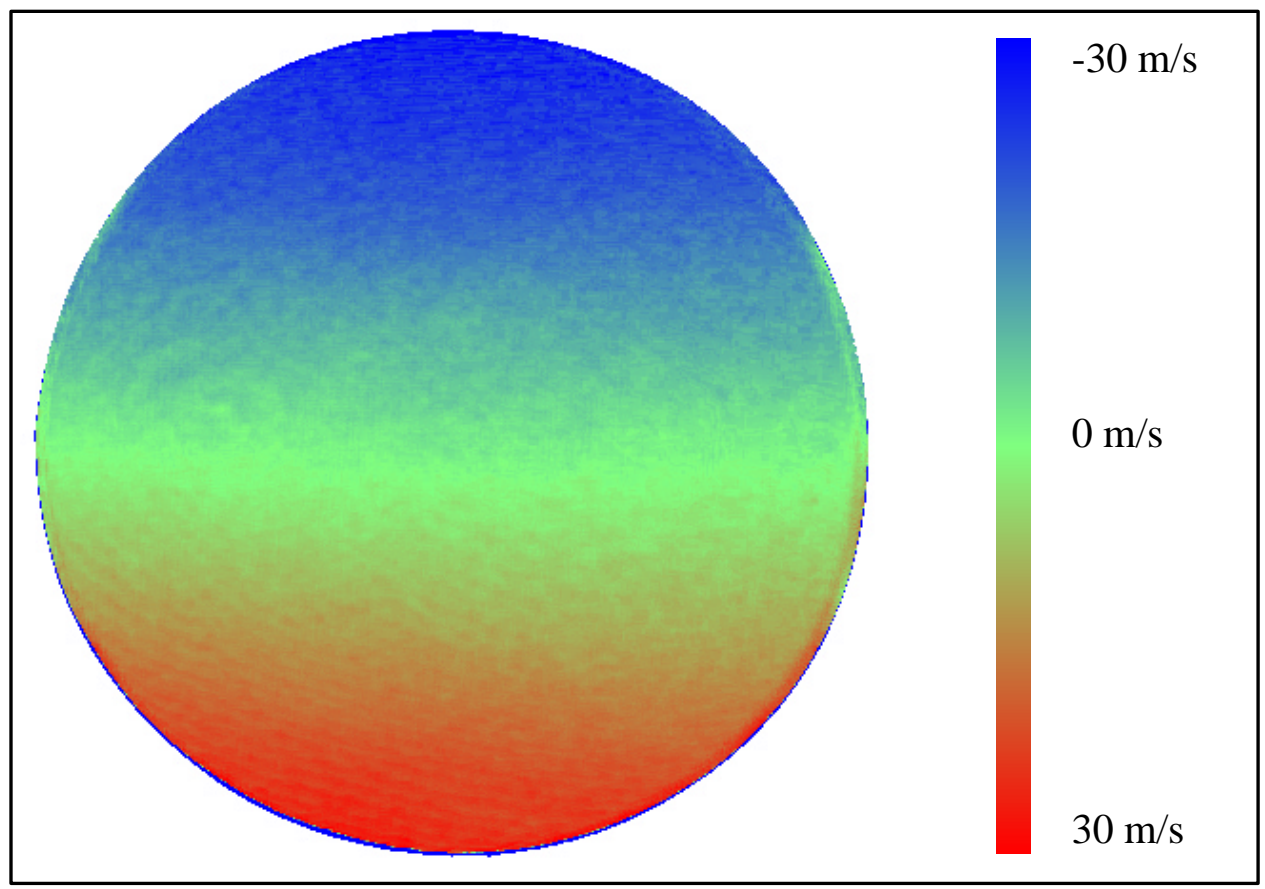

Figure 6.2. Colorized DGV Component \#2 average velocity image of rotating wheel 


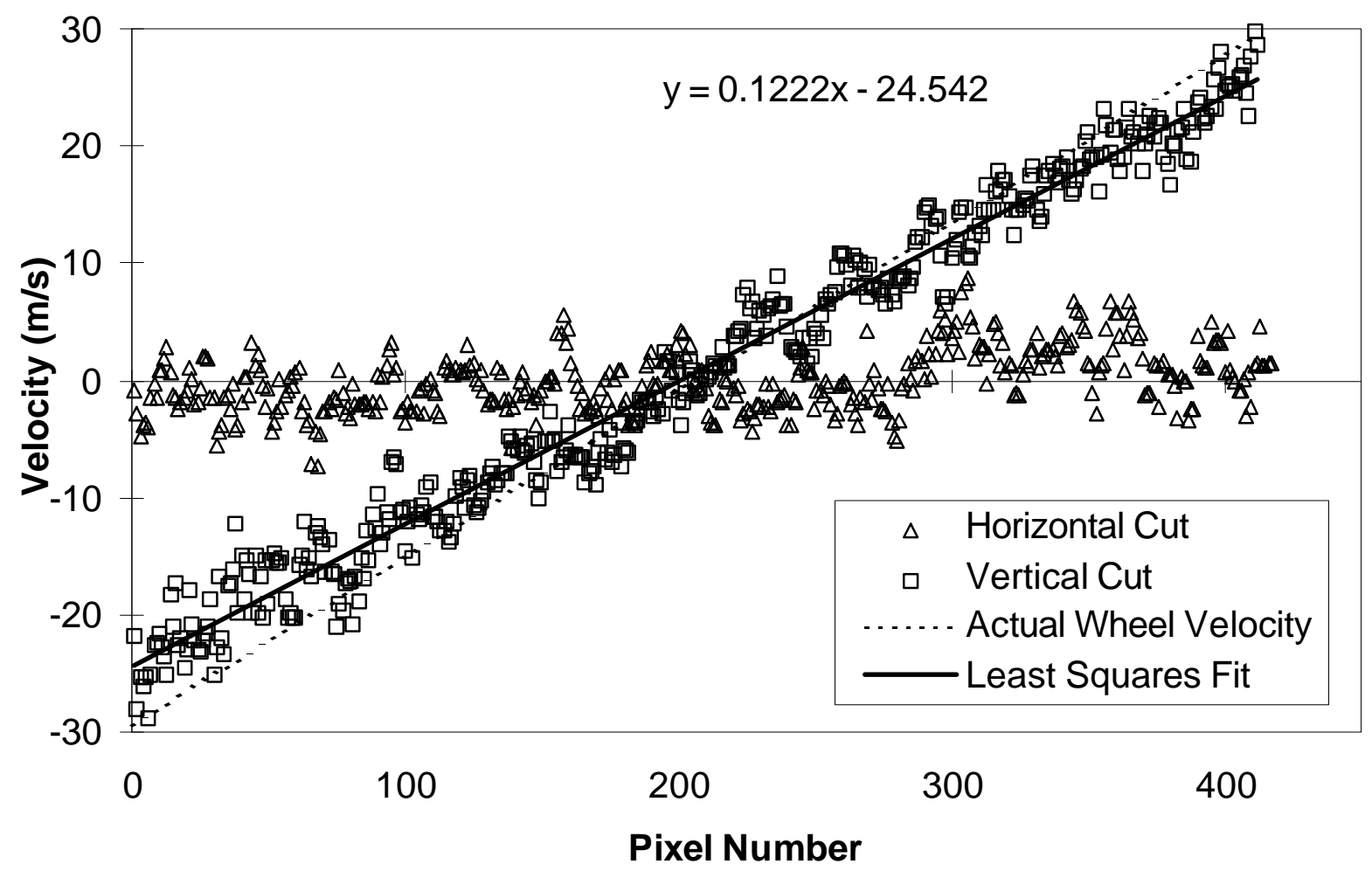

Figure 6.3. Cuts of the average DGV velocity measurement from Component 1 of a rotating wheel surface, reduced in direction of motion

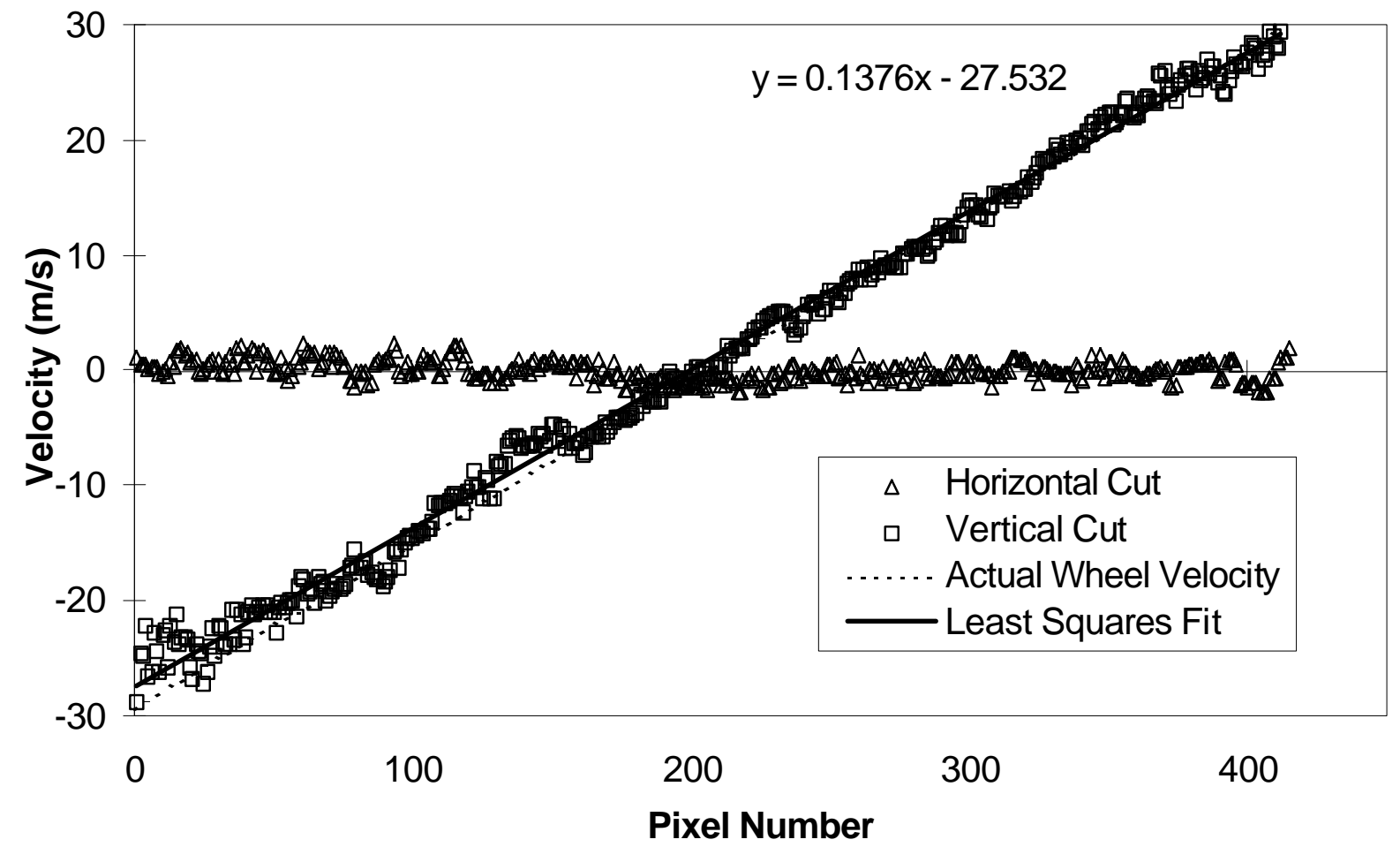

Figure 6.4. Cuts of the average DGV velocity measurement from Component 2 of a rotating wheel surface, reduced in direction of motion 


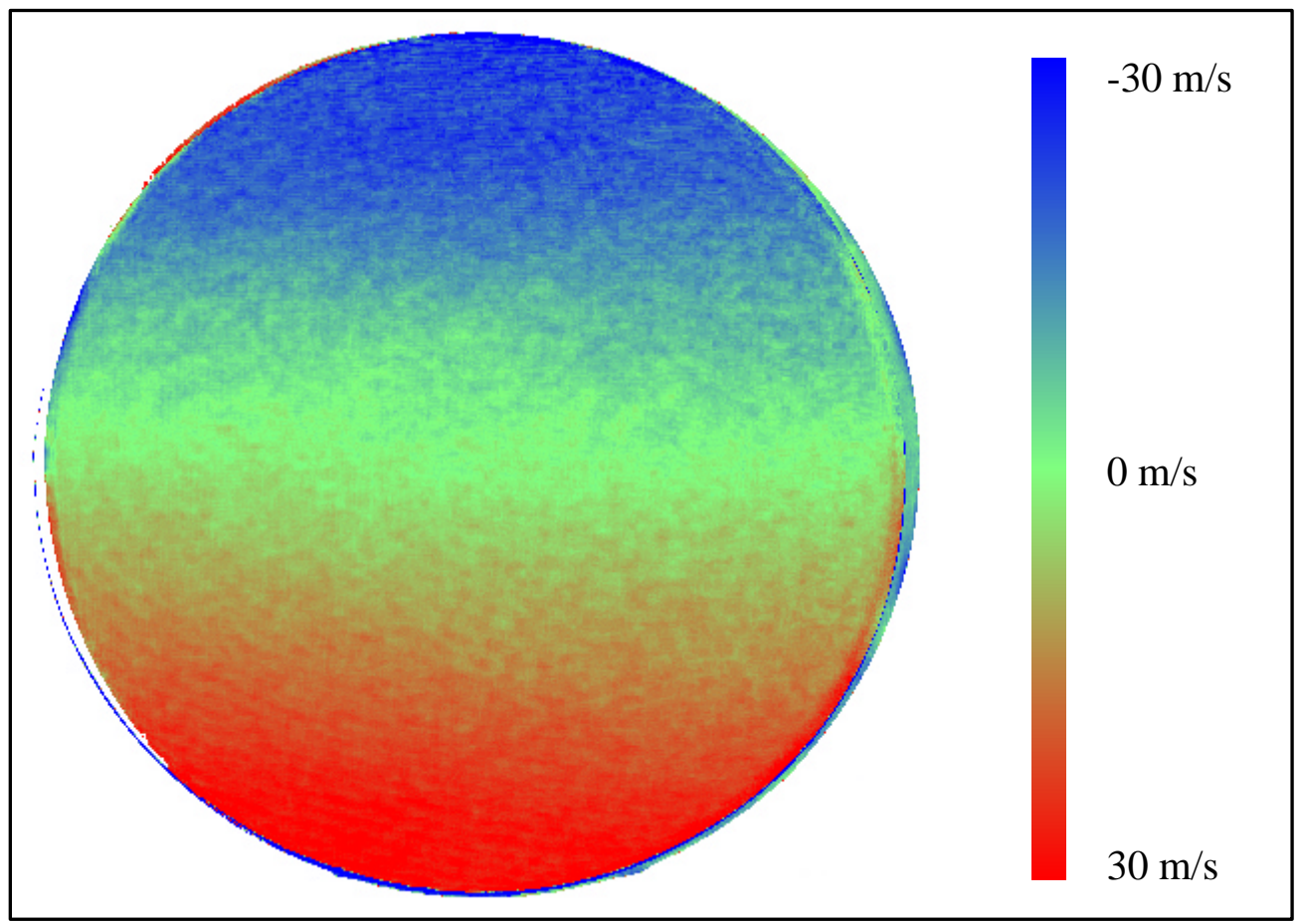

Figure 6.5. Colorized 2-component DGV tangential average velocity image of rotating wheel

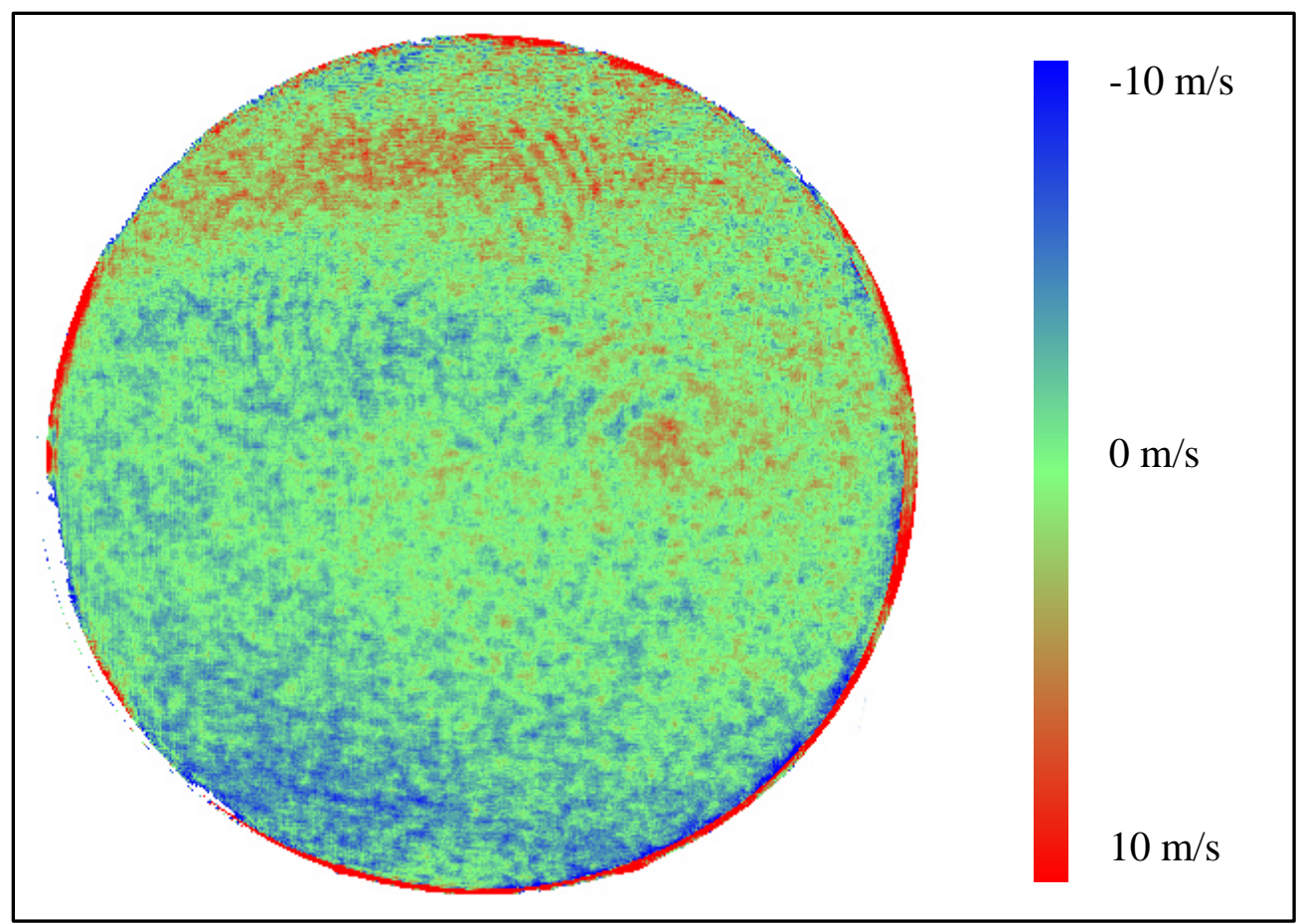

Figure 6.6. Colorized 2-component DGV normal average velocity image of rotating wheel 


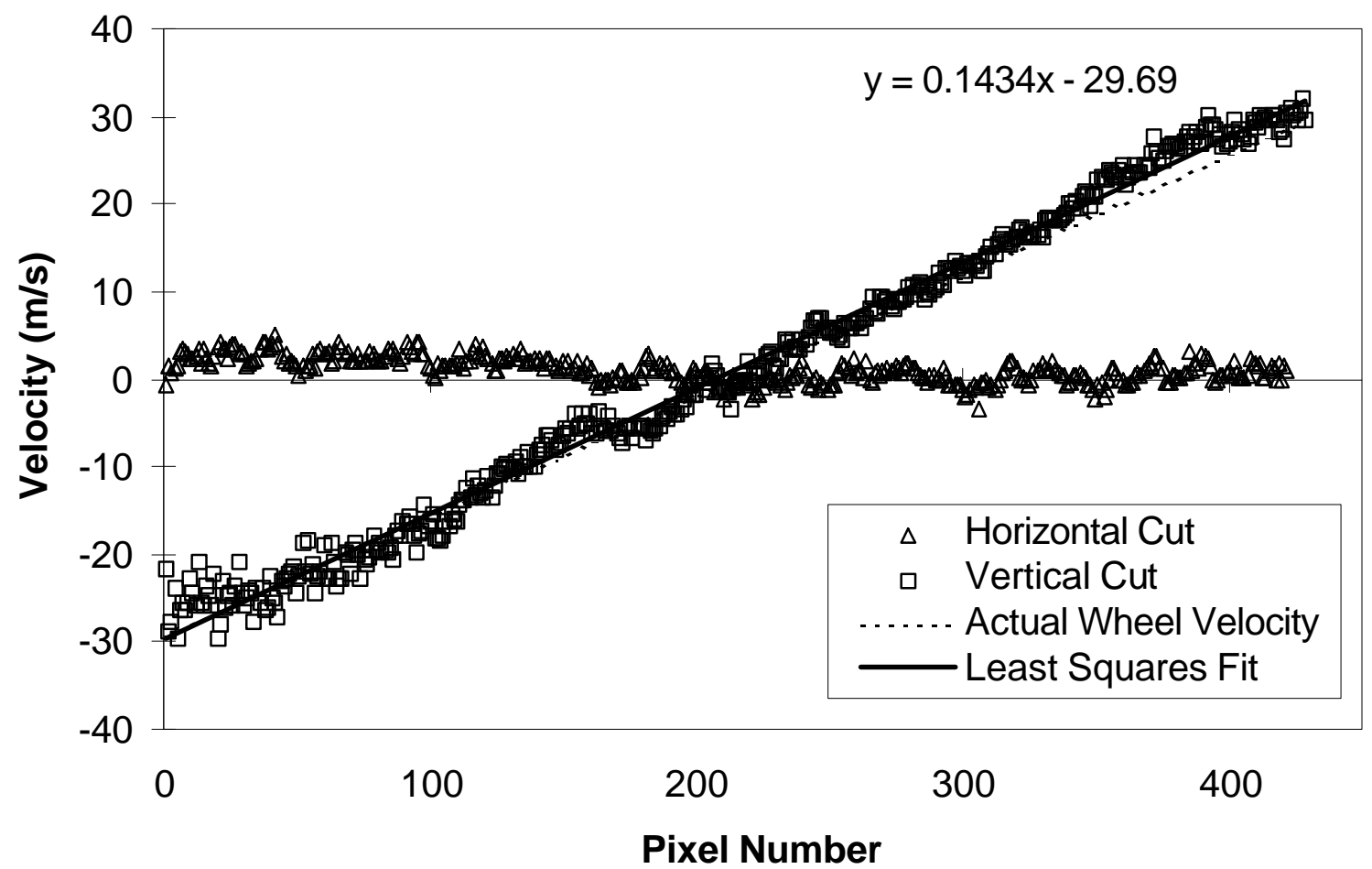

Figure 6.7. Cuts of the 2-component, average orthogonal velocity measurement parallel to the surface of a rotating wheel

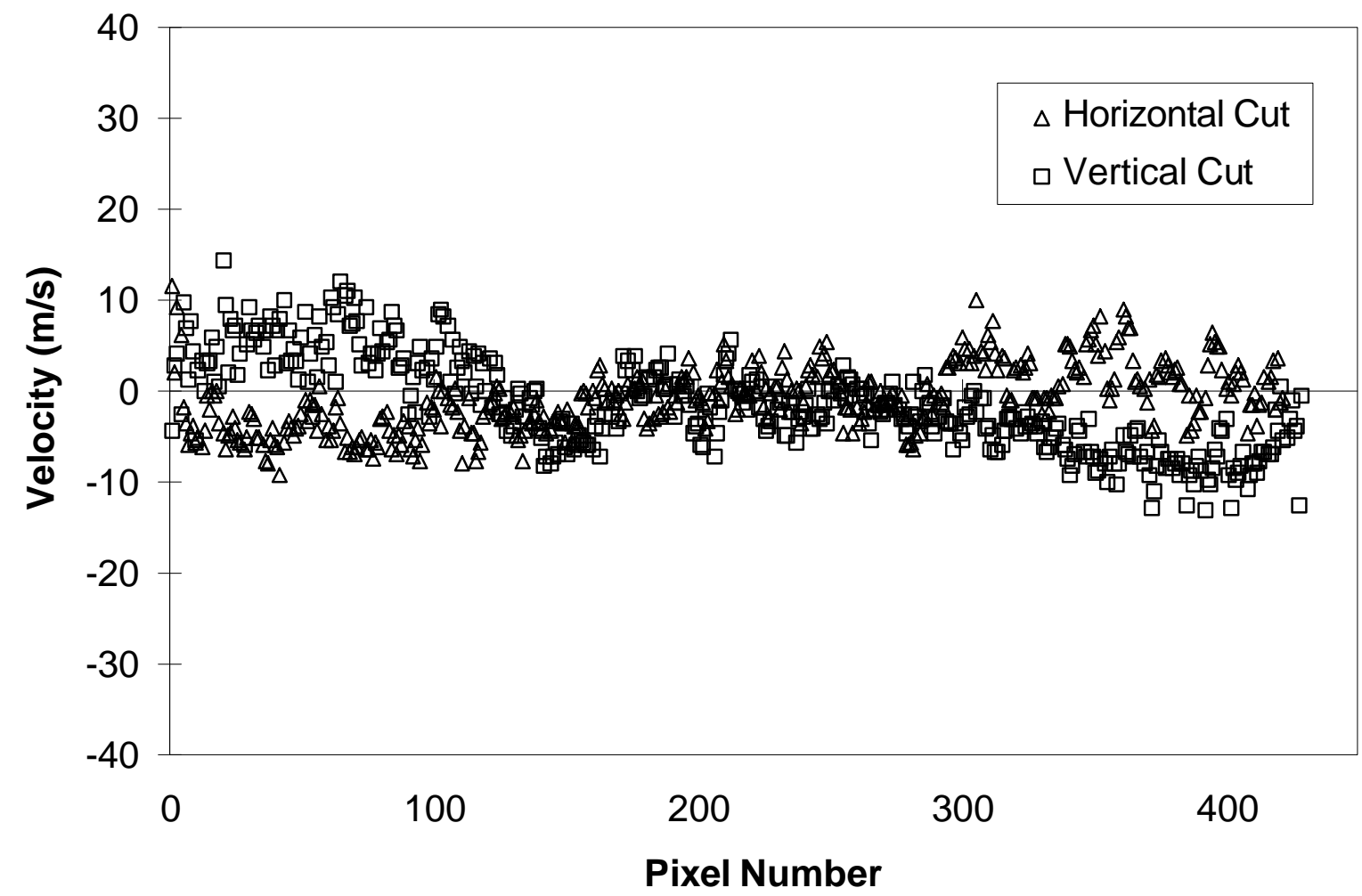

Figure 6.8. Cuts of the 2-component, average orthogonal velocity measurement normal to the surface of a rotating wheel 


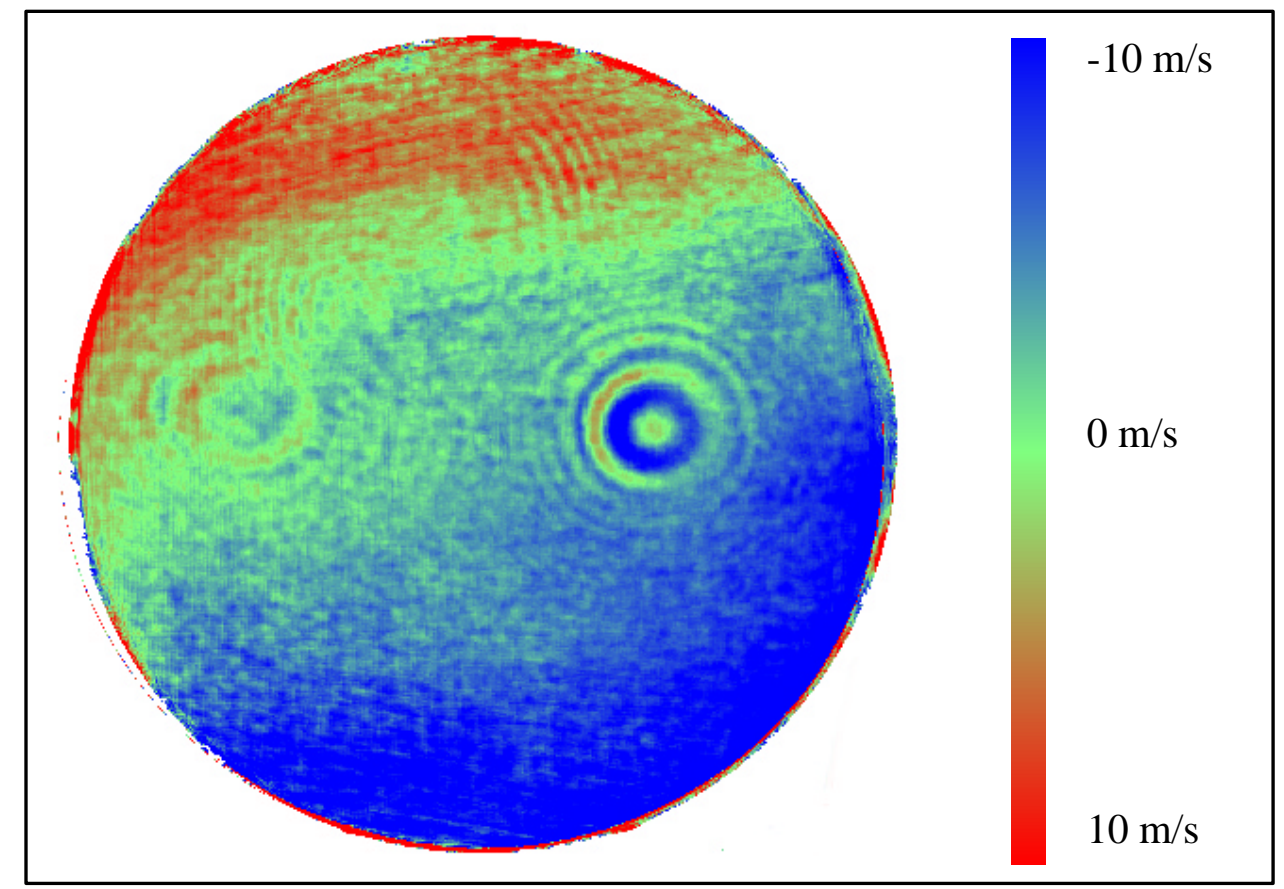

Figure 6.9. Figure 6.6 reduced without the white card correction

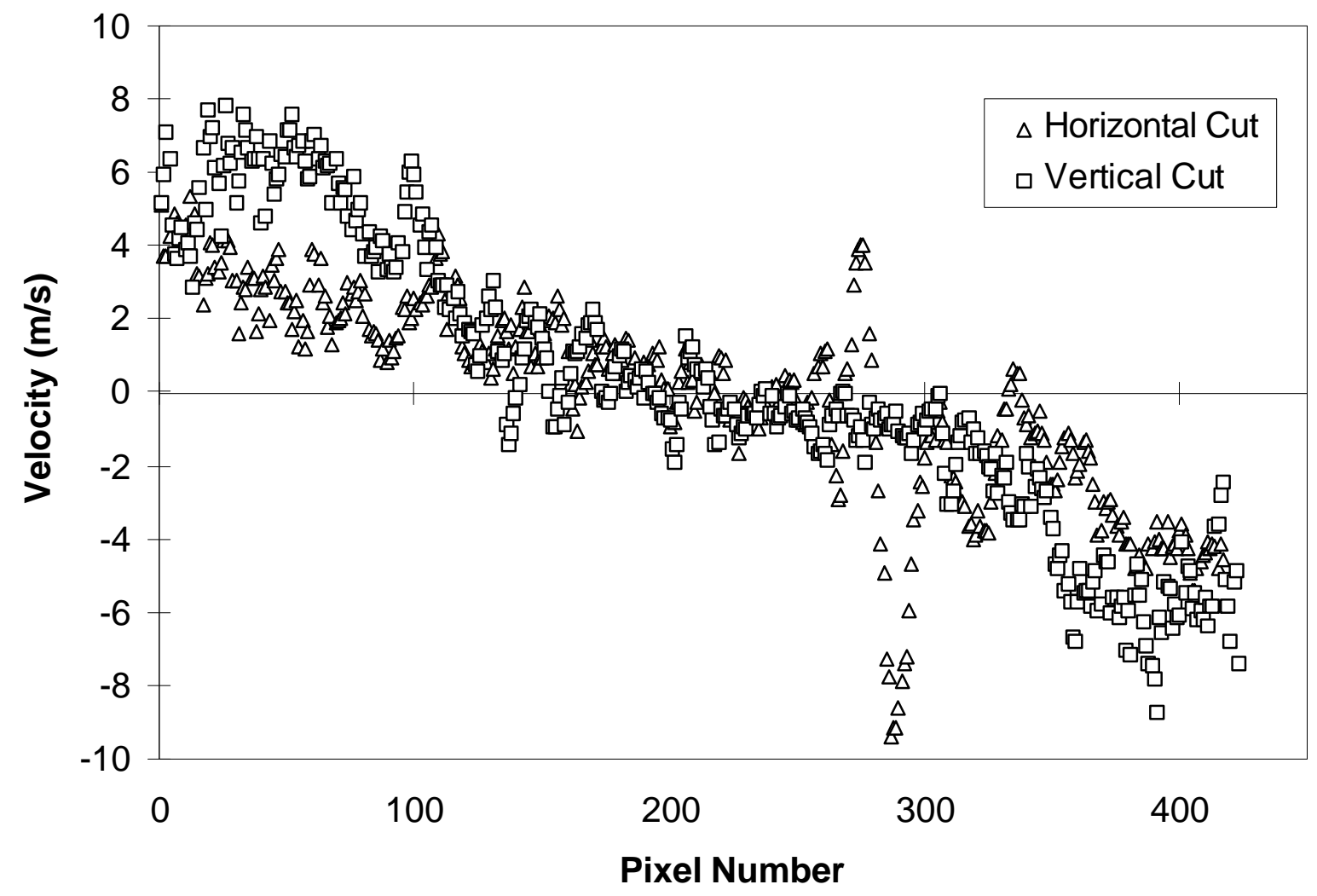

Figure 6.10. Cuts through image in Figure 6.9 showing the need for white card correction 


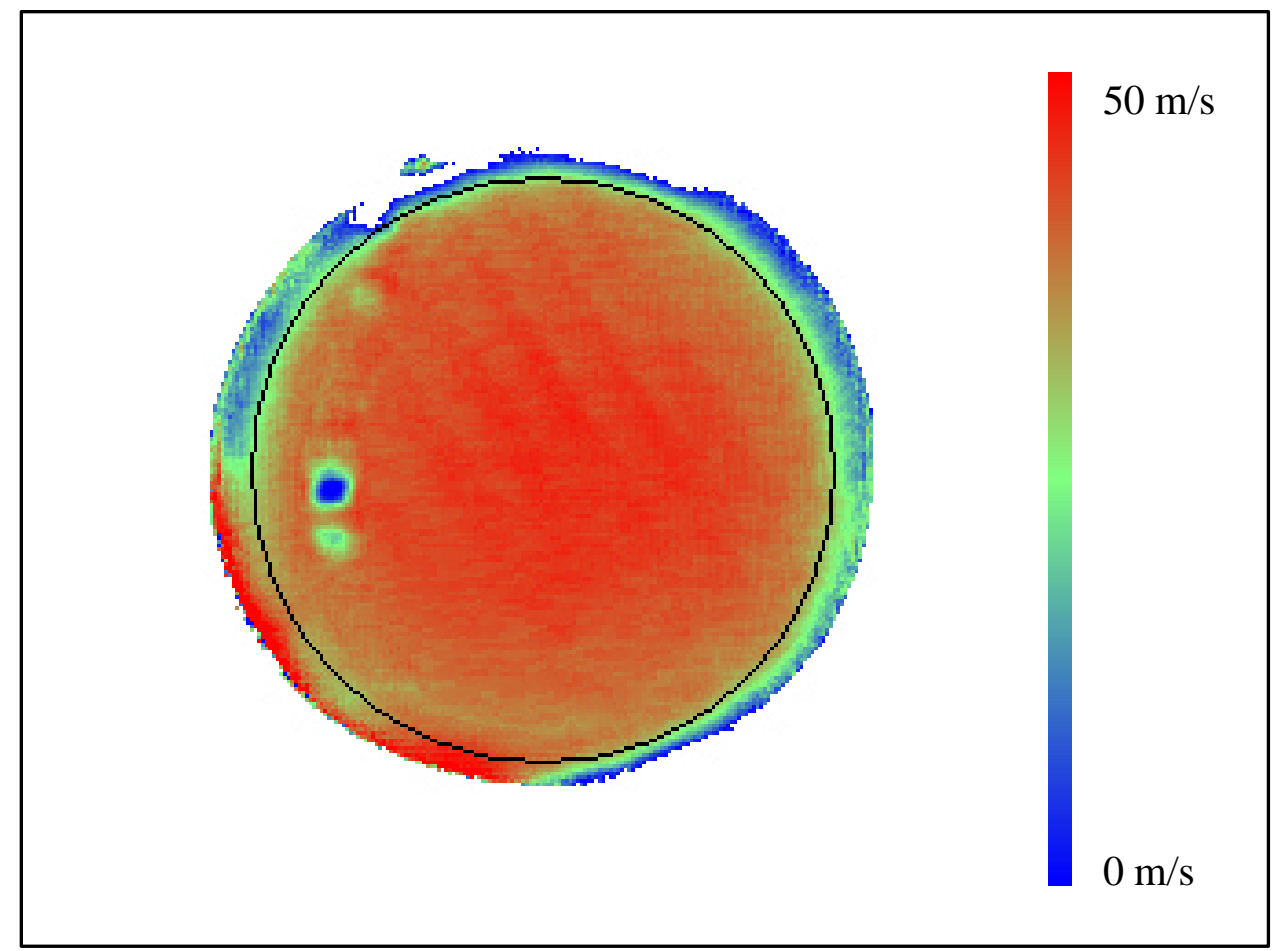

Figure 6.11. DGV averaged velocity image of fully turbulent pipe flow (Component \#2)

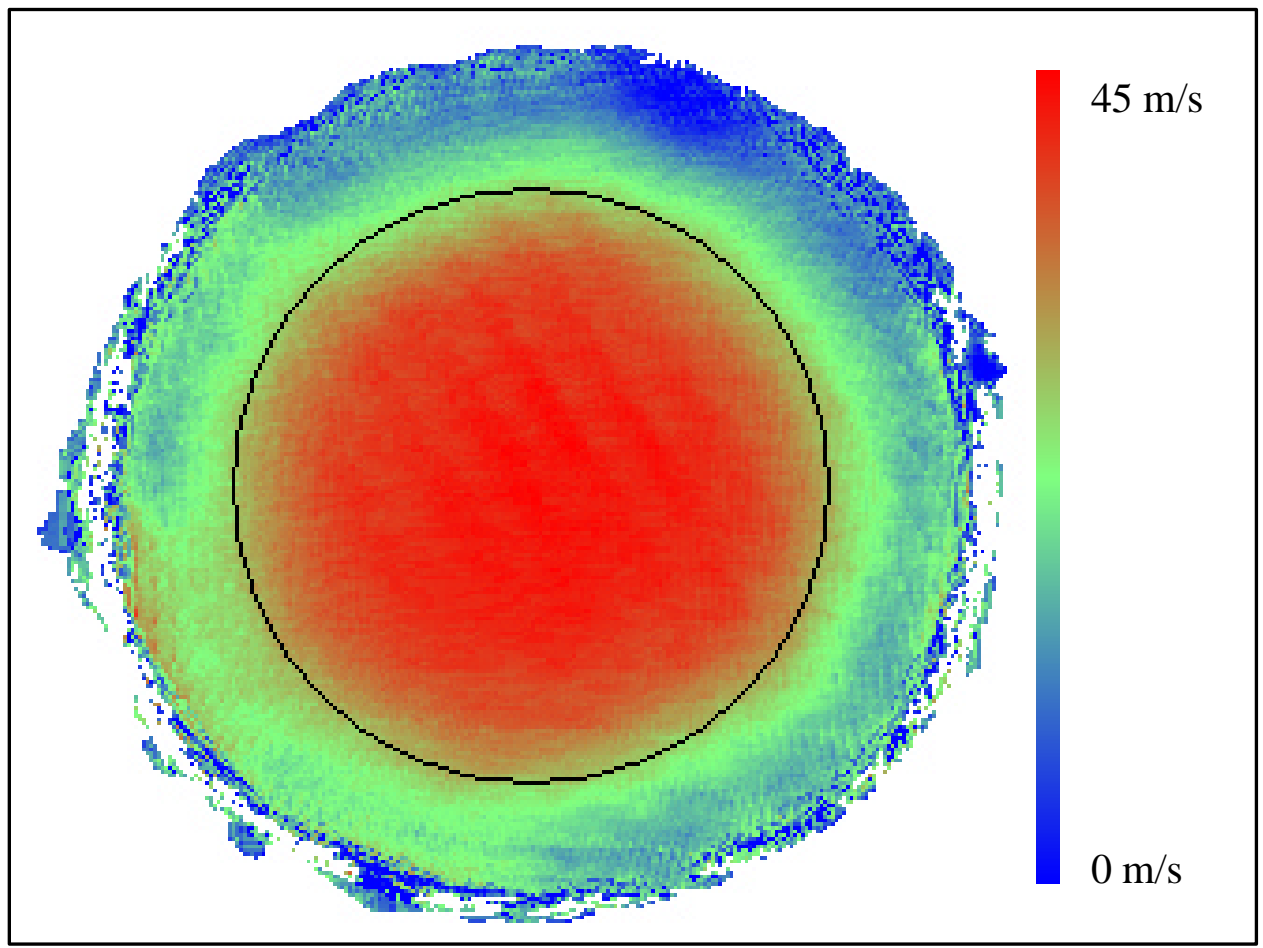

Figure 6.12. DGV averaged velocity image of turbulent jet flow, 1 diameter from exit (Component \#2) 


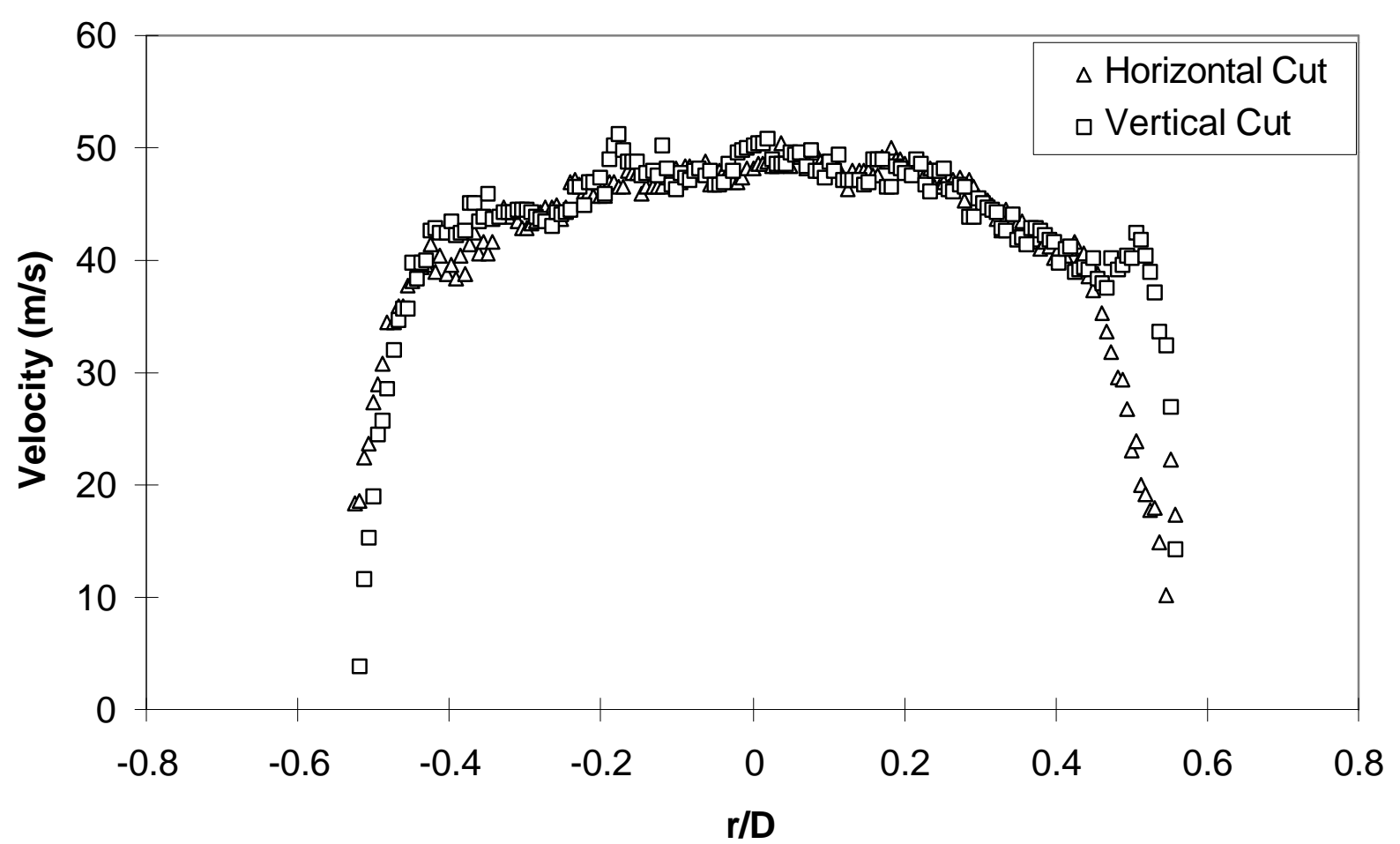

Figure 6.13. Cuts through Figure 6.11, Component \#2 DGV fully turbulent pipe flow velocity measurements

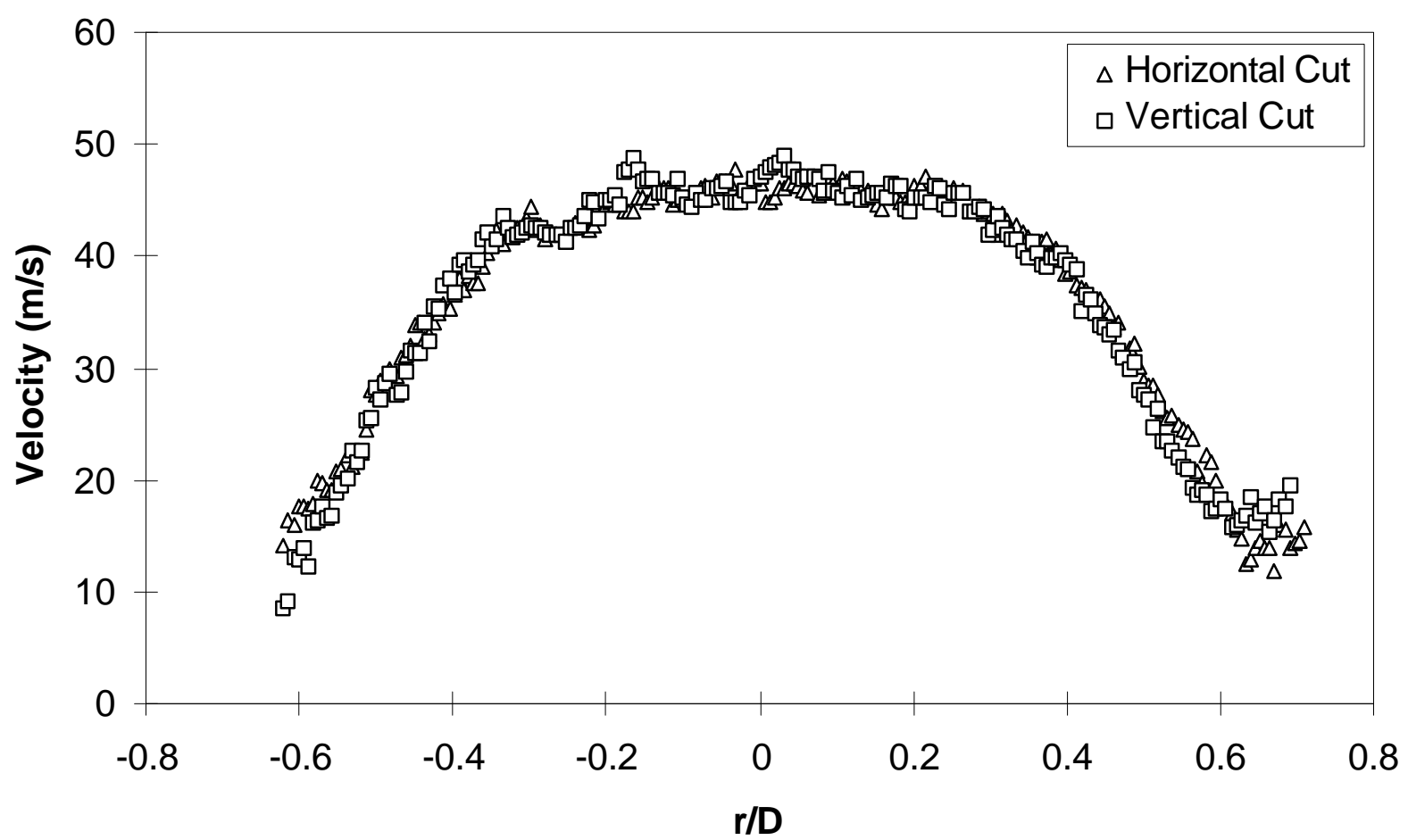

Figure 6.14. Cuts through Figure 6.12, Component \#2 DGV jet velocity measurements 1 diameter from the exit 


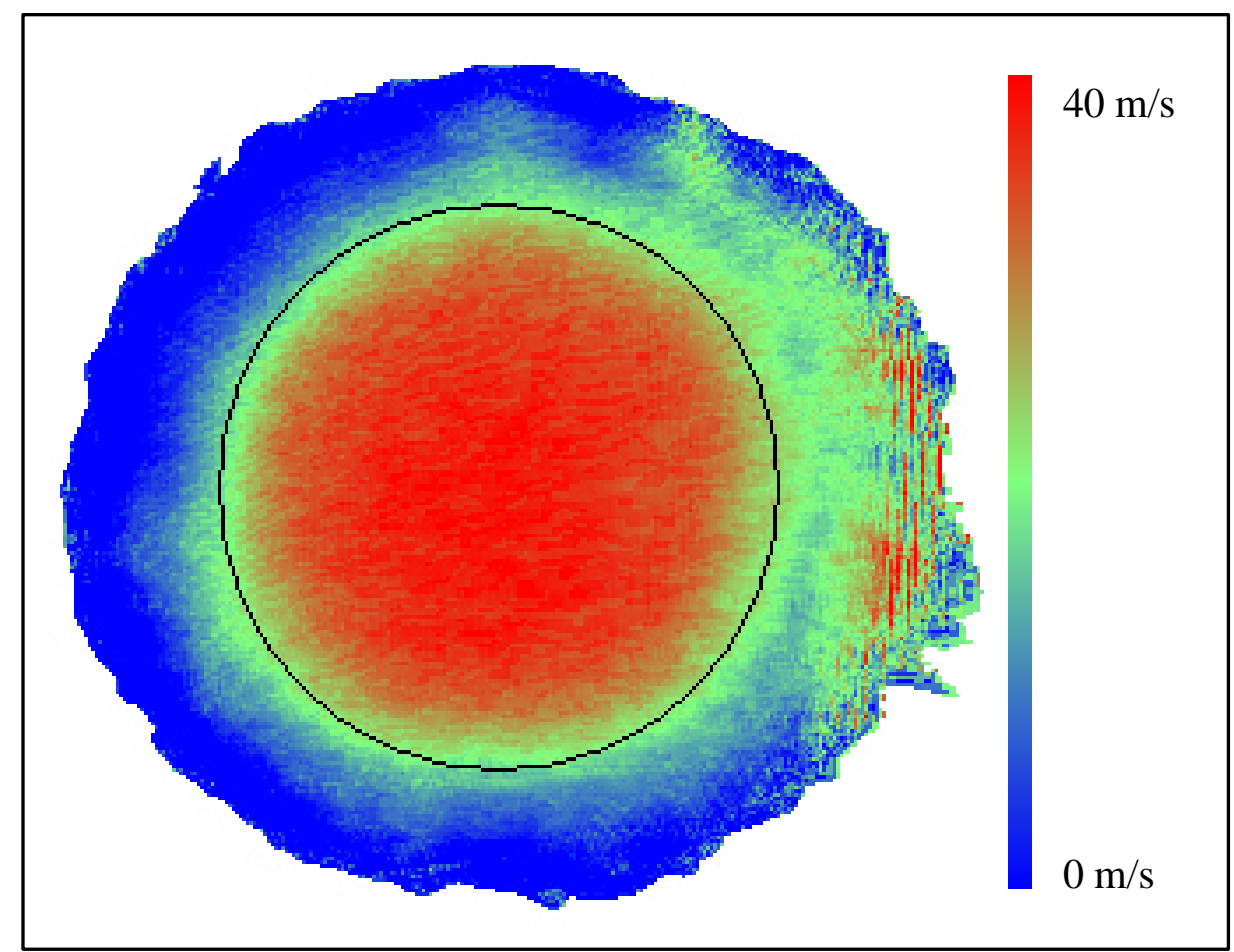

Figure 6.15. DGV averaged velocity image of turbulent jet flow, 1 diameters from exit (Component \#1-- run 2)

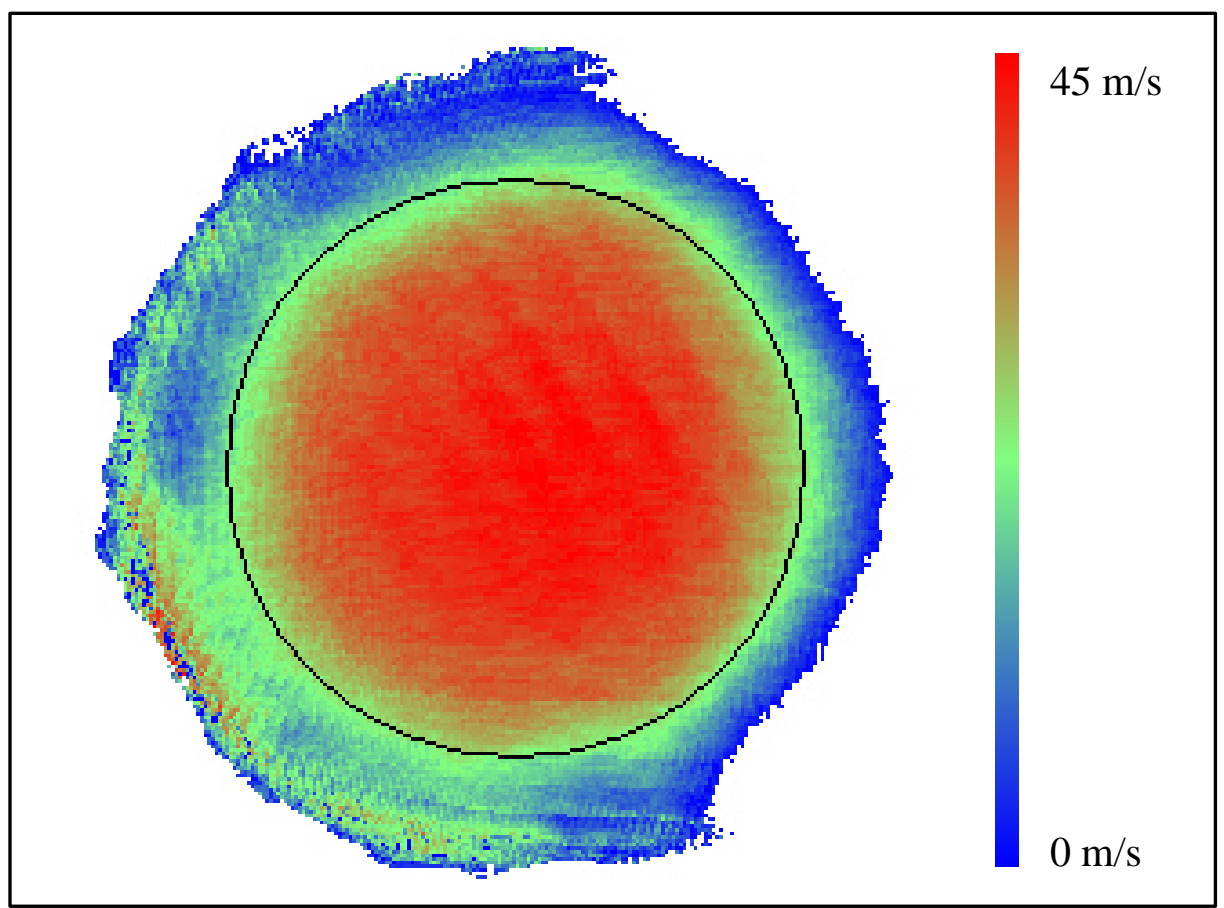

Figure 6.16. DGV averaged velocity image of turbulent jet flow, 1 diameters from exit (Component \#2 -- run 2) 


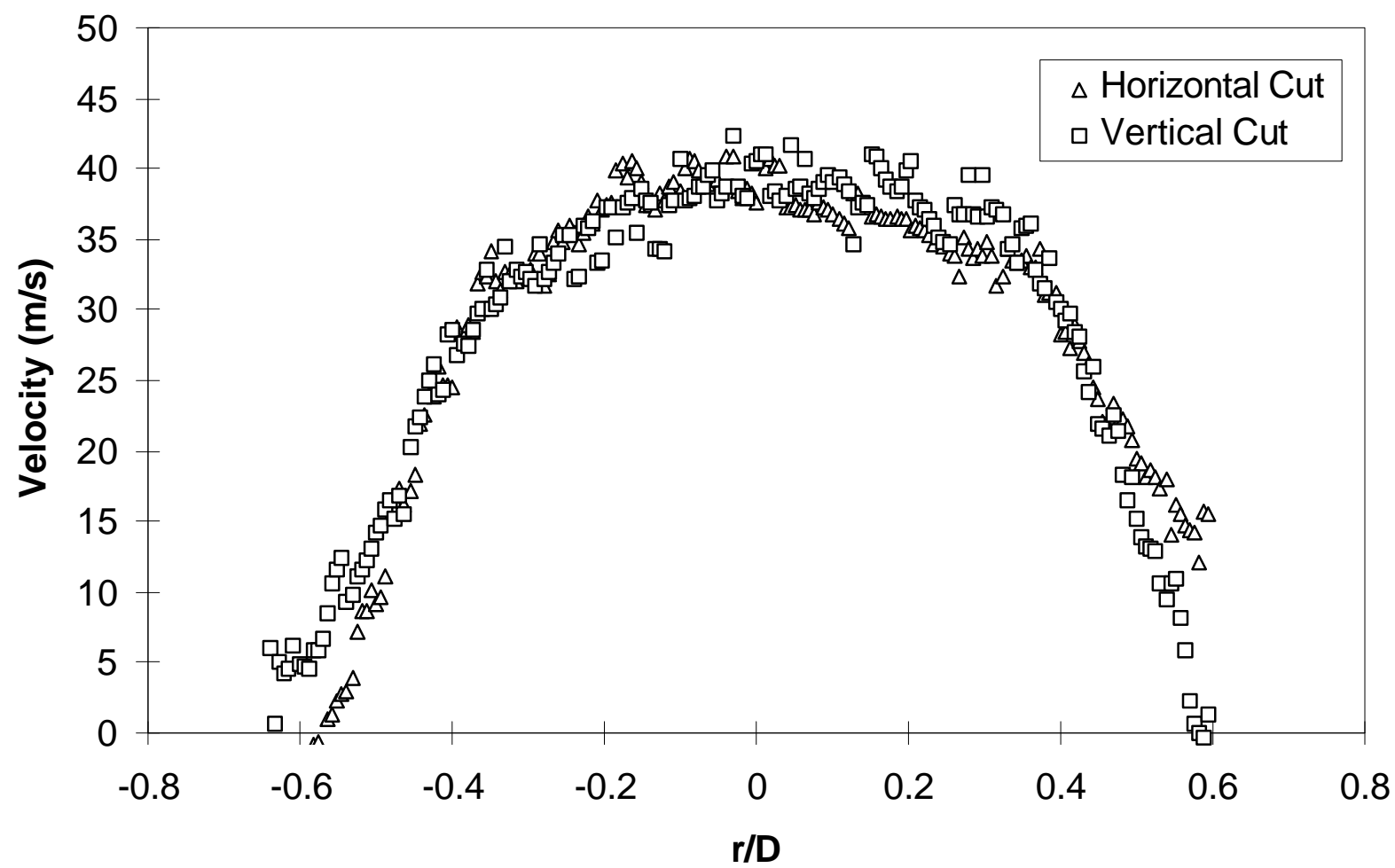

Figure 6.17. Cuts through Figure 6.15, Component \#1 DGV jet velocity measurements 1 diameter from the exit

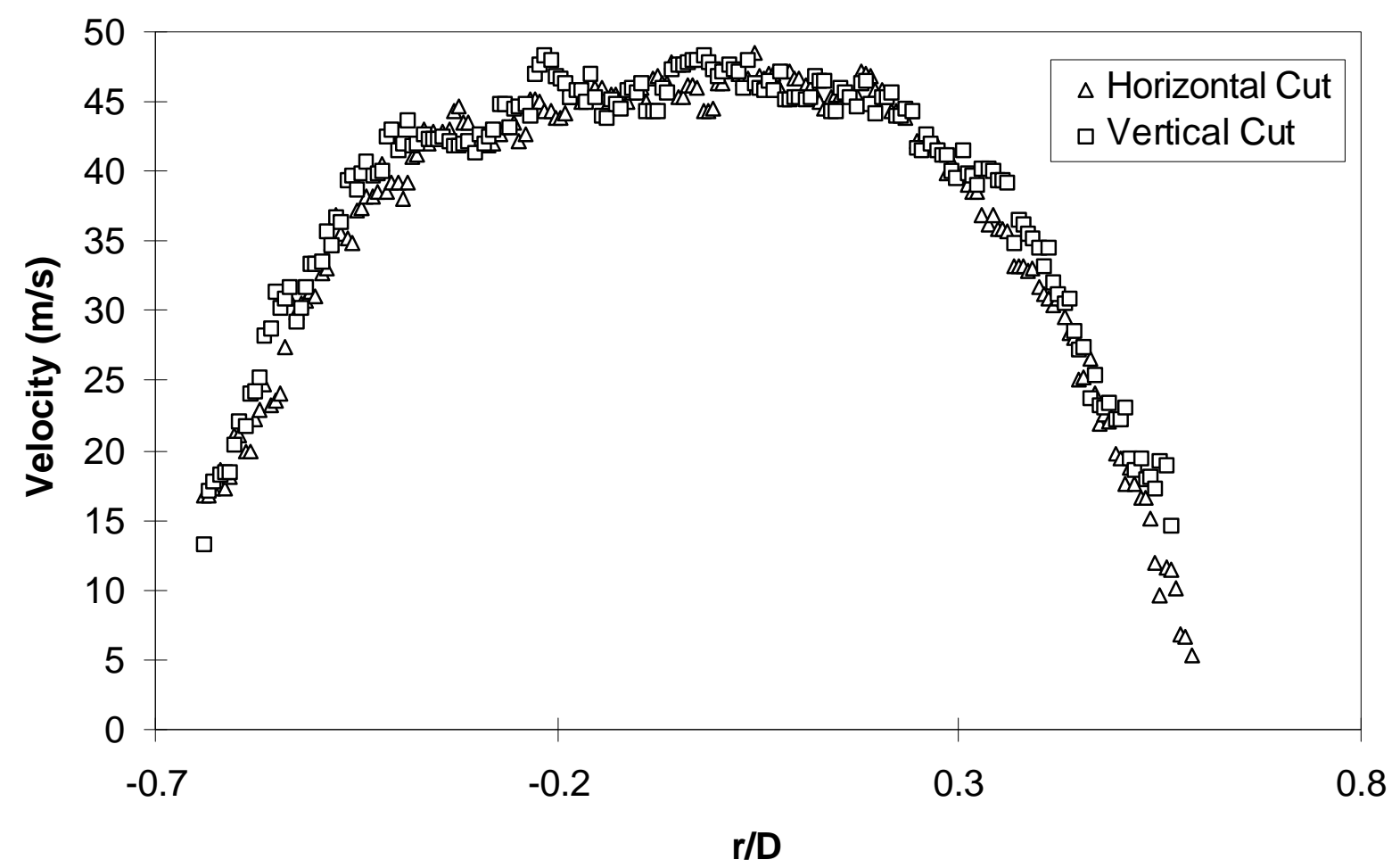

Figure 6.18. Cuts through Figure 6.16, Component \#2 DGV jet velocity measurements 1 diameter from the exit 


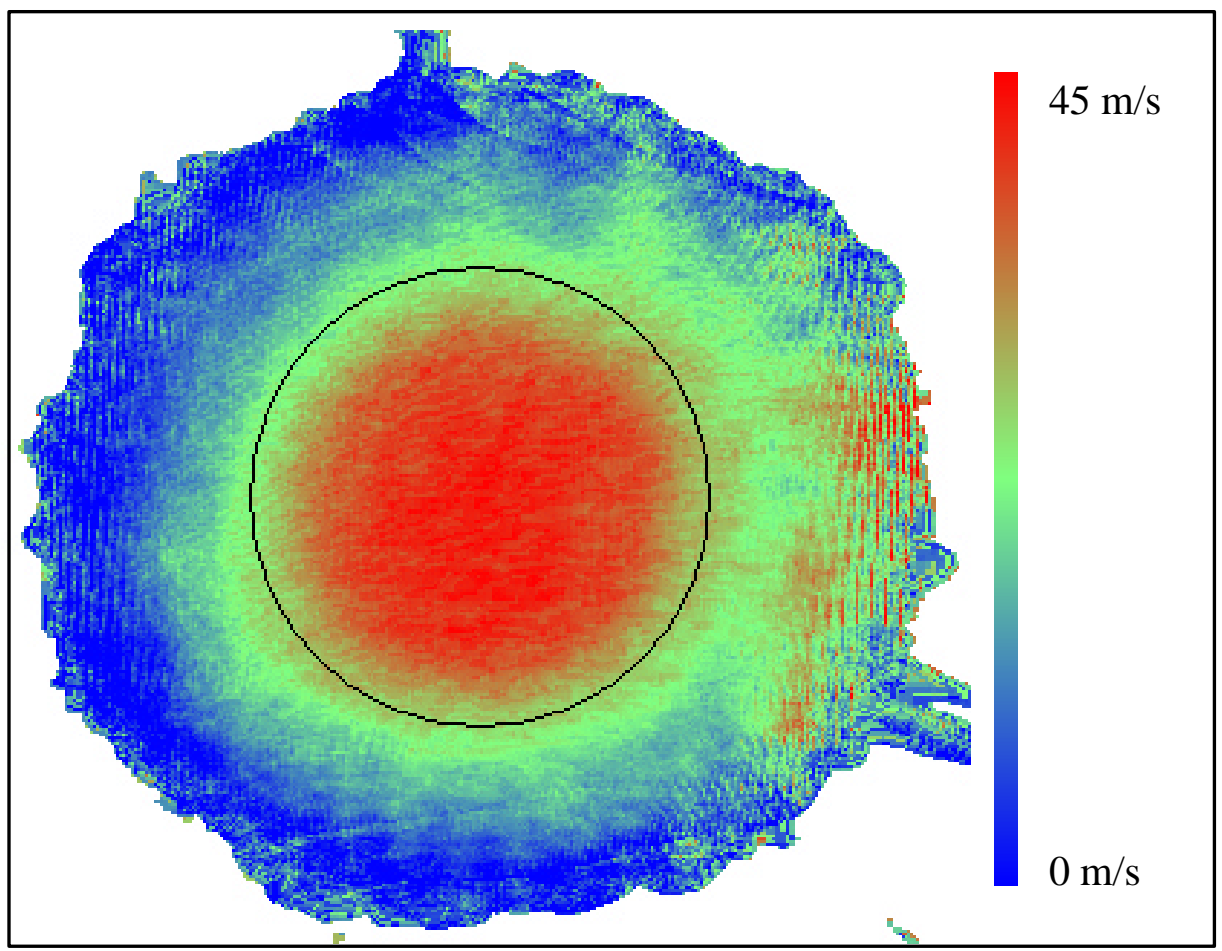

Figure 6.19. DGV averaged velocity image of turbulent jet flow, 2 diameters from exit (Component \#1)

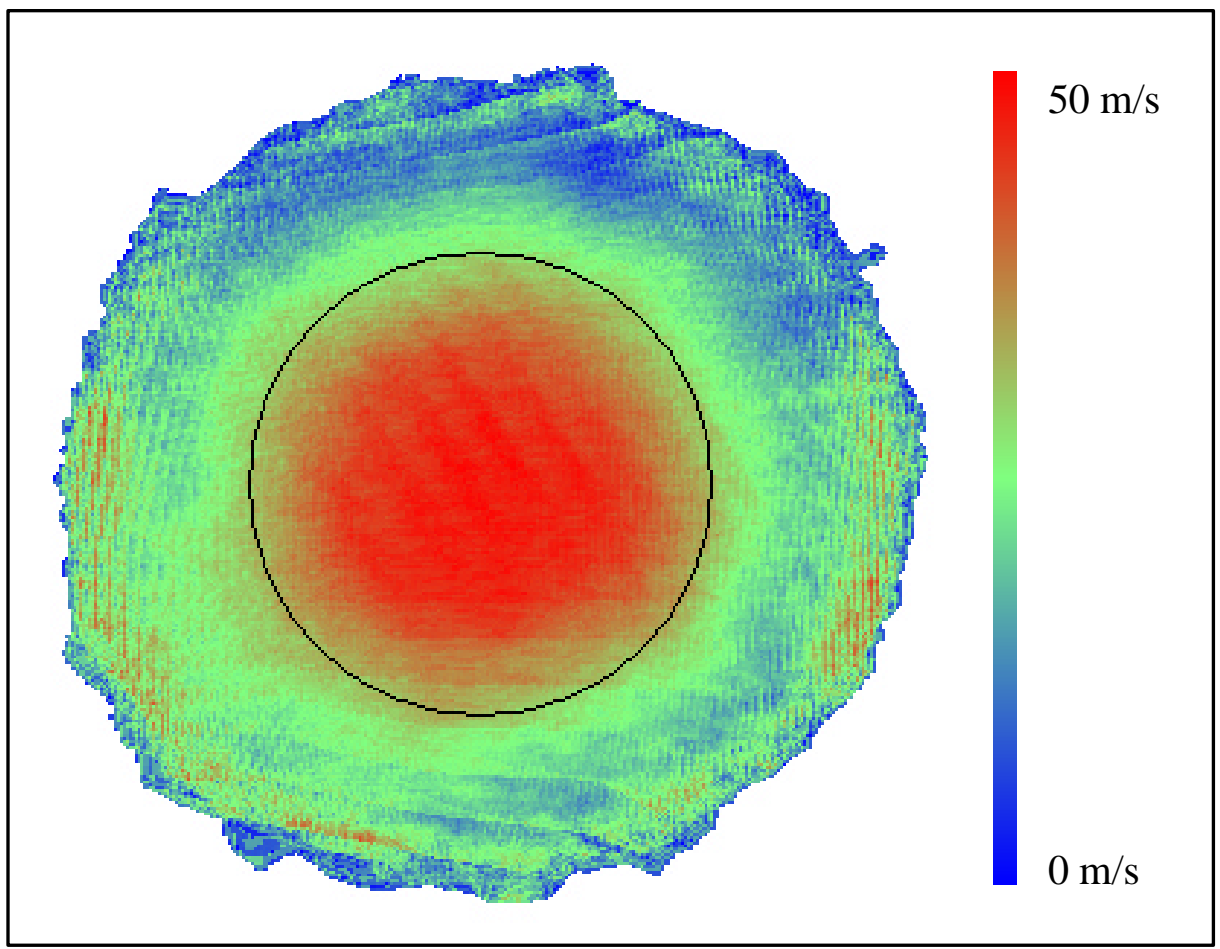

Figure 6.20. DGV averaged velocity image of turbulent jet flow, 2 diameters from exit (Component \#2) 


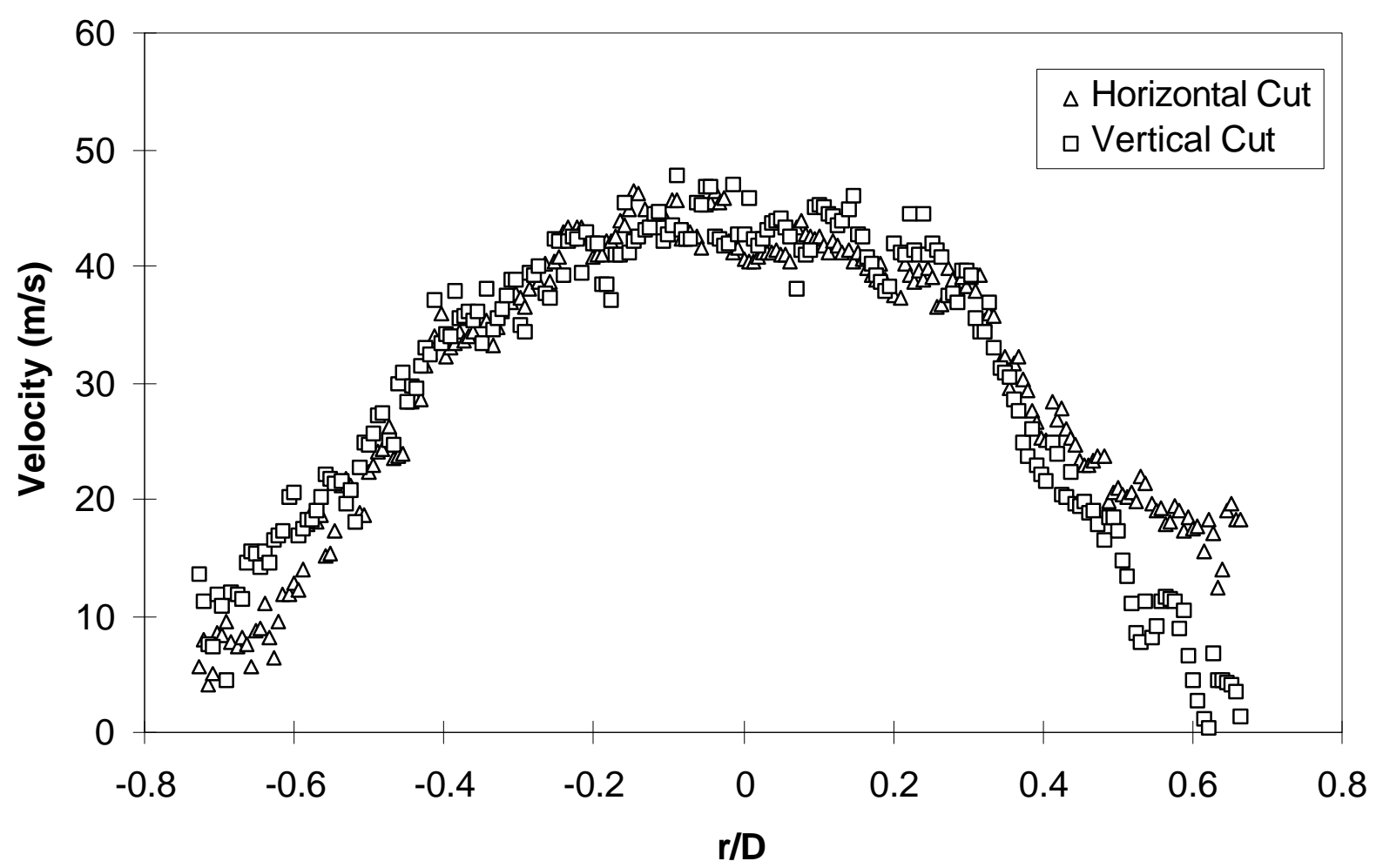

Figure 6.21. Cuts through Figure 6.19, Component \#1 DGV jet velocity measurements 2 diameters from the exit

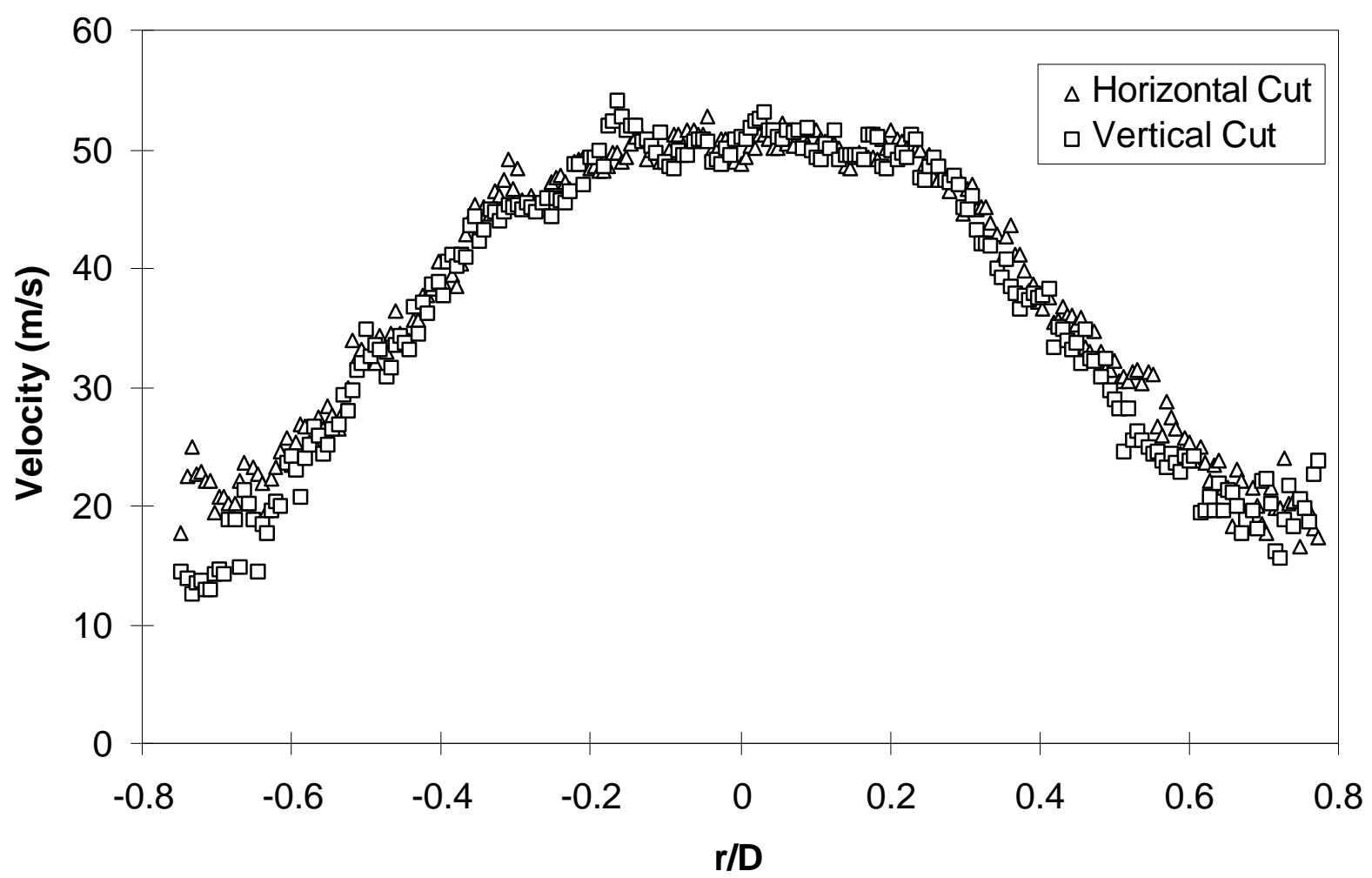

Figure 6.22. Cuts through Figure 6.20, Component \#2 DGV jet velocity measurements 2 diameters from the exit 


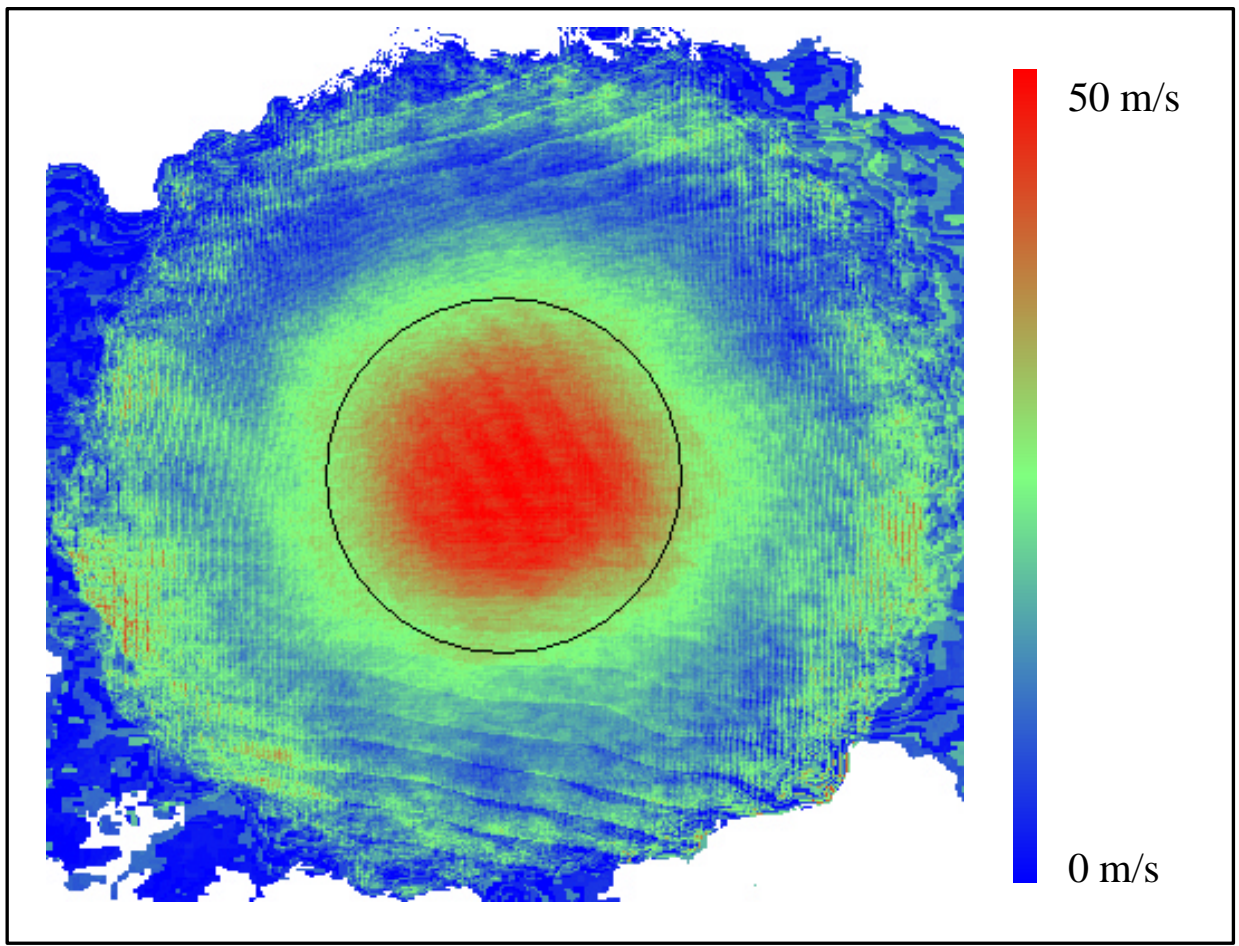

Figure 6.23. DGV averaged velocity image of a turbulent jet, 4 diameters from the exit (Component \#2)

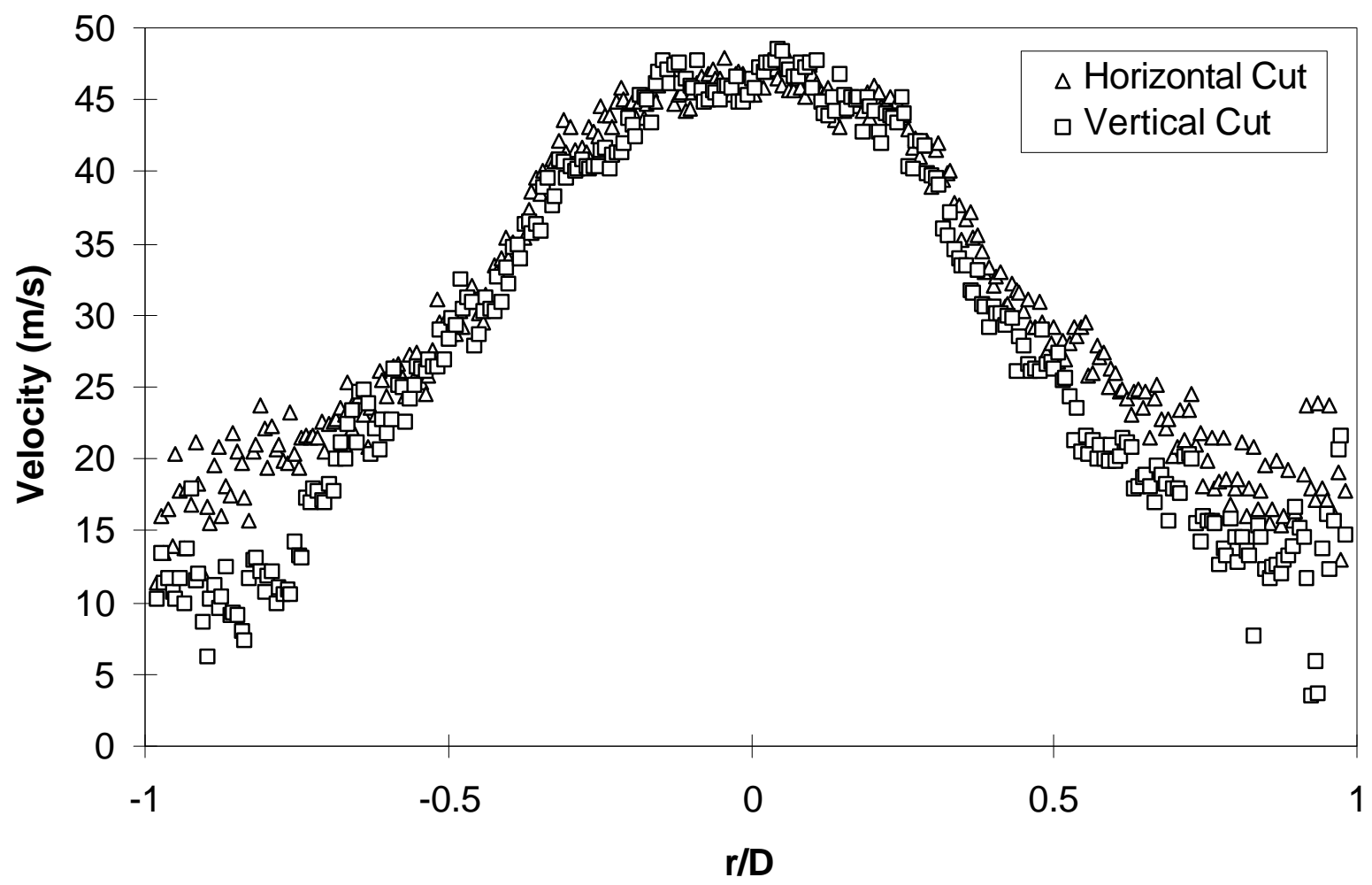

Figure 6.24. Cuts through Figure 6.23, Component \#2 DGV jet velocity measurements 4 diameters from the exit 


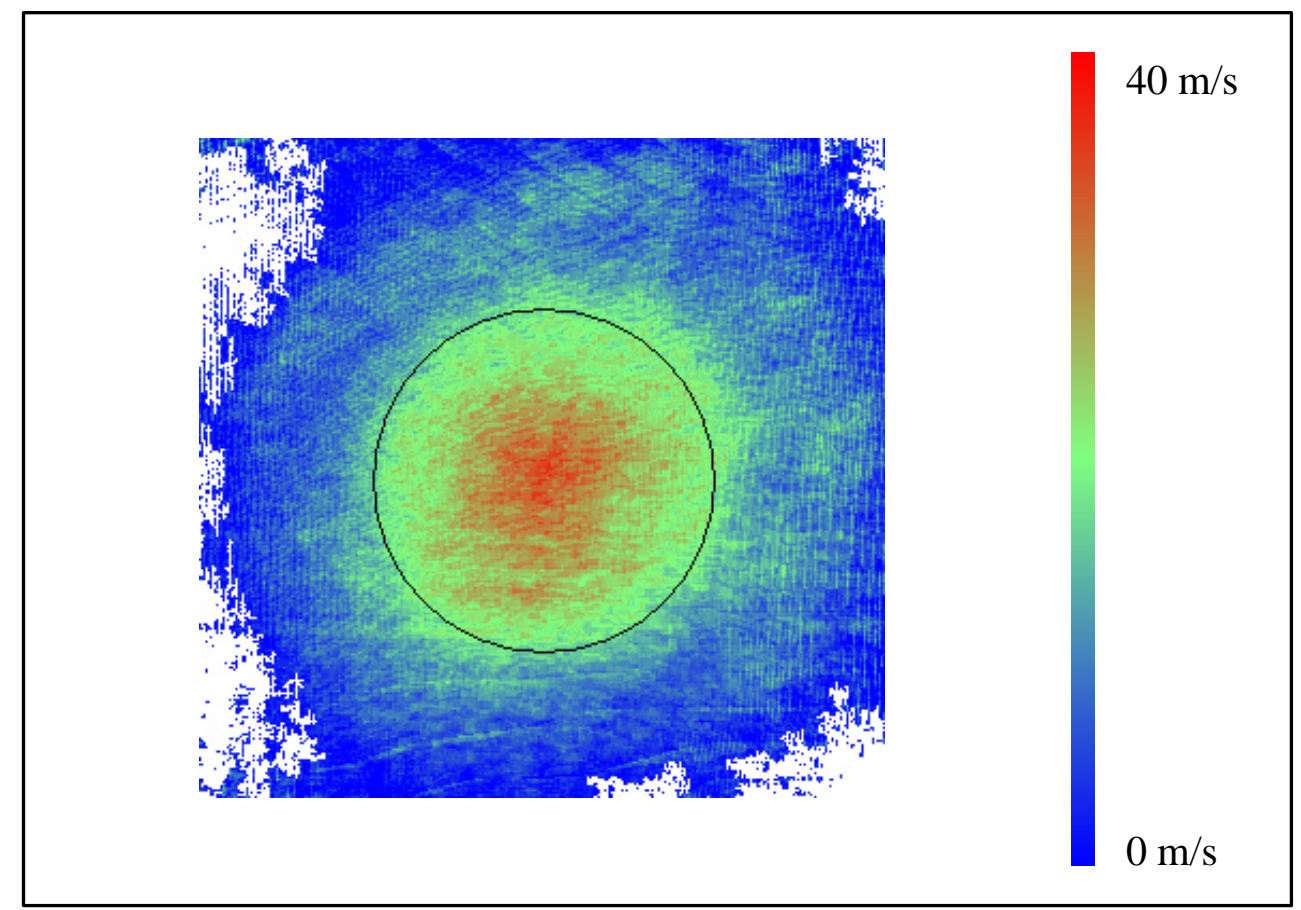

Figure 6.25. DGV velocity image of turbulent jet flow, 6 diameters from exit (Component \#1)

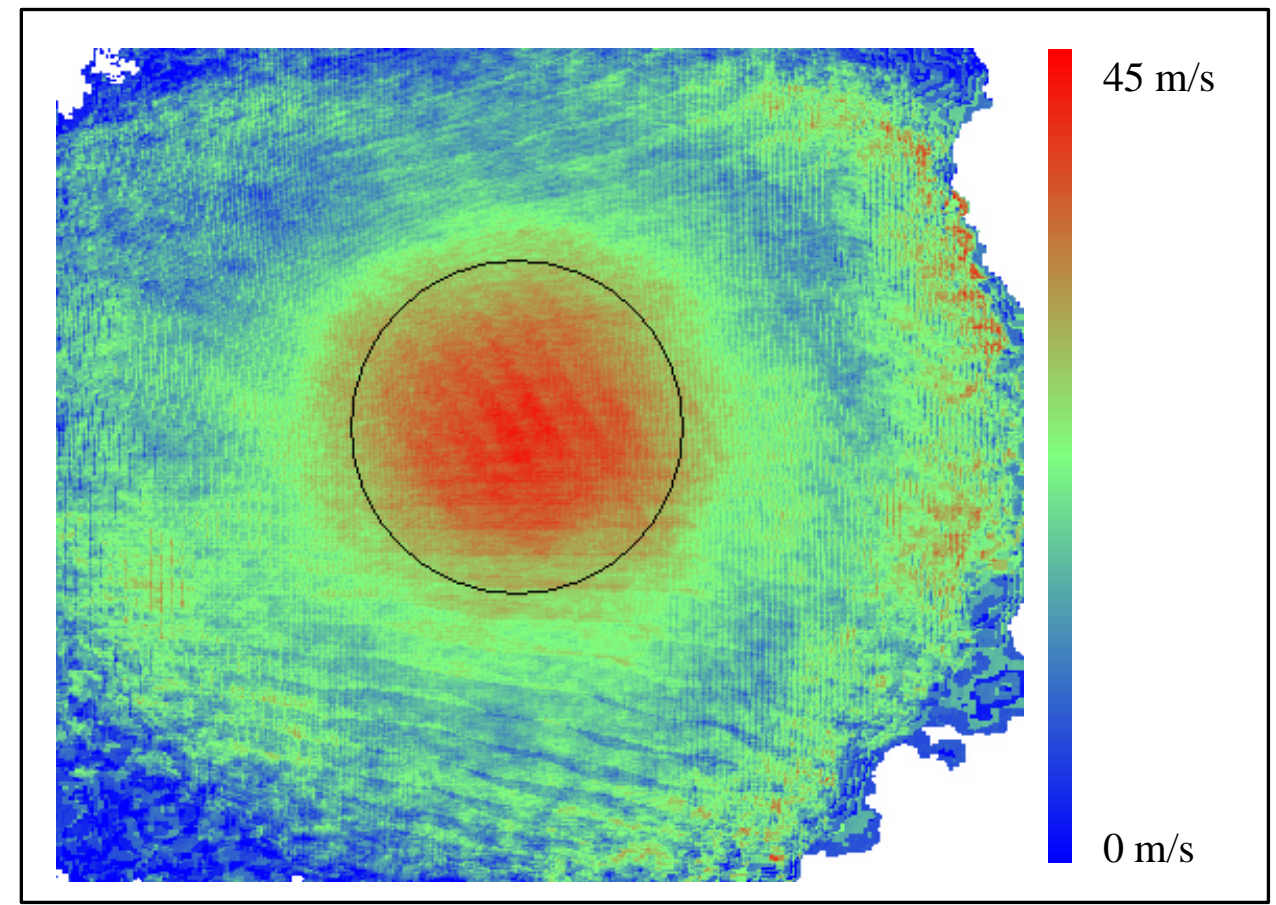

Figure 6.26. DGV velocity image of turbulent jet flow, 6 diameters from exit (Component \#2) 


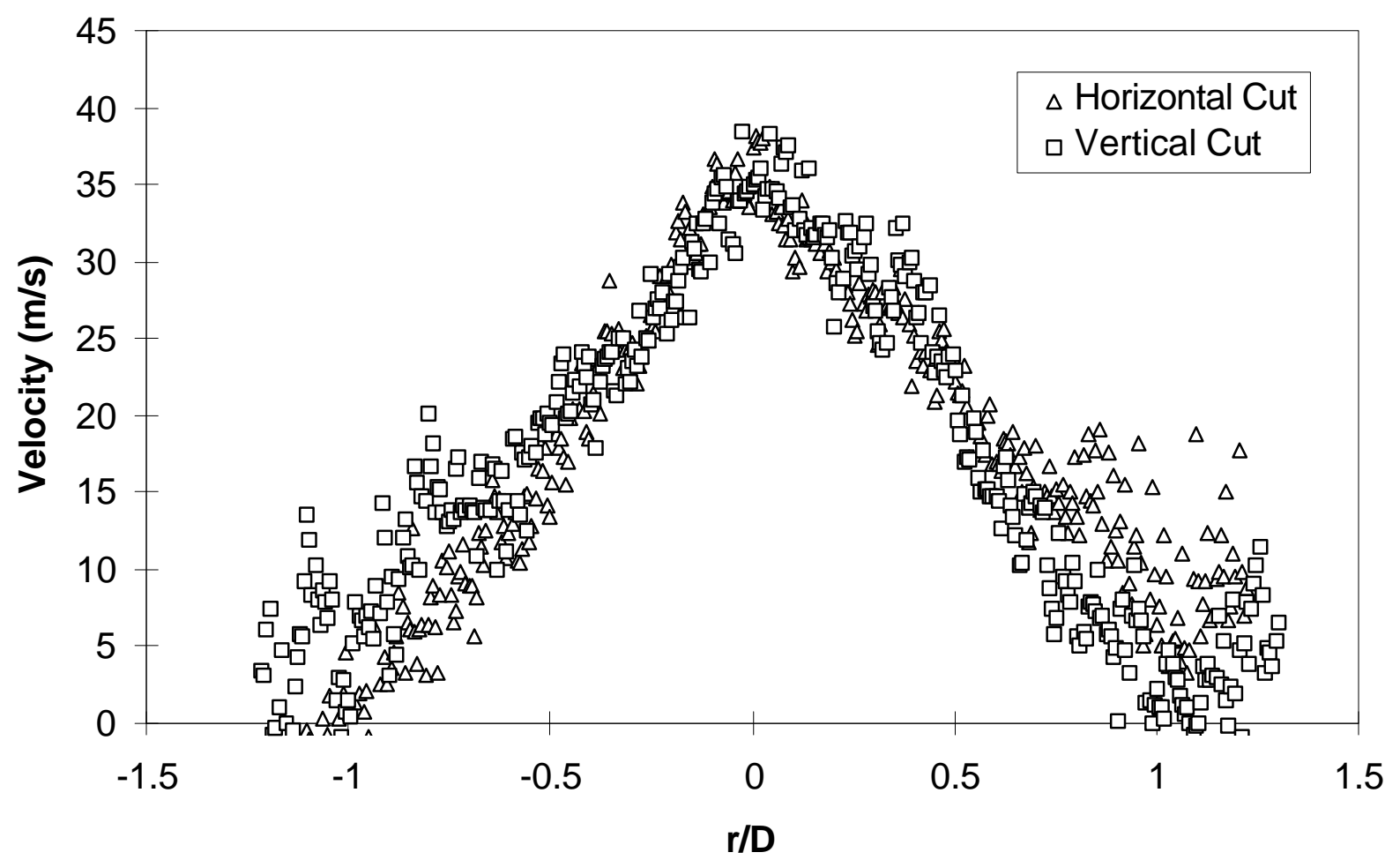

Figure 6.27. Cuts through Figure 6.25, Component \#1 DGV jet velocity measurements 6 diameters from the exit

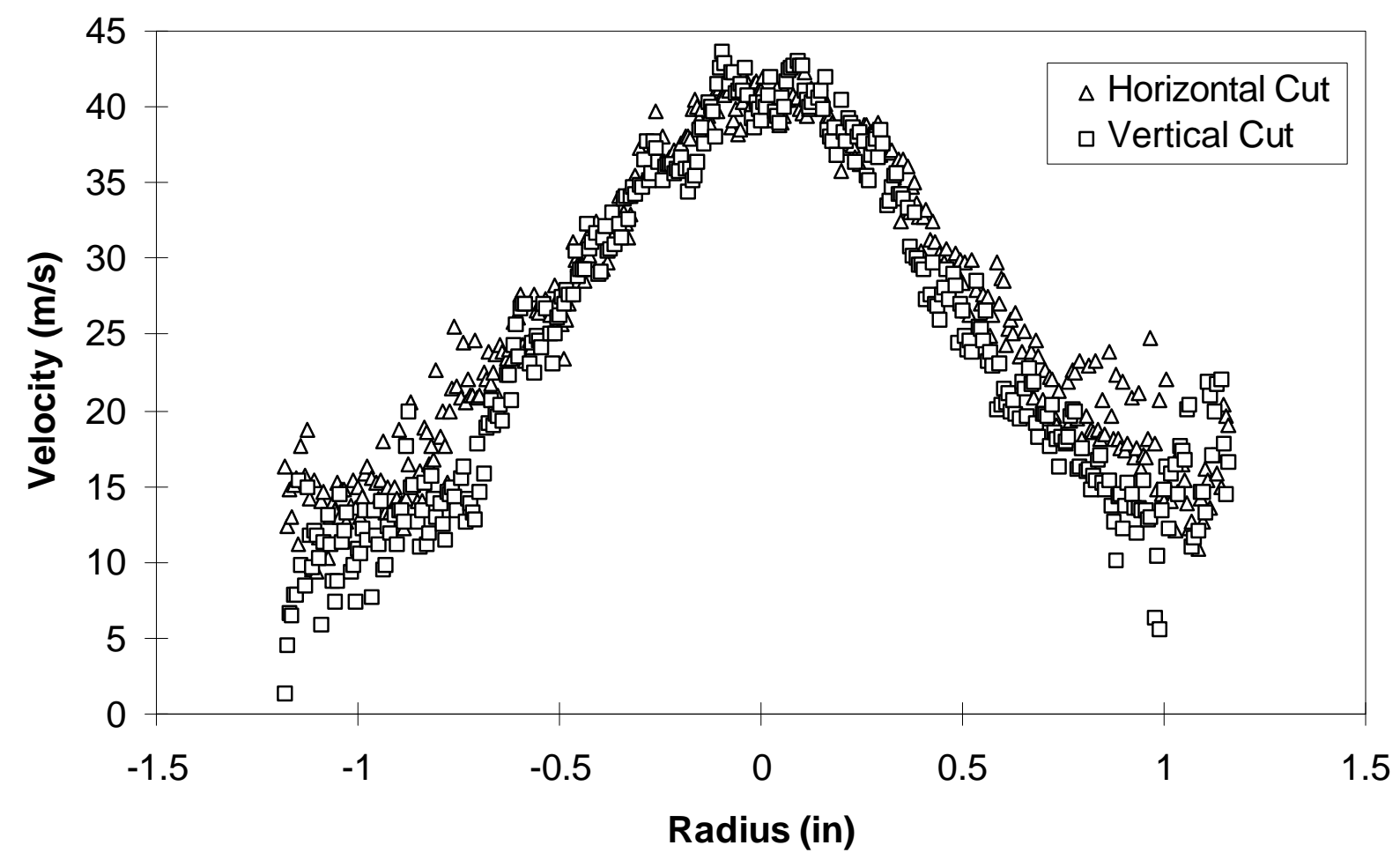

Figure 6.28. Cuts through Figure 6.26, Component \#2 DGV jet velocity measurements 6 diameters from the exit 


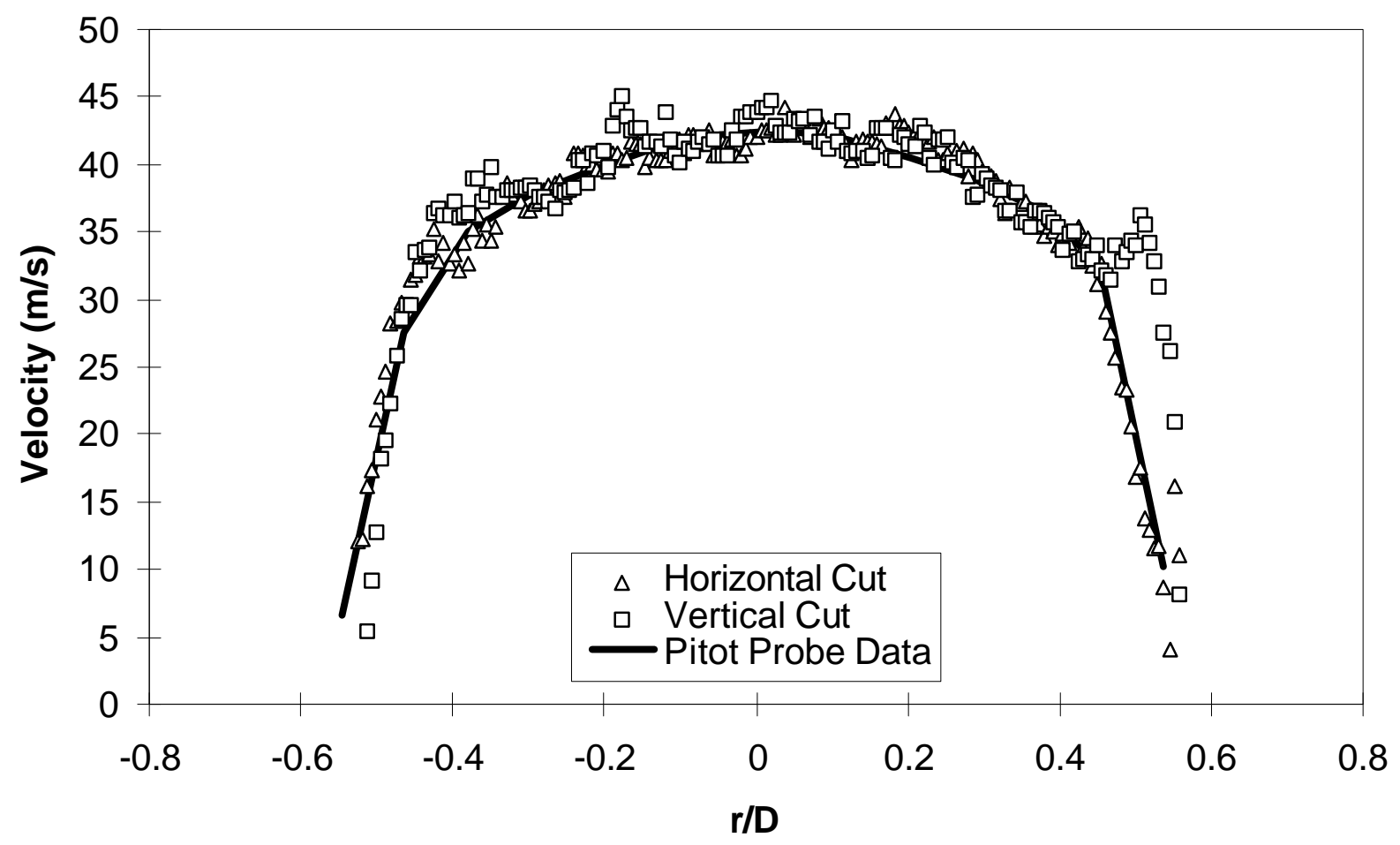

Figure 6.29. Cuts through Figure 6.11, Component \#2 DGV fully turbulent pipe flow velocity measurements with velocity offset set to match pitot probe data

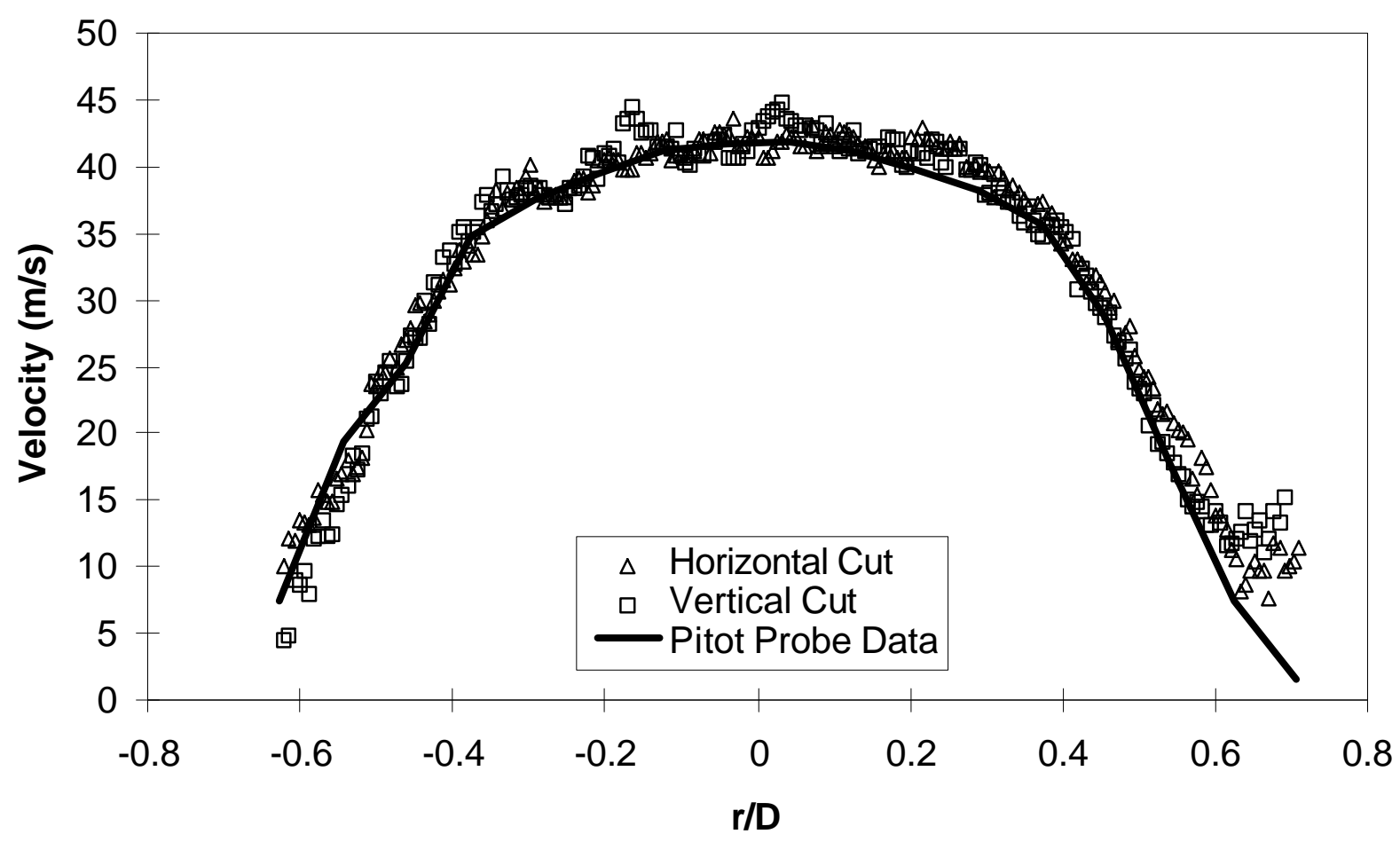

Figure 6.30. Cuts through Figure 6.12, Component \#2 DGV jet velocity measurements 1 diameter from the exit with velocity offset set to match pitot probe data 


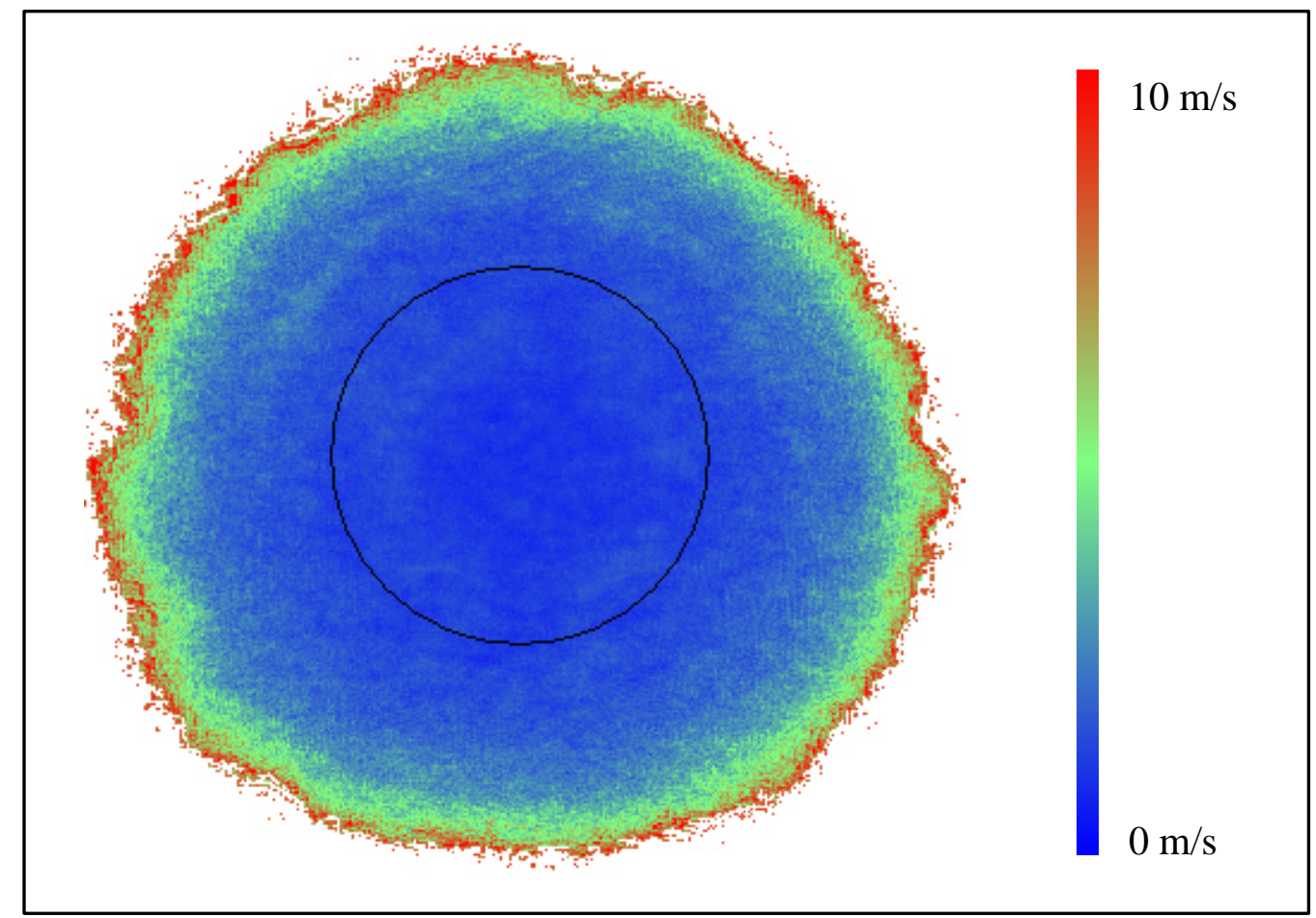

Figure 6.31. RMS fluctuations of a turbulent jet, 4 diameters from the exit (Component \#2)

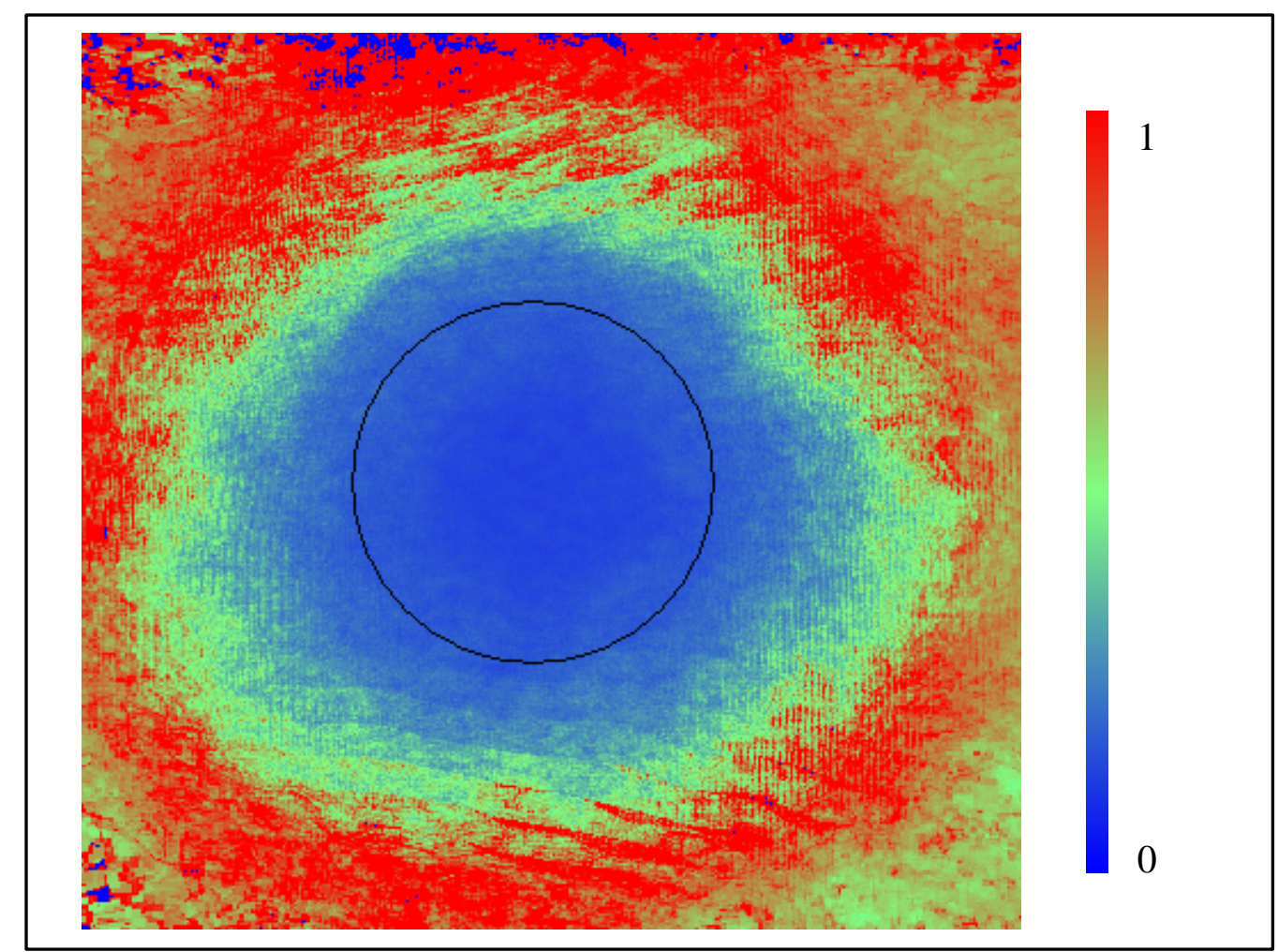

Figure 6. 32. RMS fluctuations divided by the local mean of a turbulent jet, 4 diameters from the exit (Component \#2) 


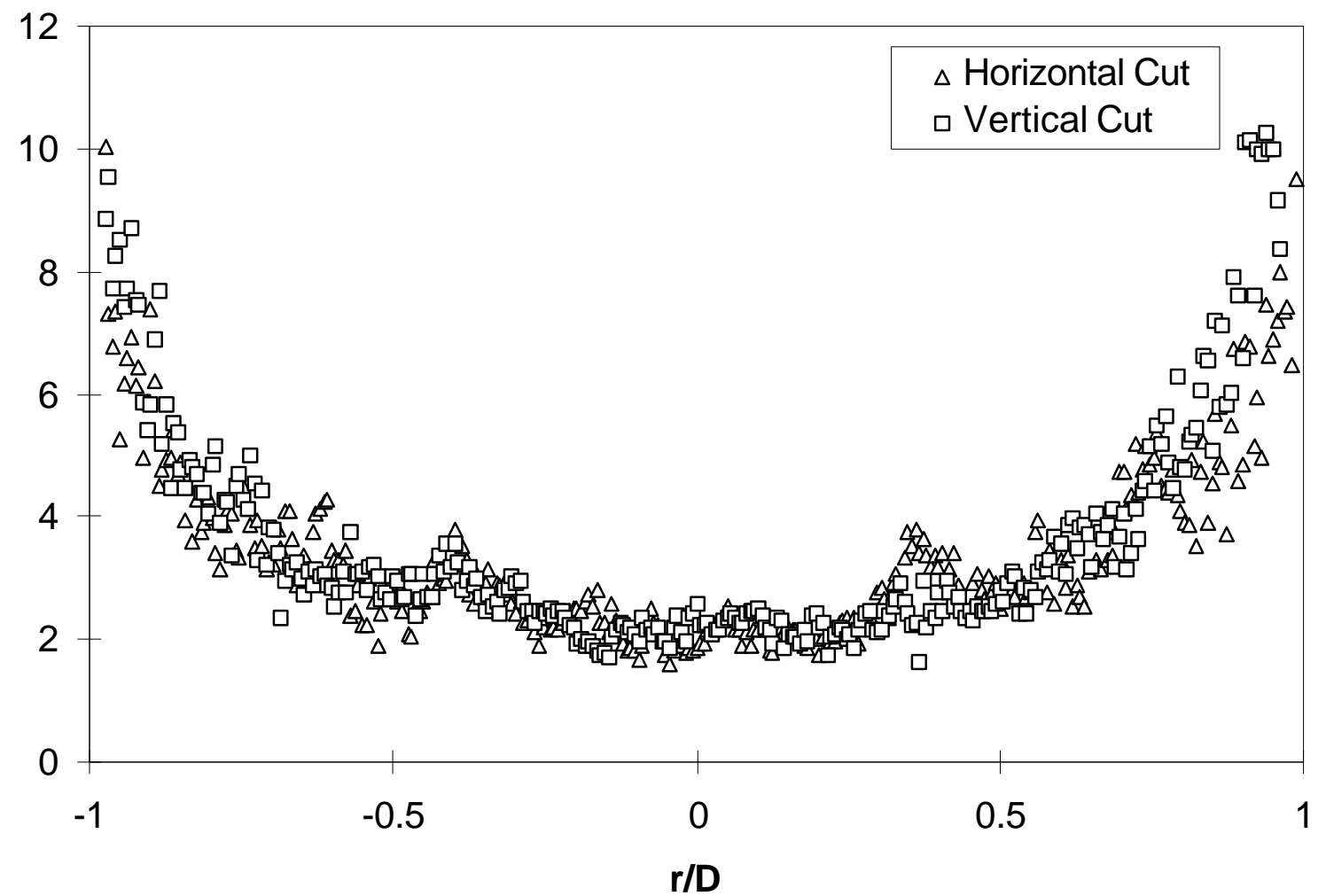

Figure 6.33. Cuts through Figure 6.25, RMS fluctuations of a turbulent jet

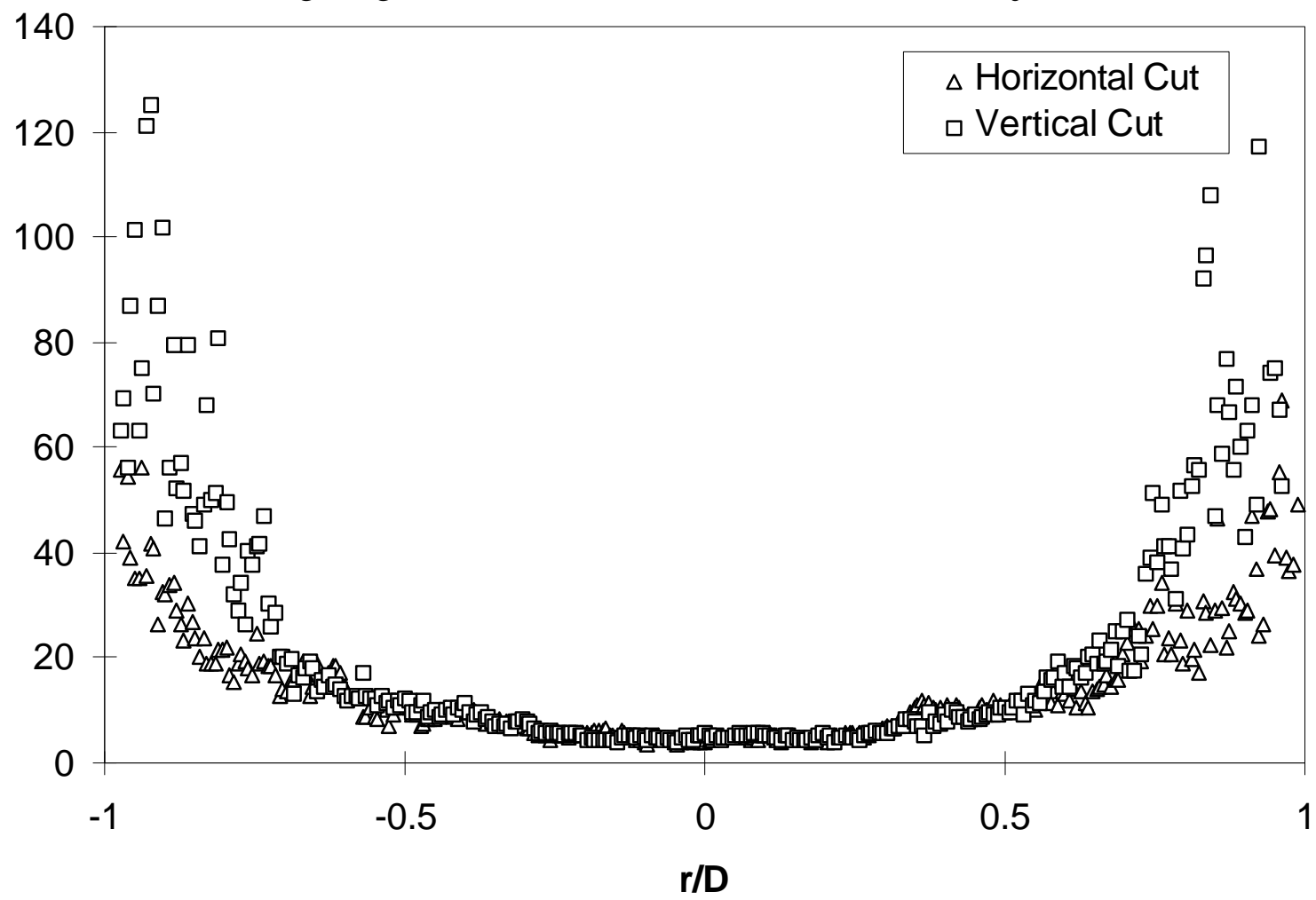

Figure 6.34. Cuts through Figure 6.26, RMS fluctuations divided by the local mean velocity 4 diameters downstream of a turbulent jet 


\section{APPENDIX A \\ Discussion of Specially Written Software}

The front ends for the image acquisition and processing routines were written in Visual Basic (VB), while the routines themselves were written in $\mathrm{C}$ and compiled into DLLs. A DLL is a piece of compiled code that contains externally callable functions. Functions written in DLLs called from VB typically execute many times faster than if that same function were written and executed in VB. This is because compiled code has been optimized and converted into machine level instructions which is readily accepted by the CPU, whereas VB code is read line by line and interpreted as it is being executed, creating serious overhead for the processor. The use of DLLs allowed a friendly user interface to be constructed in VB, while retaining the computational capabilities and advantages of compiled $\mathrm{C}$ code.

\section{Image Acquisition Software}

Before an image can be acquired, the frame grabber board must be configured to accept the signal that the camera provides as output. With different cameras having various sizes and readout rates, parameters such as voltage levels, pixel clock timings, and horizontal and vertical synchronization timings all must be configured to match the camera in use. Matrox provides a configuration utility called Intellicam to accomplish this camera interface. The utility is able to continuously grab images from a connected camera while adjustments are made to the timing and voltage parameters, so that the effect of the changes can be seen immediately. The results of the configuration utility are written to a file with a ".dcf" extension. With this utility and the flexibility of the Genesis frame grabber, a wide range of cameras can be made to smoothly interface with the board.

After the cameras were interfaced to the Genesis board, the first piece of software that was written was an image acquisition program. Image acquisition refers to the process of calling the appropriate functions provided by the frame grabber board manufacturer in order to sample images and place them into a memory buffer. The image acquisition software not only acquires images, but also provides some limited analysis information, mainly for setup and alignment purposes. There are several modes into which the software can be placed. The first mode is a single frame grab from an 
individual camera. In this mode, one of four channels is selected, and the acquired image is displayed in a separate window. While being statically displayed, the image can be analyzed in a limited fashion, as described below.

\section{Gray Level}

Gray level values can be examined at discreet pixel locations by moving the mouse cursor over the image. Every time the mouse moves over the image, calls are made to functions which enter the memory buffer with the pixel location and return the gray level value. Using the same family of functions, an array of gray level values can be returned to produce line plots displayed on the edges of the image in response to a user mouse click. Gray level inquiries are useful in detecting image saturation or approximating signal to noise ratios.

\section{False Color}

To help qualitatively discern changes in gray level value in a low contrast image, a false color routine was implemented. This routine receives a gray level image, parses the entire matrix for maximum and minimum values, then assigns colors to the range of gray level values present. It then returns an image with colors ranging from blue to red representing increasing gray level values. It is sometimes necessary to use the false color routine to see changes in gray level, since the human eye is more sensitive to changes in color then changes in brightness.

The image acquisition software can also acquire images continuously from a single camera. Continuous acquisition is mainly used for gross camera alignment and focusing. For more precise camera alignment, a mode may be selected which quickly and continuously alternates images acquired from the signal and reference cameras so that the effects of adjustments on the image overlay can be immediately seen.

\section{Multiple Cameras}

Perhaps the most directly used feature of the software is that of multiple camera acquisition. This mode allows simultaneous data grabs from multiple cameras which are synchronized at the time of acquisition. It is this simultaneous acquisition that is used for the collecting of all data and correction images. To accomplish this, a separate panel having no displayed capability is used. Options on this panel include saving each raw 
image or saving the average of all collected images, or both. In addition, there is the option to synchronize the image acquisition with the reference system photodetector voltage acquisition, which is absolutely necessary when acquiring calibration or velocity data. Synchronization is accomplished by setting two bits out of an 8 bit status word that is shared between the two acquisition systems. Bit 1 is controlled by the image acquisition system, and bit 2 by the photodetector voltage A/D board. Bit 2 is normally low until the A/D board is ready to acquire data, then it sets bit 2 to high and waits for bit 1 to go high. Bit one is low until two conditions are satisfied: the frame grabber board must be ready, and bit 2 must be high. When this happens, bit one goes high and both acquisitions start.

\section{Other Features}

The image acquisition program is able to load individual images and save any number of images in both RAW and TIF format. The program can also zoom an image from 1/16 to 16 times its original size. In addition, any time the software saves a group of

images, it automatically creates a new directory structure. The directories are created new each day with the date as the directory name, and any additional saving or reducing done on that day is placed in a numbered directory below the date. This method greatly reduces the work in finding files. Since all of the data taken on the same day is simply numbered in the date directory, a logbook listing the directories and the conditions under which the data was acquired is helpful. Future improvements to the organization of data could incorporate a text file containing test conditions written with the creation of each directory.

\section{Image Processing Software}

The image processing software performs overall data processing starting from raw correction frames and velocity data fields and ending with final velocity images. The input to the program is a listing of 8 file names, each one corresponding to a needed correction image or data file. The program has options to calculate the pixel sensitivity correction image, dewarping coefficients, and cell calibration equations, separate from the main data reduction. It also a uses a graphical interface to enter the geometry of the test configurations. With this interface, the user can click and drag vectors on the screen and 
immediately see the impact of changes to the instrument geometry. However, if angles have been previously measured, as is often the case when a test is configured, they may be entered into the program numerically. From the geometry entered, the component sensitivities are calculated for use in data reduction.

\section{Pixel Sensitivity}

Calculation of the pixel sensitivity correction image involves specifying two images to the software, and utilizing area averaging routines to find the average gray level value for each image. The area averaging routines accept an image as input, transfer each pixel value into an array, then loop through all array elements accumulating values and dividing by the number of elements. It returns a single floating point number as the average gray level value for that image. Each pixel's sensitivity is found by subtracting the gray level value for that pixel in each image and dividing by the difference in the average gray levels of the images. The process is repeated for each camera.

\section{Dewarping--Dot Finding}

Dewarping coefficients are found through a long process that starts with the selection of camera A's dot card image. The image is passed to a DLL which performs filtering on the images that identifies areas of sharp contrast, or edges. The edge finding filter is a convolution of two $3 \mathrm{X} 3$ masks with a data image. When employing masks containing these values, they are called Sobel edge finding filters. The first mask finds horizontal edges, and the second locates vertical edges. The resulting image has high values where the edges exhibit especially high contrast, and low where the image is "flat". These masks are shown below.

\begin{tabular}{|c|c|c|c|c|c|}
\hline 1 & 2 & 1 & -1 & 0 & 1 \\
\hline 0 & 0 & 0 & -2 & 0 & 2 \\
\hline-1 & -2 & -1 & -1 & 0 & 1 \\
\hline
\end{tabular}

The Sobel filtered image is then passed through a threshold function and converted into a binary image, where values above the threshold are equal to 1 , and values below the threshold are equal to 0 . This binary image is then passed to a second DLL which identifies pixel regions, having the same binary value, that are connected. These regions are identified as blobs, and they are returned back to the user overlaid with the original 
image for inspection. The user then decides on the exclusions to be passed back to the blob analysis routine. The user can click on an identified blob that should not be included or outline a grouping of extraneous blobs with the mouse. In addition, minimum and maximum blob pixel dimensions may be defined. After exclusions have been set, the image is passed back to the DLL for reprocessing. When the image is finally returned with only the correct dots identified as blobs, the remaining part of the dewarping routine may continue.

\section{Dewarping--Blob Analysis}

The center of each blob is found through a gray level weighting function that uses the gray levels of the pixels identified as blobs to find a centroid for that dot. The center coordinates are recorded as the reference grid for the warped image. The coordinates for the dewarped image are found by equally spacing the total number of dots throughout the dewarped image. This means placing all four corner dots in the corners of the image and filling in the remaining dots such that there is equal spacing between them. The dot centroids are then sorted and a transformation is calculated which will place the centers of each blob at equal intervals covering the entire image.

Before the dewarped grid is generated, the dots need to be sorted based on position because the dot finding algorithm does not necessarily return the dot positions in order, from left to right, top to bottom. The sorting algorithm takes advantage of the fact that the main source of distortion is perspective warping. This type of warping preserves vertical lines within the image, so the dots can first be sorted by their X coordinate, thus separating them into their correct columns. Next, given the number of rows in the dot card image by the user, the sorting algorithm sorts the dot list by their Y coordinate, column by column. Finally, the array is sorted to be in ascending order by rows instead of columns. This portion of the software would likely need to be rewritten for a threecomponent DGV system, where the third component would be significantly out of the horizontal plane.

\section{Dewarping--Transformation}

Once both the warped and dewarped grids are in place, the calculation of the transformation tables begins. For every pixel in the dewarped image, the four closest dots 
are found, then the relative distance away from each is calculated. These relative distances are then used to calculate the corresponding pixel position between the corresponding four dots in the warped image, using bilinear interpolation. The location of each warped pixel location is recorded in two buffers, one containing $\mathrm{X}$ coordinates, and the other containing Y coordinates for the dewarped image. This process is then repeated for each camera, but with the same dewarped grid so that corresponding dots will be in the same location for all dewarped images. The resulting files ( 2 for each camera) are essentially $\mathrm{X}$ and $\mathrm{Y}$ floating point buffer table look-ups which indicate where, in the warped image, the gray level values for that pixel in the dewarped image should come from.

\section{Cell Calibration}

The object of the cell calibration routine is to turn images taken by the cameras into relative frequency and ratio numbers that can be combined with the reference system and tabulated, so that a best-fit curve can be found. The primary mechanism for the tabulation, at least in the continuous calibration case, is the identification of mode hops.

\section{Cell Calibration--Image Averaging}

To start the calibration data reduction, the user must examine calibration images from each component to determine the best region over which the pixel gray level values should be averaged. This is necessary because the target during a calibration procedure occupies a smaller or larger space in the image for each component, based on the viewing angle. When the user selects this region, the appropriate background image is subtracted, then it is sent to an averaging routine which returns a floating point average gray level value for that particular calibration image. The images from all channels are averaged in this manner, using the same region. The result is a file that contains one column of average gray level values for each channel.

\section{Cell Calibration--Mode Transition Detection}

The next task is to identify the transition to new modes in a continuous calibration from the columns of average gray level values. When a laser operating in single frequency mode transitions from one mode to another, the power level decreases until the new mode is selected which produces a discreet change back to the original power level. 
So, to identify mode transitions, the average gray level value from one of the reference cameras is examined. When an increase in gray level from point to point is bigger than a preset value, the second point is selected as the beginning of the next mode. A graphical interface has been developed which aids in the selection of the correct threshold value for mode hop identification by displaying a plot of the average gray level values of a reference camera and highlighting which points have been identified as the start of new modes. The position of the points that begin each mode are stored and used to define the regions of ratio values that span a single mode. Any ratio points that lie between mode transitions are averaged and recorded as the representative ratio value for that mode. The result is a three column file, each column containing the ratio values for each iodine cell (Reference Cell, Component \#1, Component \#2), with one file for each calibration scan.

\section{Cell Calibration--Curve Shifting}

Several scans are used when fitting a curve through the points. The goal is to produce a more representative fit using more data points. In a single scan curve, only the spacing between modes is known; the frequency offset of each curve is arbitrary. Because of this, the curves must be shifted along the $\mathrm{X}$ axis so that the frequency offset for each curve is as close to the same as possible. The procedure for shifting requires one curve to be designated as fixed. The other curves are then slid to overlay the fixed curve by minimizing the average absolute frequency distance to the fixed curve, from each point. This produces a single scan curve shape with more points than a single scan would have.

\section{Cell Calibration--Curve Fitting}

The curve fitting software reads a file containing frequency and ratio values for the fixed and shifted scans, one pair per cell. It then plots the points for inspection, and provides the user with three choices. The first choice is a simple polynomial curve fit. When selected, the algorithm performs a least squares fit to a polynomial of a specified order. The second choice is a theory curve fit. The source of the theory curve is actually tabulated data which is stretched and shifted in both frequency and ratio directions using a Levenberg-Marquardt minimization algorithm (Press, et. al., 1992). The final choice, 
and the one that has been used most often in the present work, is a form of a Boltzmann fitting function, as shown in Eqn B2.

$$
y=\frac{A_{1}-A_{2}}{1+e^{\left(\frac{x-x_{0}}{D_{x}}+A_{2}\right)}}
$$

Here, $A_{1}$ and $A_{2}$ are the upper and lower ratio bounds of the curve, and $x_{0}$ and $D_{x}$ are frequency shifting and scaling factors, respectively. This method was used the most because its shape closely matched that of one side of an iodine absorption line, and therefore, was less likely to be affected by small variations in the shape of the calibration curve. The Boltzmann function was also fit using the Levenberg-Marquardt technique. All three curve fit methods produced small files which contained the coefficients for their respective fits so that the fits could be used in the reduction process.

\section{Velocity Calculation Software}

The last main feature of the image processing software is the routine that reads the raw data and correction images and calculates velocity images. This is the last step in producing velocity images, so all other pre-processing must be completed before running this algorithm.

The first task of the algorithm is to load into memory all correction images that are going to be applied to every data image. These include the background, white card, and pixel sensitivity images, as well as the eight dewarping coefficient buffers (2 per camera). Doing so allows improvement in speed over the case where 20 correction images are read from the disk every time a new data image is processed. The main processing loop starts by loading a raw data image. Data images are acquired as fields, so the first operation done to them is to fill in every other line with the average of the two bordering lines. Next, the appropriate background image is subtracted, then the result divided by the pixel sensitivity buffers. The next step is to low pass filter the image with a flat 5 by 5 kernel. After filtering, the images and dewarping coefficients are sent to the dewarping routine which performs bilinear interpolation on a pixel by pixel basis, as described in section 4.3.2. The result is stored and the next data image is processed. After each signal and reference image pair is reduced, the gray levels of the images are examined, and any pixel with a gray level less than 10 or equal to 255 is marked by 
replacing the gray value with a value of -1 in the reference image, and 20 in the signal image. Overwriting the data in low signal or saturated pixels with these values assures that the pixel will be identified as invalid, and will not be included in the final velocity result. The signal image is then divided by the reference image, and the result is divided by the white card correction. The white card correction is found by reading and processing the white card images before the data images, up to and including the ratio step.

After the ratio image is calculated for both velocity components, it is passed through the appropriate curve fit equations. This involves reading the coefficients from a previously stored file and processing both the reference voltage ratios and the data image ratios. The data image ratios are floating point buffers which are passed through the curve fit equations using arithmetic functions provided by the board manufacturer on an image level basis. These functions use the parallel processing features of the Genesis board to speed the calculations. As the data image ratios are being processed, any ratio values that lie outside the maximum and minimum ratio values of the calibration curve are set equal to zero. This would include any pixels which had been flagged with a -1 before forming a ratio. The curve-fit equations relate ratio to frequency, so after the image ratios are processed, the buffers contain measured frequency, from which the reference frequency is subtracted. This gives a Doppler frequency measurement in the direction of the bisector of the angle between the laser sheet and the viewing angle of the measurement system. The velocity is calculated from the Doppler frequency measurement by using Equation 1, which contains geometry parameters and the laser wavelength. At this point, the velocity vectors are in directions defined by the system geometry. It is more desirable, however, to express the velocity of a object or flow in an orthogonal coordinate system which is aligned with some physical aspect of the flow or the setup. Therefore, a transformation, shown in Appendix A, was implemented which converts the velocity vectors to their orthogonal components, perpendicular and parallel to the laser sheet.

\section{Image Calculator}

Another often used piece of software is an analysis program that was written to behave somewhat like a calculator. The goal was to make single operations available to 
examine a specific image or set of images in more detail. This is useful for debugging, as well as in checking data validity. For example, the buttons on the main panel perform discrete operations on images such as subtract, low pass filter, multiply, divide, average, interlace, and make cuts. Each of these functions, with the exception of making cuts, is found in the image acquisition/processing programs, but exists only as part of a bigger operation. In the image calculator, these functions have been extracted and made available individually. The function which makes cuts is derived from the image acquisition function which plotted $\mathrm{X}$ and $\mathrm{Y}$ cuts through an acquired image. The procedure was automated to process many runs of velocity images at once, naming and storing them appropriately.

\section{Photodetector Acquisition}

The photodetector acquisition program was originally written when the DGV system existed only as a point based system with photodetectors in place of cameras. Then, all acquisition was handled with the photodetector acquisition program, and it was flexible enough to accommodate single or multiple velocity channel acquisition. It was modified for the image based system mainly by changing the way data was acquired. Instead of handling all timing of acquisition internally, there needed to be a way to synchronize the acquisition of data from the photodetectors with that from the cameras. Synchronization was accomplished via a hardware trigger and the setting of status bits as described in the image acquisition program section.

The main function of the photodetector acquisition program is to sample the photodetector voltages from multiple channels simultaneously and plot them for examination. The main panel has many user configurable options such as sampling rate, number of samples, voltage range, and channel selection. It has the ability to save standard deviations, voltage ratios, average values, and raw voltages to a data file. It also has options concerning the status of the current trigger state. At each acquisition, the software plots a voltage trace for two channels as well as a ratio trace. The ratio trace is useful in quickly determining the laser setting and it's position down the side of the absorption band. It is also used during calibrations to observe the quality of the scan. 


\section{APPENDIX B}

\section{Development of Orthogonal Velocity Equations}

The constraints on the geometry of the two-component DGV measuring system may be such that the measurement vectors cannot be aligned perpendicular to each other. In general, a transformation is needed to extract two-component, orthogonal velocity vectors aligned in physically meaningful directions. The transformation is accomplished using the Doppler frequency equation (Equation 1):

$\Delta f=\frac{f_{\mathrm{o}}}{\mathrm{c}}(\hat{\mathrm{a}}-\hat{\mathrm{l}}) \cdot \overrightarrow{\mathrm{V}}$

where $f$ is frequency, $\mathrm{c}$ is the speed of light, a and 1 are unit vectors in the direction of the viewing and laser directions, respectively. The laser frequency and speed of light can be combined to define the wavelength. The wavelength used in this research was $514.5 \mathrm{~nm}$.

$$
\frac{f_{\mathrm{o}}}{\mathrm{c}}=\lambda_{\mathrm{o}}
$$

Substituting Equation B2 into B1, and expanding into vector components, yields, for the first component:

$\Delta f_{1}=\left(\cos \theta_{1} \hat{i}+\sin \theta_{1} \hat{j}+\cos \gamma_{L} \hat{i}+\sin \gamma_{L} \hat{j}\right) \bullet \frac{(U \hat{i}+V \hat{j})}{\lambda_{o}}$

The angle between the designated $\mathrm{x}$-axis and the viewing direction of the first component is given by $\theta_{1}$, and $\gamma_{\mathrm{L}}$ is the angle that the laser makes with the $\mathrm{x}$-axis.

Simplifying,

$$
\begin{aligned}
& \lambda_{\mathrm{o}} \Delta f_{1}=\mathrm{U}\left(\cos \theta_{1}+\cos \gamma_{\mathrm{L}}\right) \hat{\mathrm{i}}+\mathrm{V}\left(\sin \theta_{1}+\sin \gamma_{\mathrm{L}}\right) \hat{\mathrm{j}} \\
& \mathrm{C}_{1}=\cos \theta_{1}+\cos \gamma_{\mathrm{L}} \quad \mathrm{S}_{1}=\sin \theta_{1}+\sin \gamma_{\mathrm{L}}
\end{aligned}
$$

$\lambda_{\mathrm{o}} \Delta f_{1}=\mathrm{C}_{1} \mathrm{U} \hat{\mathrm{i}}+\mathrm{S}_{1} \mathrm{~V} \hat{\mathrm{j}}$

Repeating for the second component:

$$
\Delta f_{2}=\left(\cos \theta_{2} \hat{i}+\sin \theta_{2} \hat{j}+\cos \gamma_{L} \hat{i}+\sin \gamma_{L} \hat{j}\right) \bullet \frac{(U \hat{i}+V \hat{j})}{\lambda_{o}}
$$




$$
\begin{array}{ll}
\lambda_{\mathrm{o}} \Delta f_{2} & =\mathrm{U}\left(\cos \theta_{2}+\cos \gamma_{\mathrm{L}}\right) \hat{\mathrm{i}}+\mathrm{V}\left(\sin \theta_{2}+\sin \gamma_{\mathrm{L}}\right) \hat{\mathrm{j}} \\
\mathrm{C}_{2}=\cos \theta_{2}+\cos \gamma_{\mathrm{L}} \quad \mathrm{S}_{2}=\sin \theta_{2}+\sin \gamma_{\mathrm{L}} & \\
\lambda_{\mathrm{o}} \Delta f_{2} & =\mathrm{C}_{2} \mathrm{U} \hat{\mathrm{i}}+\mathrm{S}_{2} \mathrm{~V} \hat{\mathrm{j}}
\end{array}
$$

Solving for $\mathrm{U}$ and $\mathrm{V}$ by combining Equations B6-1 and B6-2

Multiplying B6-1 by $\mathrm{S}_{2}$ :

$\mathrm{S}_{2}\left(\lambda_{\mathrm{o}} \Delta f_{1}=\mathrm{C}_{1} \mathrm{U}_{\hat{\mathrm{i}}}+\mathrm{S}_{1} \mathrm{~V}_{\hat{\mathrm{j}}}\right) \Rightarrow \lambda_{\mathrm{o}} \Delta f_{1} \mathrm{~S}_{2}=\mathrm{C}_{1} \mathrm{~S}_{2} \mathrm{U}_{\hat{\mathrm{i}}}+\mathrm{S}_{1} \mathrm{~S}_{2} \mathrm{~V}_{\hat{\mathrm{j}}}$

Multiplying B6-2 by $\mathrm{S}_{1}$ :

$\mathrm{S}_{1}\left(\lambda_{\mathrm{o}} \Delta f_{2}=\mathrm{C}_{2} \mathrm{U}_{\hat{\mathrm{i}}}+\mathrm{S}_{2} \mathrm{~V}_{\hat{\mathrm{j}}}\right) \Rightarrow \lambda_{\mathrm{o}} \Delta f_{2} \mathrm{~S}_{1}=\mathrm{C}_{2} \mathrm{~S}_{1} \mathrm{U}_{\hat{\mathrm{i}}}+\mathrm{S}_{1} \mathrm{~S}_{2} \mathrm{~V}_{\hat{\mathrm{j}}}$

Subtracting B8 from B7:

$\lambda_{\mathrm{o}}\left(\Delta f_{1} \mathrm{~S}_{2}-\Delta f_{2} \mathrm{~S}_{1}\right)=\mathrm{C}_{1} \mathrm{~S}_{2} \mathrm{U}_{\hat{\mathrm{i}}}-\mathrm{C}_{2} \mathrm{~S}_{1} \mathrm{U}_{\hat{\mathrm{i}}}=\mathrm{U}_{\hat{\mathrm{i}}}\left(\mathrm{C}_{1} \mathrm{~S}_{2}-\mathrm{C}_{2} \mathrm{~S}_{1}\right)$

Similarly,

$\lambda_{\mathrm{o}}\left(\Delta f_{1} \mathrm{C}_{2}-\Delta f_{2} \mathrm{C}_{1}\right)=\mathrm{C}_{2} \mathrm{~S}_{1} \mathrm{~V}-\mathrm{C}_{1} \mathrm{~S}_{2} \mathrm{~V}=\mathrm{V}\left(\mathrm{C}_{2} \mathrm{~S}_{1}-\mathrm{C}_{1} \mathrm{~S}_{2}\right)$

Solving for U from Equation B9:

$$
\mathrm{U} \hat{\mathrm{i}}=\lambda_{\mathrm{o}} \frac{\Delta f_{1} \mathrm{~S}_{2}-\Delta f_{2} \mathrm{~S}_{1}}{\mathrm{C}_{1} \mathrm{~S}_{2}-\mathrm{C}_{2} \mathrm{~S}_{1}}
$$

Solving for V from Equation B10:

$$
\mathrm{V} \hat{\mathrm{j}}=\lambda_{\mathrm{o}} \frac{\Delta f_{1} \mathrm{C}_{2}-\Delta f_{2} \mathrm{C}_{1}}{\mathrm{C}_{2} \mathrm{~S}_{1}-\mathrm{C}_{1} \mathrm{~S}_{2}}
$$

Equations B11 and B12 were used in the orthogonal data reduction process. 


\title{
Development and Accuracy Determination of a Two-Component Doppler Global Velocimeter (DGV)
}

By

\author{
Stephen M. Naylor \\ DISSERTATION
}

Submitted to the College of Engineering and Mineral Resources

West Virginia University

Morgantown, WV

APPROVAL OF THE EXAMINING COMMITTEE

Larry E. Banta, Ph.D.

Gary J. Morris, Ph.D.

Charles F. Stanley, Ph.D.

Date

John M. Kuhlman, Ph.D., Chair 